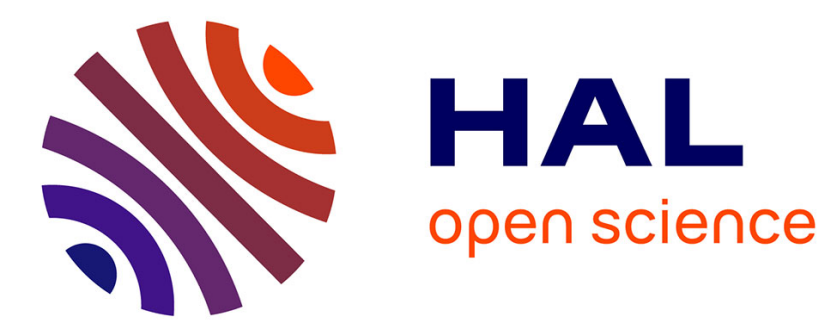

\title{
Minimal Bubbling for Willmore Surfaces
}

Nicolas Marque

\section{To cite this version:}

Nicolas Marque. Minimal Bubbling for Willmore Surfaces. International Mathematics Research Notices, 2020, 0000, 10.1093/imrn/rnaa079 . hal-02985290

\section{HAL Id: hal-02985290 \\ https://hal.science/hal-02985290}

Submitted on 2 Nov 2020

HAL is a multi-disciplinary open access archive for the deposit and dissemination of scientific research documents, whether they are published or not. The documents may come from teaching and research institutions in France or abroad, or from public or private research centers.
L'archive ouverte pluridisciplinaire HAL, est destinée au dépôt et à la diffusion de documents scientifiques de niveau recherche, publiés ou non, émanant des établissements d'enseignement et de recherche français ou étrangers, des laboratoires publics ou privés. 


\title{
Minimal bubbling for Willmore surfaces.
}

\author{
Nicolas Marque ${ }^{1}$ \\ ${ }^{1}$ Institut Mathématique de Jussieu, Paris VII, Bâtiment Sophie Germain, Case 7052, 75205 Paris \\ Cedex 13, France
}

Correspondence to be sent to: nicolas.marque@imj-prg.fr

\begin{abstract}
In this paper we build an explicit example of a minimal bubble on a Willmore surface, showing there cannot be compactness for Willmore immersions of Willmore energy above $16 \pi$. Additionally we prove an inequality on the second residue for limits sequences of Willmore immersions with simple minimal bubbles. Doing so, we exclude some gluing configurations and prove compactness for immersed Willmore tori of energy below $12 \pi$.
\end{abstract}

\section{Contents}

\begin{tabular}{lll}
\hline 1 & Introduction & 1
\end{tabular}

\begin{tabular}{|ll|l|l|l|}
\hline 2 & Proof of theorem $\mid 1.3$ & 6
\end{tabular}

\begin{tabular}{|ll|l|l|l|}
\hline & Setting for a proof of theorem & 1.5 & in local conformal charts: & 10
\end{tabular}

3.1 Convergence in local conformal charts: f . . . . . . . . . . . . . . . . . . . . 10

$3.2 \quad$ Branch point-branched end correspondence: . . . . . . . . . . . . . . . . . . . . . . . . 13

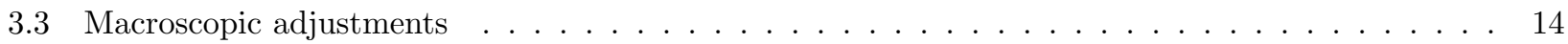

3.4 Infinitesimal adjustments: . . . . . . . . . . . . . . . . . . . . . . . . . . . . . 16

\begin{tabular}{|ll}
\hline 4 & Expanding the conformal factor
\end{tabular}

5 Conditions on the limit surface: 2

5.1 Proof of theorem $\mid 1.5 \square \ldots \ldots \ldots \ldots \ldots \ldots \ldots \ldots$

5.2 Proof of corollary 1.6$] \ldots \ldots \ldots \ldots \ldots \ldots \ldots \ldots$

5.3 An exploration of the consequences of theorem $1.5 \ldots \ldots \ldots$

\begin{tabular}{|l|}
\hline A Appendix \\
\hline
\end{tabular}

\begin{tabular}{|l}
\hline Bibliography \\
\hline
\end{tabular}

\section{Introduction}

The following is primarily concerned with the study of Willmore immersions in $\mathbb{R}^{3}$. Let $\vec{\Phi}$ be an immersion from a closed Riemann surface $\Sigma$ into $\mathbb{R}^{3}$. We denote by $g:=\vec{\Phi}^{*} \xi$ the pullback by $\vec{\Phi}$ of the Euclidean metric $\xi$ of $\mathbb{R}^{3}$, also called the first fundamental form of $\vec{\Phi}$ or the induced metric. Let $d \mathrm{vol}_{g}$ be the volume form associated with $g$. The Gauss map $\vec{n}$ of $\vec{\Phi}$ is the normal to the surface. In local coordinates $(x, y)$ :

$$
\vec{n}:=\frac{\vec{\Phi}_{x} \times \vec{\Phi}_{y}}{\left|\vec{\Phi}_{x} \times \vec{\Phi}_{y}\right|},
$$

Received 1 Month 20XX; Revised 11 Month 20XX; Accepted 21 Month 20XX

Communicated by A. Editor

(C) The Author 0000. Published by Oxford University Press. All rights reserved. For permissions,

please e-mail: journals.permissions@oxfordjournals.org. 
where $\vec{\Phi}_{x}=\partial_{x} \vec{\Phi}, \vec{\Phi}_{y}=\partial_{y} \vec{\Phi}$ and $\times$ is the usual cross product in $\mathbb{R}^{3}$. Denoting $\pi_{\vec{n}}$ the orthonormal projection on the normal (meaning $\pi_{\vec{n}}(v)=\langle\vec{n}, v\rangle \vec{n}$ ), the second fundamental form of $\vec{\Phi}$ at the point $p \in \Sigma$ is defined as follows.

$$
\vec{A}_{p}(X, Y):=A_{p}(X, Y) \vec{n}:=\pi_{\vec{n}}\left(d^{2} \vec{\Phi}(X, Y)\right) \text { for all } X, Y \in T_{p} \Sigma .
$$

The mean curvature of the immersion at $p$ is then

$$
\vec{H}(p)=H(p) \vec{n}=\frac{1}{2} \operatorname{Tr}_{g}(A) \vec{n}
$$

while its tracefree second fundamental form is

$$
\AA_{p}(X, Y)=A_{p}(X, Y)-\frac{1}{2} H(p) g_{p}(X, Y) .
$$

The Willmore energy is defined as

$$
W(\vec{\Phi}):=\int_{\Sigma} H^{2} d \operatorname{vol}_{g} .
$$

Willmore immersions are critical points of this Willmore energy, and satisfy the Willmore equation:

$$
\Delta_{g} H+|\AA|^{2} H=0 \text {. }
$$

The Willmore energy was already under scrutiny in the XIXth century in the study of elastic plates, but to our knowledge W. Blaschke was the first to state (see [5]) its invariance by conformal diffeomorphisms of $\mathbb{R}^{3}$ (which was later rediscovered by T. Willmore, see [25]) and to study it in the context of conformal geometry.

While the Willmore energy is the canonically studied Lagrangian, and serves as a natural measure of the complexity of a given immersion, its invariance is contextual. Indeed $W$ is not invariant by inversions whose center is on the surface, with the simplest example being the Euclidean sphere which is sent to a plane once inverted at one of its points. The true pointwise conformal invariant (as shown by T. Willmore, 25]) is in fact $\left|\AA_{p}\right| d \operatorname{vol}_{g_{p}}$. The total curvature and tracefree curvature are then two relevant energies, respectively defined as follows:

$$
\begin{aligned}
& E(\vec{\Phi}):=\int_{\Sigma}|A|_{g}^{2} d \operatorname{vol}_{g}=\int_{\Sigma}\left|\nabla_{g} \vec{n}\right|^{2} d \operatorname{vol}_{g} \\
& \mathcal{E}(\vec{\Phi}):=\int_{\Sigma}|\AA|_{g}^{2} d \operatorname{vol}_{g} .
\end{aligned}
$$

Quick and straightforward computations (done for instance in appendix A.1 of [19] in a conformal chart) ensure that both

$$
E(\vec{\Phi})=4 W(\vec{\Phi})-4 \pi \chi(\Sigma)
$$

with $\chi(\Sigma)$ the Euler characteristic of $\Sigma$, and

$$
\mathcal{E}(\vec{\Phi})=2 W(\vec{\Phi})-4 \pi \chi(\Sigma)
$$

The conformal invariance of $W$ when the topology of the surface is not changed then follows from (3). A Willmore surface is thus a critical point of $W, E$ and $\mathcal{E}$.

In the study of the moduli spaces of Willmore immersions, the compactness question has proven pivotal. E. Kuwert and R. Schätzle (see [9]) and later T. Rivière (in arbitrary codimension see for instance theorem I.5 in [24) showed that Willmore immersions follow an $\varepsilon$-regularity result. These induce a now classical concentration of compactness dialectic, as originally developed by J. Sacks and K. Uhlenbeck, for Willmore surfaces with bounded total curvature (or alternatively, given (2), bounded Willmore energy and topology). In essence, sequences of Willmore surfaces converge smoothly away from concentration points, on which trees of Willmore spheres are blown (see [6] for an exploration of the bubble tree phenomenon in another simpler case). Y. Bernard and T. Rivière developed an energy quantization result for such sequences of Willmore immersions assuming their conformal class is in a compact of the Teichmuller space (see theorem I.2 in 4]). P. Laurain and T. Rivière then showed one could replace the bounded conformal class hypothesis by a weaker convergence of residues linked with the conservation laws. Since we will work with bounded conformal classes we here give abridged versions of theorems I.2 and I.3 of [4]. 
Theorem 1.1. Let $\vec{\Phi}_{k}$ be a sequence of Willmore immersions of a closed surface $\Sigma$. Assume that

$$
\limsup _{k \rightarrow \infty} W\left(\vec{\Phi}_{k}\right)<\infty
$$

and that the conformal class of $\vec{\Phi}_{k}^{*} \xi$ remains within a compact subdomain of the moduli space of $\Sigma$. Then modulo extraction of a subsequence, the following energy identity holds

$$
\lim _{k \rightarrow \infty} W\left(\vec{\Phi}_{k}\right)=W\left(\vec{\Phi}_{\infty}\right)+\sum_{s=1}^{p} W\left(\vec{\eta}_{s}\right)+\sum_{t=1}^{q}\left[W\left(\vec{\zeta}_{t}\right)-4 \pi \theta_{t}\right]
$$

where $\vec{\Phi}_{\infty}$ (respectively $\vec{\eta}_{s}, \vec{\zeta}_{t}$ ) is a possibly branched smooth immersion of $\Sigma$ (respectively $\mathbb{S}^{2}$ ) and $\theta_{t} \in \mathbb{N}$.

Further there exists $a^{1} \ldots a^{n} \in \Sigma$ such that

$$
\vec{\Phi}_{k} \rightarrow \vec{\Phi}_{\infty} \text { in } C_{\mathrm{loc}}^{\infty}\left(\Sigma \backslash\left\{a^{1}, \ldots, a^{n}\right\}\right)
$$

up to conformal diffeomorphisms of $\mathbb{R}^{3} \cup\{\infty\}$. Moreover there exists a sequence of radii $\rho_{k}^{s}$, points $x_{k}^{s} \in \mathbb{C}$ converging to one of the $a^{i}$

such that up to conformal diffeomorphisms of $\mathbb{R}^{3}$

$$
\vec{\Phi}_{k}\left(\rho_{k}^{s} y+x_{k}^{s}\right) \rightarrow \vec{\eta}_{s} \circ \pi^{-1}(y) \text { in } C_{\text {loc }}^{\infty}(\mathbb{C} \backslash\{\text { finite set }\})
$$

Finally there exists a sequence of radii $\rho_{k}^{t}$, points $x_{k}^{t} \in \mathbb{C}$ converging to one of the $a^{i}$ such that up to conformal diffeomorphisms of $\mathbb{R}^{3}$

$$
\vec{\Phi}_{k}\left(\rho_{k}^{t} y+x_{k}^{t}\right) \rightarrow \iota_{p_{t}} \circ \vec{\zeta}_{t} \circ \pi^{-1}(y) \text { in } C_{\text {loc }}^{\infty}(\mathbb{C} \backslash\{\text { finite set }\}) .
$$

Here $\iota_{p_{t}}$ is an inversion at $p \in \zeta_{t}\left(\mathbb{S}^{2}\right)$. The integer $\theta_{t}$ is the density of $\zeta_{t}$ at $p_{t}$.

While theorem 1.1 states an energy quantization for $W$, equality VIII.8 in [4] offers in fact a stronger energy quantization for $E$ (and one for $\mathcal{E}$ follows). The $a^{i}$ are the aforementioned concentration points and the $\vec{\eta}_{s}$ and $\iota_{p_{t}} \circ \vec{\zeta}_{t}$ are the bubbles blown on those concentration points. More precisely, the $\vec{\eta}_{s}$ are the compact bubbles, while the $\iota_{p_{t}} \circ \vec{\zeta}_{t}$ are the non compact ones. Non-compact bubbles stand out as a consequence of the conformal invariance of the problem (see [11 to compare with the bubble tree extraction in the constant mean curvature framework). One might notice that $W\left(\iota_{p_{t}} \circ \vec{\zeta}_{t}\right)=W\left(\vec{\zeta}_{t}\right)-4 \pi \theta_{t}$, and deduce that if $W\left(\vec{\zeta}_{t}\right)=4 \pi \theta_{t}$, then the bubble $\iota_{p_{t}} \circ \vec{\zeta}_{t}$ is minimal. This case, which we will refer to as minimal bubbling will be of special interest to us in this article. Furthermore, if there is only one bubble at a given concentration point we will call the bubbling simple.

Works from Y. Li in [15] (see also [12]) ensure that compact simple bubbles cannot appear. These studies, furthered by P. Laurain and T. Rivière (see theorem 0.2 of [12, written just below) have yielded a compactness result for Willmore immersions of energy strictly below $12 \pi$.

Theorem 1.2. Let $\Sigma$ be a closed surface of genus $g \geq 1$ and $\vec{\Phi}_{k}: \Sigma \rightarrow \mathbb{R}^{3}$ a sequence of Willmore immersions such that the induced metric remains in a compact set of the moduli space and

$$
\limsup _{k \rightarrow \infty} W\left(\vec{\Phi}_{k}\right)<12 \pi
$$

Then there exists a diffeomorphism $\psi_{k}$ of $\Sigma$ and a conformal transformation $\Theta_{k}$ of $\mathbb{R}^{3} \cup\{\infty\}$, such that $\Theta_{k} \circ \vec{\Phi}_{k} \circ \psi_{k}$ converges up to a subsequence toward a smooth Willmore immersion $\vec{\Phi}_{\infty}: \Sigma \rightarrow \mathbb{R}^{3}$ in $C^{\infty}(\Sigma)$.

In the aforementioned paper P. Laurain and T. Rivière put forth a potential candidate for Willmore bubbling, consisting of an Enneper bubble glued on the branch point of an inverted Chen-Gackstatter torus, with an energy of exactly $12 \pi$ (see [8] for the definition of the Chen-Gackstatter torus).

However before considering genus one sequences, a study of the spherical case offers interesting perspectives. Indeed in his seminal work [7], R. Bryant offered a classification of Willmore immersions of a sphere in $\mathbb{R}^{3}$, showed they were conformal transforms of minimal immersions (see theorem $\mathrm{F}$ ), and thus that their Willmore energy was $4 \pi$-quantized. Moreover while giving a complete description of the Willmore immersions of energy $16 \pi$ (part 5), R. Bryant remarked: 
"Surprisingly, this space [of Willmore immersions of energy $16 \pi$ ] is not compact."

It is then interesting to consider whether one can degenerate a sequence of $16 \pi$ immersions into a bubble blown on a Willmore sphere. A quick study direct our search toward the most likely case: a sequence degenerating into an Enneper immersion glued on the branch point of the inverse of a López minimal surface (that is a minimal sphere with one branched end of multiplicity 3 and one end of multiplicity 1 , see (10) below for a parametrization). This will be our first result:

Theorem 1.3. There exists $\vec{\Phi}_{k}: \mathbb{S}^{2} \rightarrow \mathbb{R}^{3}$ a sequence of Willmore immersions such that

$$
W\left(\vec{\Phi}_{k}\right)=16 \pi
$$

and

$$
\vec{\Phi}_{k} \rightarrow \vec{\Phi}_{\infty}
$$

smoothly on $\mathbb{S}^{2} \backslash\{0\}$, where $\vec{\Phi}_{\infty}$ is the inversion of a López surface. Moreover,

$$
\lim _{k \rightarrow \infty} E\left(\vec{\Phi}_{k}\right)=E\left(\vec{\Phi}_{\infty}\right)+E\left(\Psi_{\infty}\right)
$$

where $\Psi_{\infty}: \mathbb{C} \rightarrow \mathbb{R}^{3}$ is the immersion of an Enneper surface.

Theorem 1.3 proves that minimal bubbles can appear and thus that Willmore immersions are not compact. It might also indicate the possibility of gluing an Enneper bubble on an inverted Chen-Gackstatter torus. However R. Bryant's classification result proves that one cannot glue an Enneper bubble on an inverted Enneper surface (the resulting surface would be of energy $12 \pi$, and thus limit of Willmore immersions of equal energy, which R. Bryant showed did not exist). The local behavior of the limit surface around its branch point needs then to be constrained in order to forbid this case. Since the Chen-Gackstatter torus and the Enneper surface are asymptotic near their branched end there is hope yet to eliminate this configuration.

The behavior of a Willmore surface around a branch point can be fully described by an expansion, proven by Y. Bernard and T. Rivière in theorem 1.8 of [3].

Theorem 1.4. Let $\vec{\Phi} \in C^{\infty}(\mathbb{D} \backslash\{0\}) \cap\left(W^{2,2} \cap W^{1, \infty}\right)(\mathbb{D})$ be a Willmore conformal branched immersion whose Gauss map $\vec{n}$ lies in $W^{1,2}(\mathbb{D})$ and with a branch point at 0 of multiplicity $\theta+1$. Let $\lambda$ be its conformal factor, $\vec{\gamma}_{0}$ the first residue defined as

$$
\overrightarrow{\gamma_{0}}:=\frac{1}{4 \pi} \int_{\partial \mathbb{D}} \vec{\nu} \cdot\left(\nabla \vec{H}-3 \pi_{\vec{n}}(\nabla \vec{H})+\nabla^{\perp} \vec{n} \times \vec{H}\right)
$$

Then there exists $\alpha \in \mathbb{Z}$ such that $\alpha \leq \theta$ and locally around the origin, $\vec{\Phi}$ has the following asymptotic expansion:

$$
\vec{\Phi}(z)=\Re\left(\vec{A} z^{\theta+1}+\sum_{j=1}^{\theta+1-\alpha} \vec{B}_{j} z^{\theta+1+j}+\vec{C}_{\alpha}|z|^{2(\theta+1)} z^{-\alpha}\right)-C \vec{\gamma}_{0}\left(\ln |z|^{2(m+1)}-4\right)+\vec{\xi}(z)
$$

where $\vec{B}_{j}, \vec{C}_{\alpha} \in \mathbb{C}^{3}$ are constant vectors, $\vec{A} \in \mathbb{C} \backslash\{0\}$, and $C \in \mathbb{R}$. Furthermore $\vec{\xi}$ satisfies the estimates

$$
\begin{aligned}
& \nabla^{j} \vec{\xi}(z)=O\left(|z|^{2(\theta+1)-\alpha-j+1-\kappa}\right) \text { for all } \kappa>0 \text { and } j \leq \theta+2-\alpha \\
& |z|^{-\theta} \nabla^{\theta-\alpha+3} \vec{\xi} \in L^{p} \text { for all } p<\infty
\end{aligned}
$$

In particular:

$$
\vec{H}(z)=\Re\left(\vec{E}_{\alpha} \bar{z}^{-\alpha}\right)-\vec{\gamma}_{0} \ln |z|+\vec{\eta}(z)
$$

where $\vec{E}_{\alpha} \in \mathbb{C}^{3} \backslash\{0\}$. The function $\vec{\eta}$ satisfies

$$
\begin{aligned}
& \nabla^{j} \vec{\eta}(z)=O\left(|z|^{1-j-\alpha-\kappa}\right) \text { for all } \kappa>0 \text { and } j \leq \theta-\alpha \\
& |z|^{\theta} \nabla^{\theta+1-\alpha} \vec{\eta} \in L^{p} \text { for all } p<\infty
\end{aligned}
$$


In the specific case of limits of Willmore immersions, the punctured disk described in theorem 1.4 is in fact the limit of simply connected disks, on which the first residue is null (see remark 1.1 in [12]). Since away from the concentration point $\nabla \vec{H}^{k}-3 \pi_{\vec{n}^{k}}\left(\nabla \vec{H}^{k}\right)+\nabla^{\perp} \vec{n}^{k} \times \vec{H}^{k}$ converges, the first residue around branch points of limit Willmore surfaces is always null. Such surfaces are called true Willmore surfaces. The quantity $\alpha$, although called the second residue (see definition 1.7 in [3] ), is not actually a residue and is thus not necessarily null. It will then take center stage in the study of limit Willmore surfaces. With this tool we can refine our understanding of the behavior around minimal concentration points with the following theorem:

Theorem 1.5. Let $\vec{\Phi}_{k}$ be a sequence of Willmore immersions of a closed surface $\Sigma$ satisfying the hypotheses of theorem 1.1. Then at each concentration point $p \in \Sigma$ of multiplicity $\theta_{p}+1$ on which a simple minimal bubble is blown, the second residue $\alpha_{p}$ of the limit immersion $\vec{\Phi}_{\infty}$ satisfies

$$
\alpha_{p} \leq \theta_{p}-1
$$

One should be aware that minimal bubbling is not necessarily simple. Indeed one could for instance imagine an Enneper surface bubbling on the branch point of a minimal surface of Enneper-Weierstrass data $(f, g)=\left(z^{2}, z\right)$, itself glued on a branch point of multiplicity 5 . However piling minimal spheres that way increases the total multiplicity. Minimal bubbling on branch points of multiplicity 3 is thus simple. Consequently theorem 1.5 allows us to eliminate some surfaces as a support for minimal bubbling.

Corollary 1.6. The convergence of Willmore immersions cannot lead to a minimal bubble and an inverted Chen-Gackstatter torus.

Let us recall that a Chen-Gackstatter torus is a minimal torus with one branched end, which is asymptotic to an Enneper end of multiplicity 3. Its inversion is thus a branched Willmore torus with one branch point of multiplicity 3 and Willmore energy $12 \pi$. We refer the reader to [8] or [17] for a complete study.

We can now extend theorem 1.2 .

Theorem 1.7. Let $\Sigma$ be a closed surface of genus 1 and $\vec{\Phi}_{k}: \Sigma \rightarrow \mathbb{R}^{3}$ a sequence of Willmore immersions such that the induced metric remains in a compact set of the moduli space and

$$
\limsup _{k \rightarrow \infty} W\left(\vec{\Phi}_{k}\right) \leq 12 \pi
$$

Then there exists a diffeomorphism $\psi_{k}$ of $\Sigma$ and a conformal transformation $\Theta_{k}$ of $\mathbb{R}^{3} \cup\{\infty\}$, such that $\Theta_{k} \circ \vec{\Phi}_{k} \circ \psi_{k}$ converges up to a subsequence toward a smooth Willmore immersion $\vec{\Phi}_{\infty}: \Sigma \rightarrow \mathbb{R}^{3}$ in $C^{\infty}(\Sigma)$.

Proof. We only have to exclude the case

$$
\limsup _{k \rightarrow \infty} W\left(\vec{\Phi}_{k}\right)=12 \pi
$$

Consider then $\vec{\Phi}_{k}$ satisfying (4) and converging toward $\vec{\Phi}_{\infty}$ away from a finite number of concentration points. We consider a concentration point and reason on its multiplicity $\theta_{0}+1$. If $\theta_{0} \geq 1$, using corollary 3.3 (see below), the bubble glued on its concentration point is branched, with the same multiplicity. Using proposition C.1 in 12 ensures that the multiplicity is odd, and then $\theta_{0} \geq 2$. Given P. Li and S. Yau's inequality (see 14]) and (4), $\vec{\Phi}_{\infty}$ has a Willmore energy of exactly $12 \pi$ meaning that the branch point is of multiplicity exactly 3 , that the bubbles have no Willmore energy (i.e. they are minimal and more accurately Enneper). Using formulas from [10, detailed in appendix (see propositions A.9 and A.10, $\vec{\Phi}_{\infty}$ is the inverse of a minimal torus of total curvature $-8 \pi$. The main result of [16] ensures that this minimal torus is a Chen-Gackstatter immersion. We are then in the case excluded by corollary 1.6

If the concentration point is not branched, we refer the reader to the concluding remark of $\mathrm{P}$. Laurain and T. Rivière's 12 (found just before the appendix) which states that the energy is then at least $\beta_{1}+12 \pi$, where $\beta_{1}$ is the infimum of the Willmore energy of Willmore tori. We would then be above our $12 \pi$ ceiling, which concludes the proof. 
The compactness of Willmore tori could fail with an energy strictly above $12 \pi$. One could imagine a sequence of non conformally minimal tori (similar to the ones described by U. Pinkall in [23]) of Willmore energy $12 \pi+\delta$ which degenerates into a branched torus of same energy, with an Enneper bubble. To avoid contradicting theorem 1.5, the branch point would need a second residue $\alpha \leq 1$. The existence of such a true Willmore torus is key in understanding compactness above $12 \pi$. In addition, one must notice that under the conclusion of theorem 1.5 . A. Michelat and T. Rivière, in [20, have proven that the Bryant's quartic, denoted $\mathcal{Q}$, of the limit surface is then holomorphic, meaning constant in the torus case. Since, according to R. Bryant's [7, $\mathcal{Q}=0$ implies that the surface is the inversion of a minimal surface, one could hope to push A. Michelat and T. Rivière's reasoning to the next order in the special case of a minimal bubble and conclude that the limit surface is an inversion of a minimal surface. Its energy would then be at least $16 \pi$, which would get us closer to the compactness strictly below $16 \pi$ to surfaces of genus lower than 1 . The possible counter example would be a $12 \pi$ branched minimal torus on which a non conformally minimal, non compact Willmore sphere is blown.

To extend the compactness below $12 \pi$ to immersions of a higher genus, it would be enough to show that the only minimal immersions of critical curvature are asymptotic to the Enneper surface near their branched end. This would be in agreement with the conjecture that the Chen-Gackstatter immersions are the only one with critical curvature for a given genus.

Section 2 will prove theorem 1.3 and build an exemple of minimal bubbling. Then section 3 will be devoted to translating theorem 1.5 in local conformal charts and to the possible adjustments, in notation or with the conformal group, that can be done to simplify the problem. Section 4 will give the first expansions on the conformal factor. Section 5 will prove theorem 1.5 and its corollaries.

Acknowledgments: The author would like to thank his advisor Paul Laurain for his support and precious advices. This work was partially supported by the ANR BLADE-JC ANR- 18-CE40-0023.

\section{Proof of theorem 1.3}

We will build a sequence of Willmore immersions whose energy $E$ concentrates on a point where an Enneper bubble blows up. Working from section 5 of R. Bryant's [7, we study a family of four ended minimal immersions $\vec{\Psi}_{\mu}: \mathbb{C} \backslash\left\{a_{1}, a_{2}, a_{2}\right\} \rightarrow \mathbb{R}^{3}:$

$$
\begin{aligned}
\vec{\Psi}_{\mu} & =2 \Re\left(f_{\mu}\right) \\
\vec{f}_{\mu} & =\frac{\vec{a}_{1}}{z-\mu}+\frac{\vec{a}_{2}}{z-\mu j}+\frac{\vec{a}_{3}}{z-\mu j^{2}}+\vec{a}_{4} z,
\end{aligned}
$$

with $\vec{a}_{1}, \vec{a}_{2}, \vec{a}_{3}, \vec{a}_{4} \in \mathbb{C}^{3}, j^{3}=1$, and $\mu$ a real parameter that will go toward 0 . As explained in [7] the $\left(\vec{a}_{i}\right)$ must be constrained for $\Psi_{\mu}$ to be a conformal immersion. Indeed:

$$
\begin{aligned}
\left\langle\left(\Psi_{\mu}\right)_{z},\left(\Psi_{\mu}\right)_{z}\right\rangle & =\left\langle\left(f_{\mu}\right)_{z},\left(f_{\mu}\right)_{z}\right\rangle \\
& =\frac{\left\langle\vec{a}_{1}, \vec{a}_{1}\right\rangle}{(z-\mu)^{4}}+\frac{\left\langle\vec{a}_{2}, \vec{a}_{2}\right\rangle}{(z-\mu j)^{4}}+\frac{\left\langle\vec{a}_{3}, \vec{a}_{3}\right\rangle}{\left(z-\mu j^{2}\right)^{4}}+\left\langle\vec{a}_{4}, \vec{a}_{4}\right\rangle \\
& +\frac{2\left\langle\vec{a}_{1}, \vec{a}_{2}\right\rangle}{(z-\mu)^{2}(z-\mu j)^{2}}+\frac{2\left\langle\vec{a}_{1}, \vec{a}_{3}\right\rangle}{(z-\mu)^{2}\left(z-\mu j^{2}\right)^{2}}+\frac{2\left\langle\vec{a}_{2}, \vec{a}_{3}\right\rangle}{(z-\mu j)^{2}\left(z-\mu j^{2}\right)^{2}} \\
& -\frac{2\left\langle\vec{a}_{1}, \vec{a}_{4}\right\rangle}{(z-\mu)^{2}}-\frac{2\left\langle\vec{a}_{2}, \vec{a}_{4}\right\rangle}{(z-\mu j)^{2}}-\frac{2\left\langle\vec{a}_{3}, \vec{a}_{4}\right\rangle}{\left(z-\mu j^{2}\right)^{2}} .
\end{aligned}
$$

Further since given $u, v \in \mathbb{C}$ :

$$
\frac{1}{(z-u)^{2}(z-v)^{2}}=\frac{1}{(u-v)^{2}} \frac{1}{(z-u)^{2}}+\frac{1}{(u-v)^{2}} \frac{1}{(z-v)^{2}}-\frac{2}{(u-v)^{3}} \frac{1}{z-u}+\frac{2}{(u-v)^{3}} \frac{1}{z-v},
$$

we deduce that $\left\langle\left(\Psi_{\mu}\right)_{z},\left(\Psi_{\mu}\right)_{z}\right\rangle=0$ if and only if

$$
\begin{aligned}
& \left\langle\vec{a}_{1}, \vec{a}_{1}\right\rangle=\left\langle\vec{a}_{2}, \vec{a}_{2}\right\rangle=\left\langle\vec{a}_{3}, \vec{a}_{3}\right\rangle=\left\langle\vec{a}_{4}, \vec{a}_{4}\right\rangle=0, \\
& \left\langle\vec{a}_{1}, \vec{a}_{2}\right\rangle=\left\langle\vec{a}_{1}, \vec{a}_{3}\right\rangle=\left\langle\vec{a}_{2}, \vec{a}_{3}\right\rangle \\
& \vec{a}_{4}=-\frac{1}{3 \mu^{2}}\left(\vec{a}_{1}+j \vec{a}_{2}+j^{2} \vec{a}_{3}\right) .
\end{aligned}
$$

One can check that under the conditions $[6),\left(\vec{a}_{1}, \vec{a}_{2}, \vec{a}_{3}\right)$ is a linearly independant family of $\mathbb{C}^{3}$ and thus that $\Psi_{\mu}$ is an immersion. 
Here we take, with $b \in \mathbb{C}$ a parameter to be adjusted later,

$$
\vec{a}_{1}=\frac{1}{2 \mu^{2}} \vec{e}_{0}, \vec{a}_{2}=\frac{j}{2 \mu^{2}} \vec{e}_{0}-\frac{\mu^{2} b^{2}}{2} \overline{\vec{e}_{0}}+b j^{2} \vec{e}_{3}, \vec{a}_{3}=\frac{j^{2}}{2 \mu^{2}} \vec{e}_{0}-\frac{\mu^{2} b^{2}}{2} \overline{\vec{e}_{0}}-b j \vec{e}_{3},
$$

where

$$
\vec{e}_{0}=\left(\begin{array}{l}
1 \\
i \\
0
\end{array}\right), \vec{e}_{3}=\left(\begin{array}{l}
0 \\
0 \\
1
\end{array}\right)
$$

One can check that these $\left(a_{i}\right)$ satisfy $(6)$. Computing, we find:

$$
\vec{f}_{\mu}=\frac{3}{z^{3}-\mu^{3}} \frac{1}{2} \vec{e}_{0}-b^{2}\left(\mu^{2} \frac{2 z+\mu}{z^{2}+\mu z+\mu^{2}}+\frac{z}{3}\right) \frac{1}{2} \overline{\vec{e}_{0}}+b j(j-1) \frac{z+\mu}{z^{2}+\mu z+\mu^{2}} \vec{e}_{3} .
$$

To simplify this expression we set $b=\frac{3 a}{2 j(j-1)}$ with $a \in \mathbb{C}$ to be fixed at the end of the reasoning, and reach:

$$
\vec{f}_{\mu}=\frac{3}{z^{3}-\mu^{3}} \frac{1}{2} \vec{e}_{0}+\frac{a^{2}}{4}\left(3 \mu^{2} \frac{2 z+\mu}{z^{2}+\mu z+\mu^{2}}+z\right) \frac{1}{2} \overline{\vec{e}_{0}}+\frac{3 a}{2} \frac{z+\mu}{z^{2}+\mu z+\mu^{2}} \vec{e}_{3} .
$$

Then $\vec{\Psi}_{\mu}: \mathbb{S}^{2} \rightarrow \mathbb{R}^{3}$ is a sequence of minimal immersions with four simple planar ends. Applying propositions A.9 and A.10 (see in the appendix) we find:

$$
\begin{gathered}
\int_{\mathbb{S}^{2}} K_{\vec{\Psi}_{\mu}} d \operatorname{vol}_{\vec{\Psi}_{\mu}}=-12 \pi, \\
\int_{\mathbb{S}^{2}}|\AA|_{\vec{\Psi}_{\mu}}^{2} d \operatorname{vol}_{g_{\mathbb{\Psi}_{\mu}}}=24 \pi .
\end{gathered}
$$

Letting $\mu \rightarrow 0$ in (7) we find that, away from 0 ,

$$
\vec{f}_{\mu} \rightarrow \vec{f}_{0}=\frac{3}{2 z^{3}} \vec{e}_{0}+\frac{a^{2} z}{8} \overrightarrow{e_{0}}+\frac{3 a}{2 z} \overrightarrow{e_{3}}
$$

and deduce that $\vec{\Psi}_{\mu} \rightarrow \vec{\Psi}_{0}:=2 \Re\left(\vec{f}_{0}\right)$ smoothly away from 0 , where $\vec{\Psi}_{0}$ is a branched minimal immersion of the sphere with one simple planar end and one planar end of multiplicity 3. This immersion is in fact the López minimal surface mentioned in theorem 1.3 . Then

$$
\begin{gathered}
\int_{\mathbb{S}^{2}} K_{\vec{\Psi}_{0}} d \operatorname{vol}_{\vec{\Psi}_{0}}=-8 \pi, \\
\int_{\mathbb{S}^{2}}|\stackrel{\AA}{A}|_{\vec{\Psi}_{0}}^{2} d \operatorname{vol}_{g_{\vec{\Psi}_{0}}}=16 \pi .
\end{gathered}
$$

Let $p$ be a point in $\mathbb{R}^{3}$ such that $d\left(p, \vec{\Psi}_{\mu}\right)>1$. We now introduce $\vec{\Phi}_{\mu}:=\iota_{p} \circ \vec{\Psi}_{\mu}$ and $\vec{\Phi}_{0}:=\iota_{p} \circ \vec{\Psi}_{0}$, with $\iota(x)=\frac{x-p}{|x-p|^{2}}$ the inversion in $\mathbb{R}^{3}$ centered at $p$. Then $\vec{\Phi}_{\mu}$ is a sequence of closed Willmore conformal immersions of the sphere converging toward $\vec{\Phi}_{0}$ smoothly away from 0 , and $\vec{\Phi}_{0}$ is a closed Willmore conformal branched immersion of the sphere with a single branch point of multiplicity 3 at 0 . Thus

$$
\begin{gathered}
\int_{\mathbb{S}^{2}} K_{\vec{\Phi}_{\mu}} d \operatorname{vol}_{\vec{\Phi}_{\mu}}=4 \pi, \\
\int_{\mathbb{S}^{2}} K_{\vec{\Phi}_{0}} d \operatorname{vol}_{g_{\vec{\Phi}_{0}}}=8 \pi .
\end{gathered}
$$

Since $|\stackrel{\AA}{A}|^{2} d \operatorname{vol}_{g}$ is a conformal invariant, we deduce from $(9)$ and 12$]$ :

$$
\begin{gathered}
\int_{\mathbb{S}^{2}}|\AA|_{\vec{\Phi}_{\mu}}^{2} d \operatorname{vol}_{\vec{\Phi}_{\mu}}=24 \pi, \\
\int_{\mathbb{S}^{2}}|\AA|_{\vec{\Phi}_{0}}^{2} d \operatorname{vol}_{\vec{\Phi}_{\vec{\Phi}_{0}}}=16 \pi .
\end{gathered}
$$


With proposition A.10 (see in the appendix) we conclude with 13 and 15 :

$$
\int_{\mathbb{S}^{2}} H_{\vec{\Phi}_{\mu}}^{2} d \operatorname{vol}_{\vec{\Phi} \mu}=\frac{1}{2} \int_{\mathbb{S}^{2}}|\AA|_{\vec{\Phi}_{\mu}}^{2} d \operatorname{vol}_{g_{\vec{\Phi}_{\mu}}}+\int_{\mathbb{S}^{2}} K_{\vec{\Phi}_{\mu}} d \operatorname{vol}_{\vec{\Phi}_{\mu}}=16 \pi,
$$

and with (14) and (16):

$$
\int_{\mathbb{S}^{2}} H_{\vec{\Phi}_{0}}^{2} d \operatorname{vol}_{\vec{\Phi}_{0}}=\frac{1}{2} \int_{\mathbb{S}^{2}}|\AA|_{\vec{\Phi}_{0}}^{2} d \operatorname{vol}_{\vec{\Phi}_{0}}+\int_{\mathbb{S}^{2}} K_{\vec{\Phi}_{0}} d \operatorname{vol}_{\vec{\Phi}_{0}}=16 \pi
$$

Comparing (15)-(18) reveals that while: $W\left(\vec{\Phi}_{\mu}\right) \rightarrow W\left(\vec{\Phi}_{0}\right)$, there is an energy gap of $8 \pi$ in $\mathcal{E}$ (or equivalently in $E$ ). From this, and the energy quantization theorem (theorem 1.1. written above), we deduce that a simple minimal bubble of energy $E=8 \pi$ is blown. The only possible bubble is then an Enneper surface (see for instance [22]), given by:

$$
E(z)=2 \Re\left(\frac{z}{2} \vec{e}_{0}+\frac{z^{2}}{2} \vec{e}_{3}-\frac{z^{3}}{6} \overrightarrow{\vec{e}_{0}}\right) .
$$

This is enough to ensure that the immersions $\vec{\Phi}_{\mu}$ offer an exemple of an Enneper bubble appearing on a sequence of Willmore immersions, which proves theorem 1.3

We however wish to make the appearance of the Enneper bubble explicit in the computations. To do that we will perform a blow-up at the origin at scale $\mu^{3}$. This concentration scale has been determined the classical way (see the bubble tree extraction procedure in [12] or [4]) by computing $\left\|\nabla \vec{n}_{\vec{\Phi}_{\mu}}\right\|_{L^{\infty}\left(\mathbb{S}^{2}\right)}$. Since these computations do not by themselves further the understanding of the bubbling phenomena, they are omitted. Considering (7) we find

$$
\begin{aligned}
\vec{f}_{\mu}\left(\mu^{3} z\right) & =\frac{3}{2 \mu^{3}\left(\mu^{6} z^{3}-1\right)} \vec{e}_{0}+\frac{a^{2} \mu}{8}\left(3 \frac{2 z \mu^{2}+1}{1+\mu^{2} z+\mu^{4} z^{2}}+\mu^{2} z\right) \overrightarrow{\vec{e}_{0}} \\
& +\frac{3 a}{2 \mu} \frac{1+\mu^{2} z}{1+\mu^{2} z+\mu^{4} z^{2}} \vec{e}_{3} \\
& =\left(-\frac{3}{2 \mu^{3}}-\frac{3 \mu^{3} z^{3}}{2}+O\left(\mu^{9}\right)\right) \vec{e}_{0}+\frac{a^{2}}{2}\left(\frac{3 \mu}{4}+z \mu^{3}-+O\left(\mu^{5}\right)\right) \overrightarrow{\vec{e}_{0}} \\
& +a\left(\frac{3}{2 \mu}-\frac{3}{2} \mu^{3} z^{2}+O\left(\mu^{5}\right)\right) \vec{e}_{3} .
\end{aligned}
$$

Which means that

$$
\begin{aligned}
\vec{\Psi}_{\mu}\left(\mu^{3} z\right) & =\frac{1}{2}\left(\frac{3 \bar{a}^{2} \mu}{4}-\frac{3}{\mu^{3}}+\mu^{3}\left(\bar{a}^{2} \bar{z}-3 z^{3}\right)+O\left(\mu^{5}\right)\right) \vec{e}_{0} \\
& +\frac{1}{2}\left(\frac{3 a^{2} \mu}{4}-\frac{3}{\mu^{3}}+\mu^{3}\left(a^{2} z-3 \bar{z}^{3}\right)+O\left(\mu^{5}\right)\right) \overline{\vec{e}_{0}} \\
& +\left(\frac{3(a+\bar{a})}{2 \mu}-\frac{3 \mu^{3}}{2}\left(a z^{2}+\bar{a} \bar{z}^{2}\right)+O\left(\mu^{5}\right)\right) \vec{e}_{3} .
\end{aligned}
$$

With $p=\frac{p_{1}}{2} \vec{e}_{0}+\frac{\overline{p_{1}}}{2} \overrightarrow{e_{0}}+p_{3} \overrightarrow{e_{3}}$ defined previously we conclude:

$$
\begin{aligned}
\vec{\Psi}_{\mu}\left(\mu^{3} z\right)-p & =\frac{1}{\mu^{3}}\left(\frac{1}{2}\left(-3-\mu^{3} p_{1}+\frac{3 \bar{a}^{2} \mu^{4}}{4}+\mu^{6}\left(\bar{a}^{2} \bar{z}-3 z^{3}\right)+O\left(\mu^{8}\right)\right)\left(\begin{array}{c}
1 \\
i \\
0
\end{array}\right)\right. \\
& +\frac{1}{2}\left(-3-\mu^{3} \overline{p_{1}}+\frac{3 a^{2} \mu^{4}}{4}+\mu^{6}\left(a^{2} z-3 \bar{z}^{3}\right)+O\left(\mu^{8}\right)\right)\left(\begin{array}{c}
1 \\
-i \\
0
\end{array}\right) \\
& \left.+\left(\frac{3(a+\bar{a}) \mu^{2}}{2}-\mu^{3} p_{3}-\frac{3 \mu^{6}}{2}\left(a z^{2}+\bar{a} \bar{z}^{2}\right)+O\left(\mu^{8}\right)\right) \vec{e}_{3}\right) .
\end{aligned}
$$

Here the only relevant terms are the first non constant ones i.e. those in $\mu^{6}$. This yields:

$$
\begin{aligned}
\left|\vec{\Psi}_{\mu}\left(\mu^{3} z\right)-p\right|^{2} & =\frac{1}{\mu^{6}}\left(9+3 \mu^{3}\left(p_{1}+\overline{p_{1}}\right)+\mu^{4} \frac{9\left(a^{2}+\bar{a}^{2}\right)}{4}+\mu^{4} \frac{9(a+\bar{a})^{2}}{4}\right. \\
& -\frac{3 \mu^{5}(a+\bar{a})}{2}\left(p_{3}+\overline{p_{3}}\right)-3 \mu^{6}\left(a^{2} z+\bar{a}^{2} \bar{z}^{-} 3 z^{3}-3 \bar{z}^{3}+\left|p_{1}\right|^{2}+\left|p_{3}\right|^{2}\right) \\
& \left.+O\left(\mu^{7}\right)\right)
\end{aligned}
$$


We can combine 20) and (21):

$$
\begin{aligned}
\vec{\Phi}_{\mu}\left(\mu^{3} z\right)= & \frac{\vec{\Psi}_{\mu}-p}{\left|\vec{\Psi}_{\mu}-p\right|^{2}} \\
& =\mu^{3}\left(\frac{1}{2}\left(-\frac{1}{3}-\frac{\mu^{3}}{9} p_{1}+\frac{\bar{a}^{2} \mu^{4}}{12}+\frac{\mu^{6}}{9}\left(\bar{a}^{2} \bar{z}-3 z^{3}\right)+O\left(\mu^{7}\right)\right) \vec{e}_{0}\right. \\
& +\frac{1}{2}\left(-\frac{1}{3}-\frac{\mu^{3}}{9} \overline{p_{1}}+\frac{a^{2} \mu^{4}}{12}+\frac{\mu^{6}}{9}\left(a^{2} z-3 \bar{z}^{3}\right)+O\left(\mu^{7}\right)\right) \overline{\vec{e}_{0}} \\
& \left.+\left(\frac{a+\bar{a} \mu^{2}}{6}-\frac{\mu^{3}}{9} p_{3}-\frac{\mu^{6}}{6}\left(a z^{2}+\bar{a} \bar{z}^{2}\right)+O\left(\mu^{7}\right)\right) \vec{e}_{3}\right)\left(1-\frac{1}{3} \mu^{3}\left(p_{1}+\overline{p_{1}}\right)\right. \\
& -\mu^{4} \frac{\left(a^{2}+\bar{a}^{2}\right)}{4}-\mu^{4} \frac{(a+\bar{a})^{2}}{4}+\frac{\mu^{5}(a+\bar{a})}{6}\left(p_{3}+\overline{p_{3}}\right) \\
& \left.+\frac{1}{3} \mu^{6}\left(a^{2} z+\bar{a}^{2} \bar{z}-3 z^{3}-3 \bar{z}^{3}+\left|p_{1}\right|^{2}+\left|p_{3}\right|^{2}+\frac{1}{3}\left(p_{1}+\overline{p_{1}}\right)^{2}\right)+O\left(\mu^{7}\right)\right) \\
& =\vec{\Phi}_{\mu}(0)+\mu^{9}\left(\frac{1}{9}\left(\bar{a}^{2} \bar{z}-3 z^{3}-a^{2} z-\bar{a}^{2} \bar{z}+3 z^{3}+3 \bar{z}^{3}\right) \frac{1}{2} \vec{e}_{0}\right. \\
& \left.+\frac{1}{9}\left(a^{2} z-3 \bar{z}^{3}-a^{2} z-\bar{a}^{2} \bar{z}+3 z^{3}+3 \bar{z}^{3}\right) \frac{1}{2} \overline{\vec{e}_{0}}-\frac{a z^{2}+\bar{a} \bar{z}^{2}}{6} \vec{e}_{3}\right) \\
& +O\left(\mu^{10}\right) \\
& =\vec{\Phi}_{\mu}(0)+\mu^{9}\left(\left(\frac{\bar{z}^{3}}{3}-\frac{a^{2}}{9} z\right) \frac{1}{2} \vec{e}_{0}+\left(\frac{z^{3}}{3}-\frac{a^{2}}{9} \bar{z}\right) \frac{1}{2} \overline{\vec{e}_{0}}\right. \\
& \left.-\left(\frac{z^{2}}{3}+\frac{\bar{a}}{3} \frac{\bar{z}^{2}}{2}\right) \vec{e}_{3}\right)+O\left(\mu^{10}\right) .
\end{aligned}
$$

Taking $a=3$ we find exactly

$$
\vec{\Phi}_{\mu}\left(\mu^{3} z\right)=\vec{\Phi}_{\mu}(0)-\mu^{9} E(z)+O\left(\mu^{10}\right)
$$

Hence we do have:

$$
\frac{\vec{\Phi}_{\mu}\left(\mu^{3} z\right)-\vec{\Phi}_{\mu}(0)}{-\mu^{9}} \rightarrow E(z)
$$

smoothly on every compact of $\mathbb{C}$, which does illustrate theorem 1.3 .

Remark 2.0.1. Chosing another value for $a$ would have led to another Enneper surface, with EnneperWeierstrass data $(f, g)=\left(1, \frac{3}{a} z\right)$ instead of simply $(f, g)=(1, z)$.

Remark 2.0.2. One must notice the fundamentally asymetric role of $\vec{\Phi}_{0}$ (the surface) and $E$ (the bubble). Indeed while we have compactly glued $E$ on $\vec{\Phi}_{0}$ we cannot compactly glue $\vec{\Psi}_{0}=\iota \circ \vec{\Phi}_{0}$ on an inverted Enneper using the same construction, since $\Psi_{0}$ has an end which is not on the concentration point. Doing so would require to glue a closed bubble tree on said planar end (and would necessarily add Willmore energy to the concentration point). Furthermore, theorem 1.5 ensures that no construction will ever enable us to do so, given that the second residue of the inverted Enneper surface is $\alpha=2$ (see [3]). 

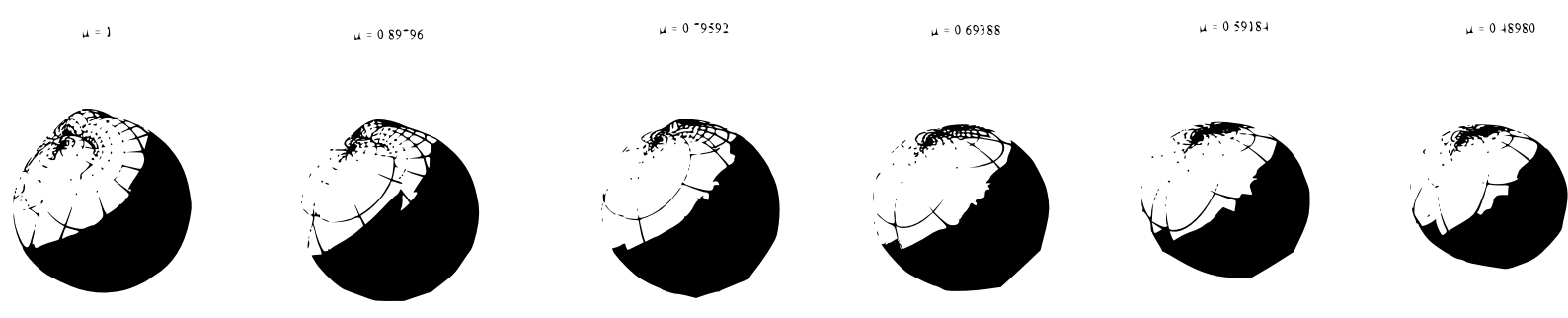

$\mu=038^{\cdots-6}$

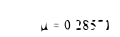

$\mu=01836^{-}$

$\mu=0083633$

$\mu=0040816$

$\mu=0020108$
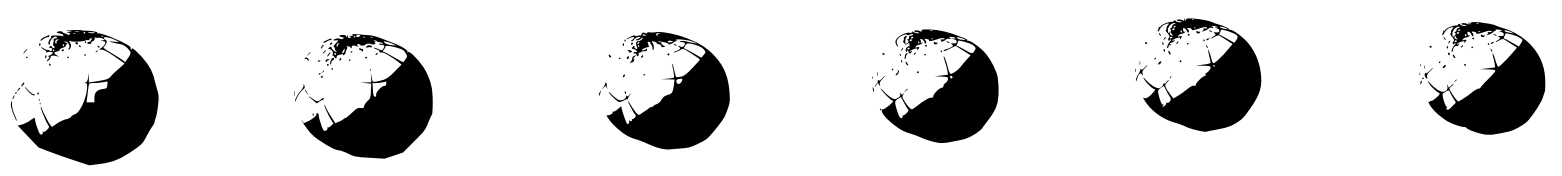

Figure 1. The transformation of the Bryant's surfaces into a López surface
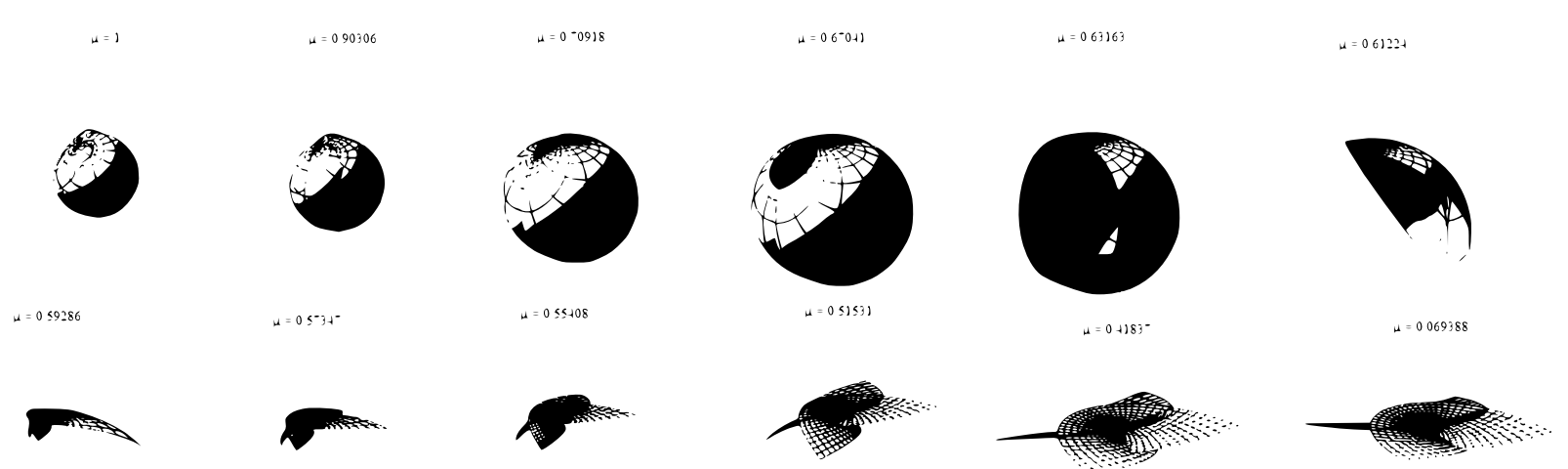

Figure 2. The blow-up into an Enneper surface

\section{Setting for a proof of theorem 1.5 in local conformal charts:}

While theorem 1.5 is stated globally, its conclusion is localized on the neighborhoods of concentration points on which a simple minimal bubble is blown. We will then work in conformal charts around such points. The aim of this section is to draw a set of hypotheses that these maps may satisfy, sometimes up to slight adjustments that can be done without loss of generality. We will also delve into the first consequences of these hypotheses to clarify our framework.

\subsection{Convergence in local conformal charts:}

We here wish to show the following:

Lemma 3.1. Let $\xi_{k}$ be a sequence of Willmore immersions of a closed surface $\Sigma$ satisfying the hypotheses of theorem 1.1. Then, in proper conformal charts around a concentration point on which a simple minimal bubble is blown, $\xi^{k}$ yields a sequence of Willmore conformal immersions $\vec{\Phi}^{\varepsilon}: \mathbb{D} \rightarrow \mathbb{R}^{3}$, of conformal factor $\lambda^{\varepsilon}=\frac{1}{2} \ln \left(\frac{\left|\nabla \vec{\Phi}^{\varepsilon}\right|^{2}}{2}\right)$, Gauss map $\vec{n}^{\varepsilon}$, mean curvature $H^{\varepsilon}$ and tracefree curvature $\Omega^{\varepsilon}:=2\left\langle\vec{\Phi}_{z z}^{\varepsilon}, \vec{n}^{\varepsilon}\right\rangle$, satisfying the following set of hypotheses:

1. There exists $C_{0}>0$ such that

$$
\left\|\vec{\Phi}^{\varepsilon}\right\|_{L^{\infty}(\mathbb{D})}+\left\|\nabla \vec{\Phi}^{\varepsilon}\right\|_{L^{2}(\mathbb{D})}+\left\|\nabla \lambda^{\varepsilon}\right\|_{L^{2, \infty}(\mathbb{D})}+\left\|\nabla \vec{n}^{\varepsilon}\right\|_{L^{2}(\mathbb{D})} \leq C_{0} .
$$

2. $\vec{\Phi}^{\varepsilon} \rightarrow \vec{\Phi}^{0} C_{\text {loc }}^{\infty}(\mathbb{D} \backslash\{0\})$, where $\vec{\Phi}^{0}$ is a true branched Willmore conformal immersion, with a unique branch point of multiplicity $\theta_{0}+1$ at 0 , meaning that

$$
\vec{\Phi}_{z}^{0} \sim_{0} \vec{A} z^{\theta_{0}} .
$$

We denote $\lambda^{0}$ its conformal factor, $\vec{n}^{0}$ its Gauss map, $H^{0}$ its mean curvature and $\Omega^{0}$ its tracefree curvature. 
3. There exists a sequence of real numbers $C^{\varepsilon}>0$ such that

$$
\widetilde{\Phi}^{\varepsilon}:=\frac{\vec{\Phi}^{\varepsilon}(\varepsilon \cdot)-\vec{\Phi}^{\varepsilon}(0)}{C^{\varepsilon}} \rightarrow \vec{\Phi}_{z}^{1}
$$

$C_{\text {loc }}^{\infty}(\mathbb{C})$, where $\vec{\Phi}^{1}$ is assumed to be a minimal conformal immersion of $\mathbb{C}$ with a branched end of multiplicity $\theta_{1}+1$, meaning that:

$$
\vec{\Phi}_{z}^{1} \sim \infty \widetilde{A} z^{\theta_{1}}
$$

We denote $\lambda^{1}$ its conformal factor, $\vec{n}^{1}$ its Gauss map, $H^{1}$ its mean curvature and $\Omega^{1}$ its tracefree curvature.

4.

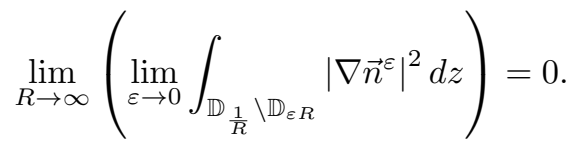

5. $\left|\Omega^{\varepsilon} e^{-\lambda^{\varepsilon}}\right|$ reaches its maximum at 0 and

$$
\left|\Omega^{\varepsilon} e^{-\lambda^{\varepsilon}}\right|(0)=\frac{2}{\varepsilon}
$$

Proof. Such assumptions are natural if we consider $\vec{\xi}^{k}$ satisfying the hypotheses of theorem 1.1 . Thanks to theorems I.2 and I.3 of 4 such a sequence $\vec{\xi}^{k}$ converges smoothly away from concentration points. In a conformal chart centered on a concentration point, $\vec{\xi}^{k}$ yields a sequence of conformal, weak Willmore immersions $\vec{\Phi}^{k}: \mathbb{D} \rightarrow \mathbb{R}^{3}$ converging smoothly away from the origin toward a true Willmore surface (i.e. hypothesis 2 ). Hypothesis 1 stands if we choose proper conformal charts (see theorem 3.1 of P. Laurain and T. Rivière's [13. for a detailed explanation).

Hypothesis 3 then specifies that we consider the case where there is only one simple minimal bubble which concentrates on 0 in the aforementioned chart. Hypothesis 4 is just the energy quantization once the whole bubble tree is extracted and corresponds to inequality VIII.8 in [4. Moreover, by definition of a concentration point

$$
\left\|\nabla \vec{n}^{k}\right\|_{L^{\infty}(\mathbb{D})} \rightarrow \infty
$$

On the other hand, the main result of [19] (namely inequality (96)) states that

$$
\left\|H^{k} \nabla \vec{\Phi}^{k}\right\|_{L^{\infty}(\mathbb{D})} \leq C\left(C_{0}\right) .
$$

Since $\left|\nabla \vec{n}^{k}\right|^{2}=\left|H^{k} \nabla \vec{\Phi}^{k}\right|^{2}+\left|\Omega^{k} e^{-\lambda^{k}}\right|^{2}$, necessarily

$$
\left\|\Omega^{k} e^{-\lambda^{k}}\right\|_{L^{\infty}(\mathbb{D})} \rightarrow \infty
$$

We then define the concentration speed as

$$
\varepsilon_{k}=\frac{2}{\left\|\Omega^{k} e^{-\lambda^{k}}\right\|_{L^{\infty}(\mathbb{D})}},
$$

and we assume it is reached at the origin. For simplicity's sake we reparametrize this sequence by the concentration speed which we denote $\varepsilon$. Hypothesis 5 is then a consequence of this slight adjustment.

An immediate consequence of hypotheses 2,4 is the following energy quantization result

$$
\int_{\mathbb{D}}\left|\nabla \vec{n}^{\varepsilon}\right|^{2} d z \rightarrow \int_{\mathbb{D}}\left|\nabla \vec{n}^{0}\right|^{2} d z+\int_{\mathbb{C}}\left|\nabla \vec{n}^{1}\right|^{2} d z .
$$

while hypothesis 1 ensures

$$
\left\|\nabla \vec{n}^{0}\right\|_{L^{2}(\mathbb{D})}+\left\|\nabla \vec{n}^{1}\right\|_{L^{2}(\mathbb{C})} \leq C\left(C_{0}\right)
$$


Further, if we denote $\widetilde{\lambda}^{\varepsilon}$ the conformal factor of $\widetilde{\Phi}^{\varepsilon}, \widetilde{H}^{\varepsilon}$ its mean curvature, $\widetilde{\Omega}^{\varepsilon}$ its tracefree curvature and $\widetilde{\vec{n}}^{\varepsilon}$ its Gauss map, we have

$$
\widetilde{\lambda}^{\varepsilon}=\lambda^{\varepsilon}(\varepsilon \cdot)-\ln \left(\frac{C^{\varepsilon}}{\varepsilon}\right)
$$

and

$$
\widetilde{\Omega}^{\varepsilon} e^{-\widetilde{\lambda}^{\varepsilon}}=\varepsilon\left[\Omega^{\varepsilon} e^{-\lambda^{\varepsilon}}\right](\varepsilon \cdot) .
$$

Using hypothesis 5 one may conclude that

$$
\left|\widetilde{\Omega}^{\varepsilon} e^{-\widetilde{\lambda}^{\varepsilon}}\right|(0)=2
$$

Hypothesis 3 then yields

$$
\left|\Omega^{1} e^{-\lambda^{1}}\right|(0)=2 .
$$

Similarly we know, thanks to hypotheses 3 and 5 .

$$
\left(\left|\widetilde{\Omega}^{\varepsilon} e^{-\widetilde{\lambda}^{\varepsilon}}\right|\right)_{z}(0)=0
$$

and:

$$
\left(\left|\Omega^{1} e^{-\lambda^{1}}\right|\right)_{z}(0)=0
$$

Finally hypothesis 4 allows us to apply theorem 1.4 to $\vec{\Phi}^{0}$ : there exists $\alpha \leq \theta_{0}, \vec{A} \in \mathbb{C}^{3} \backslash\{0\}$, $\left(\vec{B}_{j}\right)_{j=1 . . \theta_{0}+1-\alpha} \in \mathbb{C}^{3}, \vec{C}_{\alpha} \in \mathbb{C}^{3} \backslash\{0\}$ and $\xi: \mathbb{D} \rightarrow \mathbb{R}^{3}$ such that

$$
\vec{\Phi}_{z}^{0}=\vec{A} z^{\theta_{0}}+\sum_{j=1}^{\theta_{0}+1-\alpha} \vec{B}_{j} z^{\theta_{0}+j}+\frac{\vec{C}_{\alpha}}{\theta_{0}+1} z^{\theta_{0}-\alpha} \bar{z}^{\theta_{0}+1}+\frac{\overrightarrow{\vec{C}_{\alpha}}}{\theta_{0}+1-\alpha} z^{\theta_{0}} \bar{z}^{\theta_{0}+1-\alpha}+\vec{\xi}_{z},
$$

where $\xi$ satisfies:

$$
\nabla^{j} \vec{\xi}=O\left(r^{2 \theta_{0}+3-\alpha-j-\kappa}\right)
$$

for all $\kappa>0$ and $j \leq \theta_{0}+2-\alpha$. The second residue $\alpha$ is in fact defined as follows (see theorem I.8 in [3]):

$$
H^{0} \sim C_{\alpha}|z|^{-\alpha} .
$$

Our proofs will use the quantities $\vec{L}, S$ and $\vec{R}$, stemming from the Willmore conservation laws (see for instance theorem I.4 in [24]), which at the core, are a consequence of the conformal invariance of $W$ (see [2]). More precisely $\vec{L}, S$ and $\vec{R}$ are defined as follows:

$$
\begin{aligned}
\nabla^{\perp} \vec{L} & =\nabla \vec{H}-3 \pi_{\vec{n}}(\nabla \vec{H})+\nabla^{\perp} \vec{n} \times \vec{H}, \\
\nabla^{\perp} S & =\left\langle\vec{L}, \nabla^{\perp} \vec{\Phi}\right\rangle, \\
\nabla^{\perp} \vec{R} & =\vec{L} \times \nabla^{\perp} \vec{\Phi}+2 H \nabla^{\perp} \vec{\Phi} .
\end{aligned}
$$

Exploiting these was key in T. Rivière's proof of the $\varepsilon$-regularity for Willmore surfaces.

Under hypotheses 1 155, the conclusion of [19] stands and yields (see (96)-(98) in the aforementioned paper):

$$
\begin{gathered}
\left\|H^{\varepsilon} \nabla \vec{\Phi}^{\varepsilon}\right\|_{L^{\infty}(\mathbb{D})} \leq C\left(C_{0}\right), \\
\left\|\nabla \vec{\Phi}^{\varepsilon}\right\|_{W^{3, p}(\mathbb{D})} \leq C\left(C_{0}\right),
\end{gathered}
$$

while the second and third Willmore quantities satisfy

$$
\left\|\nabla S^{\varepsilon}\right\|_{W^{1, p}(\mathbb{D})}+\left\|\nabla \vec{R}^{\varepsilon}\right\|_{W^{1, p}(\mathbb{D})} \leq C\left(C_{0}\right)
$$

for all $p<\infty$. Up to an inconsequential translation one can further assume $\vec{\Phi}^{\varepsilon}(0)=0$. 


\subsection{Branch point-branched end correspondence:}

The goal of this subsection is to show the equality of the multiplicity of the end of the bubble and the multiplicity of the branch point of the surface:

Theorem 3.2. Let $\vec{\Phi}^{\varepsilon}: \mathbb{D} \rightarrow \mathbb{R}^{3}$ satisfying $1-5$. Then $\theta_{0}=\theta_{1}=\theta$.

Proof. Since $\vec{\Phi}^{\varepsilon}$ is conformal, the Liouville equation states

$$
\Delta \lambda^{\varepsilon}=K^{\varepsilon} e^{2 \lambda^{\varepsilon}}
$$

where $K^{\varepsilon}$ is the Gauss curvature of $\vec{\Phi}^{\varepsilon}$. Then given $R \in \mathbb{R}_{+}$

$$
\begin{aligned}
& \int_{\mathbb{D}_{\frac{1}{R}} \backslash \mathbb{D}_{\varepsilon R}} K^{\varepsilon} e^{2 \lambda^{\varepsilon}} d z=\int_{\mathbb{D}_{\frac{1}{R} \backslash \mathbb{D}_{\varepsilon R}}} \Delta \lambda^{\varepsilon} d z=\int_{\partial \mathbb{D}_{\frac{1}{R}}} \partial_{r} \lambda^{\varepsilon} d \sigma-\int_{\partial \mathbb{D}_{\varepsilon R}} \partial_{r} \lambda^{\varepsilon} d \sigma \\
& =\int_{\partial \mathbb{D}_{\frac{1}{R}}} \partial_{r} \lambda^{\varepsilon} d \sigma-\int_{\partial \mathbb{D}_{R}} \varepsilon \partial_{r} \lambda^{\varepsilon}(\varepsilon \cdot) d \sigma \\
& =\int_{\partial \mathbb{D}_{\frac{1}{R}}} \partial_{r} \lambda^{\varepsilon} d \sigma-\int_{\partial \mathbb{D}_{R}} \partial_{r} \widetilde{\lambda}^{\varepsilon} d \sigma .
\end{aligned}
$$

Besides, hypotheses 2 and 3 ensure that $\lambda^{\varepsilon} \rightarrow \lambda^{0}$ on $\partial \mathbb{D}_{\frac{1}{R}}$ and $\widetilde{\lambda}^{\varepsilon} \rightarrow \lambda^{1}$ on $\partial \mathbb{D}_{R}$. In addition, since $\vec{\Phi}^{0}$ has a branch point of multiplicity $\theta_{0}+1$ at 0 ,

$$
\lim _{R \rightarrow \infty} \int_{\mathbb{D}_{\frac{1}{R}}} \partial_{r} \lambda^{0} d \sigma \rightarrow 2 \pi \theta_{0}
$$

Similarly $\vec{\Phi}^{1}$ has an end of multiplicity $\theta_{1}+1$ at $\infty$, which implies:

$$
\lim _{R \rightarrow \infty} \int_{\mathbb{D}_{R}} \partial_{r} \lambda^{1} d \sigma \rightarrow 2 \pi \theta_{1}
$$

Injecting (40) and 417) in 39 yields

$$
\begin{aligned}
2 \pi\left|\theta_{0}-\theta_{1}\right| & \leq \lim _{R \rightarrow \infty}\left(\lim _{\varepsilon \rightarrow 0}\left|\int_{\partial \mathbb{D}_{\frac{1}{R}}} \partial_{r} \lambda^{\varepsilon} d \sigma-\int_{\partial \mathbb{D}_{R}} \partial_{r} \widetilde{\lambda}^{\varepsilon} d \sigma\right|\right) \\
& \leq \lim _{R \rightarrow \infty}\left(\lim _{\varepsilon \rightarrow 0}\left|\int_{\mathbb{D}_{\frac{1}{R}} \backslash \mathbb{D}_{\varepsilon R}} K^{\varepsilon} e^{2 \lambda^{\varepsilon}} d z\right|\right) \leq \lim _{R \rightarrow \infty}\left(\lim _{\varepsilon \rightarrow 0} \int_{\mathbb{D}_{\frac{1}{R}} \backslash \mathbb{D}_{\varepsilon R}}\left|K^{\varepsilon} e^{2 \lambda^{\varepsilon}}\right| d z\right) \\
& \leq \lim _{R \rightarrow \infty}\left(\lim _{\varepsilon \rightarrow 0} \int_{\left.\mathbb{D}_{\frac{1}{R} \backslash \mathbb{D}_{\varepsilon R}}\left|\nabla \vec{n}^{\varepsilon}\right|^{2} d z\right)=0,}\right.
\end{aligned}
$$

using hypothesis 1 . As a conclusion $\theta_{0}=\theta_{1}=\theta$.

While we wrote the proof in the case specific to our paper, it remains valid for any behavior of $\vec{\Phi}^{0}$ and $\vec{\Phi}^{1}$ (branched points or ends) and relies solely on the energy quantization result. In a broader frame this corresponds to the following:

Corollary 3.3. A Willmore bubble with a branched end of multiplicity $\theta+1$ at infinity can only appear on a branch point of multiplicity $\theta+1$.

A Willmore bubble with a branch point of multiplicity $\theta-1$ at infinity can only appear on a branched end of multiplicity $\theta-1$.

Here, it must be emphasized that the true invariant is the behavior of the conformal factor, transmitted from the bubble to the surface. It then translates as information on the multiplicity of the respective singular points, depending on their nature (branch point or end) and their location (at $\infty$ or in a compact). 


\subsection{Macroscopic adjustments}

This section is dedicated to the proof of the following result:

Lemma 3.4. Let $\vec{\Phi}^{\varepsilon}: \mathbb{D} \rightarrow \mathbb{R}^{3}$ be a sequence of Willmore conformal immersions satisfying hypotheses 1.5 . Then $\theta$ is even, and up to macroscopic adjustments we can assume that:

6.

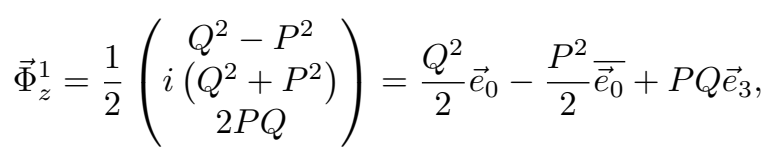

where $P, Q \in \mathbb{C}_{\frac{\theta}{2}}[X], P \wedge Q=1$, and

$$
P(0)=P^{\prime \prime}(0)-2 Q^{\prime}(0)=0, Q(0)=P^{\prime}(0)=1
$$

The end of multiplicity $\theta+1$ of $\vec{\Phi}^{1}$ can be highlighted as follows: there exists $\widetilde{A} \in \mathbb{C}^{3} \backslash\{0\}$ and $\vec{V} \in \mathbb{C}_{\theta-1}[X]$ such that

$$
\vec{\Phi}_{z}^{1}=\widetilde{A} z^{\theta}+\vec{V}
$$

Here we used $\mathbb{C}[X]$ to denote polynomials with complex coefficients, while for any $m \in \mathbb{N}, \mathbb{C}_{m}[X]$ denotes the space of polynomials of degree lower or equal to $m$.

\section{Proof. Adjusting with homothetic transformations of $\mathbb{R}^{3}$ :}

Since $\vec{\Phi}^{1}$ has no branch point on $\mathbb{C}$, up to a fixed rotation and a dilation in $\mathbb{R}^{3}$ one can assume:

$$
\vec{\Phi}_{z}^{1}(0)=\frac{1}{2}\left(\begin{array}{l}
1 \\
i \\
0
\end{array}\right)
$$

\section{Adjusting the parametrization:}

Taking $M_{-\omega}=\left(\begin{array}{ccc}\cos \omega & -\sin \omega & 0 \\ \sin \omega & \cos \omega & 0 \\ 0 & 0 & 1\end{array}\right)$ with $\omega \in \mathbb{R}$ to be determined at a later point, we set $\vec{\Psi}:=M_{-\omega} \vec{\Phi}^{1}\left(e^{i \omega} \cdot\right)$. We denote respectively $\lambda_{\vec{\Psi}}, H_{\vec{\Psi}}, \Omega_{\vec{\Psi}}$ and $\vec{n}_{\vec{\Psi}}$ its conformal factor, its mean curvature, its tracefree curvature and its Gauss map. Then $\vec{\Psi}_{z}=e^{i \omega} M_{-\omega} \vec{\Phi}_{z}^{1}\left(e^{i \omega}\right.$.) which implies $\vec{n}_{\vec{\Psi}}=M_{-\omega} \vec{n}$ and $e^{\lambda_{\vec{\Psi}}}=e^{\lambda^{1}}$. Consequently using 43.

$$
\vec{\Psi}_{z}(0)=\frac{e^{i \omega}}{2} M_{\omega}\left(\begin{array}{c}
1 \\
i \\
0
\end{array}\right)=\frac{e^{i \omega}}{2}\left(\begin{array}{c}
e^{-i \omega} \\
i e^{-i \omega} \\
0
\end{array}\right)=\frac{1}{2} \vec{e}_{0}
$$

Thus, one still has

$$
e^{\lambda \Psi}(0)=1
$$

. Moreover, we can compute $\vec{\Psi}_{z z}=e^{2 i \omega} M_{-\omega} \vec{\Phi}_{z z}^{1}\left(e^{i \omega}\right)$ and deduce

$$
\Omega_{\Psi}=e^{2 i \omega} \Omega^{1}
$$

which in turn implies

$$
\left[\Omega_{\Psi} e^{-\lambda_{\Psi}}\right](0)=e^{2 i \omega}\left[\Omega^{1} e^{-\lambda^{1}}\right](0) .
$$

Thanks to 290 , one can choose $\omega$ such that $e^{2 i \omega}=\frac{-2}{\left[\Omega^{1} e^{-\lambda^{1}}\right](0)}$. In that case $\Omega_{\Psi}(0) e^{-\lambda_{\Psi}(0)}=-2$, which yields thanks to 45

$$
\Omega_{\Psi}(0)=-2 .
$$

Comparing (29) to 46 , one can see that this adjustment done with rotations ensured a jump from an equality in moduli (namely (29) ) to an equality for complex numbers (that is (46)).

The sequence $\overrightarrow{\Psi^{\varepsilon}}=M_{\omega} \vec{\Phi}^{\varepsilon}\left(e^{i \omega}\right.$.) satisfies hypotheses 1,4 and 5 , while

$$
\begin{aligned}
& \vec{\Psi}^{\varepsilon} \rightarrow M_{\omega} \vec{\Phi}^{0}\left(e^{i \omega} .\right) \quad C_{\mathrm{loc}}^{\infty}(\mathbb{D} \backslash\{0\}), \\
& \widetilde{\Psi}^{\varepsilon} \rightarrow \Psi \quad C_{\mathrm{loc}}^{\infty}(\mathbb{C}) .
\end{aligned}
$$


We will not change notations for simplicity's sake, and will merely assume, without loss of generality, that

$$
\Omega^{1}(0)=-2
$$

\section{Summing up:}

$$
\begin{aligned}
& \vec{\Phi}_{z}^{1}(0)=\frac{1}{2} \vec{e}_{0}, \\
& \Omega^{1}(0)=-2, \\
& \left(\left|\Omega^{1}\right|^{2} e^{-2 \lambda^{1}}\right)_{z}(0)=0 .
\end{aligned}
$$

\section{Consequences on the Enneper-Weierstrass representation:}

Since $\vec{\Phi}^{1}$ is a minimal immersion we can use the Enneper-Weierstrass representation:

$$
\vec{\Phi}_{z}^{1}=\frac{f}{2}\left(\begin{array}{c}
1-g^{2} \\
i\left(1+g^{2}\right) \\
2 g
\end{array}\right)
$$

where $f$ is a holomorphic function on $\mathbb{C}$ and $g$ a meromorphic one. Since (according to (25)) $\vec{\Phi}^{1}$ has finite total curvature, $g$ is a meromorphic function of finite degree on $\mathbb{C}$. Thus there exists two polynomials $P, Q \in \mathbb{C}[X]$ such that $P \wedge Q=1$ and $g=\frac{P}{Q}$. Since $\vec{\Phi}^{1}$ has no end on $\mathbb{C}, f$ has a zero of order at least $2 k$ at each pole of order $k$ of $g$ (if not, 49 shows that at such a pole $\vec{\Phi}_{z} \rightarrow \infty$, that is: this point is an end of $\vec{\Phi}$ ). Consequently there exists a holomorphic function $\tilde{f}$ such that $f=Q^{2} \widetilde{f}$. In addition, $\vec{\Phi}^{1}$ has no branch point on $\mathbb{C}$ and one finite end at $\infty$, thus $\widetilde{f}$ is a holomorphic function without zeros and of finite order at infinity, i.e. a constant. We can then write

$$
\vec{\Phi}_{z}^{1}=\frac{1}{2}\left(\begin{array}{c}
Q^{2}-P^{2} \\
i\left(Q^{2}+P^{2}\right. \\
2 P Q
\end{array}\right)=\frac{Q^{2}}{2}\left(\begin{array}{l}
1 \\
i \\
0
\end{array}\right)-\frac{P^{2}}{2}\left(\begin{array}{c}
1 \\
-i \\
0
\end{array}\right)+P Q\left(\begin{array}{l}
0 \\
0 \\
1
\end{array}\right) .
$$

Further, since $\vec{\Phi}^{1}$ is assumed to have an end of multiplicity $\theta+1$ one can expand (50) as

$$
\vec{\Phi}_{z}^{1}=\widetilde{A} z^{\theta}+O\left(z^{\theta-1}\right)
$$

where $\widetilde{A} \in \mathbb{C}^{3} \backslash\{0\}$. Comparing (50) and 51) notably implies that $\theta$ is even and $P, Q \in \mathbb{C}_{\frac{\theta}{2}}[X]$. From (50) we then successively deduce

$$
\begin{gathered}
\vec{n}^{1}=\frac{1}{|P|^{2}+|Q|^{2}}\left(\begin{array}{c}
P \bar{Q}+\bar{P} Q \\
i(\bar{P} Q-P \bar{Q}) \\
|P|^{2}-|Q|^{2}
\end{array}\right) \\
e^{2 \lambda^{1}}=\left(|P|^{2}+|Q|^{2}\right)^{2} \\
\vec{\Phi}_{z z}^{1}=Q^{\prime}\left(\begin{array}{c}
Q \\
Q i \\
P
\end{array}\right)-P^{\prime}\left(\begin{array}{c}
P \\
-i P \\
-Q
\end{array}\right)
\end{gathered}
$$

which implies

$$
\Omega^{1}=2\left(P Q^{\prime}-P^{\prime} Q\right)
$$

and in turn

$$
\begin{gathered}
\Omega^{1} e^{-\lambda^{1}}=2 \frac{P Q^{\prime}-P^{\prime} Q}{|P|^{2}+|Q|^{2}}, \\
\left|\Omega^{1} e^{-\lambda^{1}}\right|^{2}=4 \frac{|P|^{2}\left|Q^{\prime}\right|^{2}+\left|P^{\prime}\right|^{2}|Q|^{2}-P \overline{P^{\prime}} Q^{\prime} \bar{Q}-P^{\prime} Q \overline{P Q^{\prime}}}{\left(|P|^{2}+|Q|^{2}\right)^{2}} .
\end{gathered}
$$

Comparing the first equality of $(48)$ to $(50)$ with $z=0$ ensures that $P(0)=0$ and $Q(0)=1$, while taking $(55)$ at $z=0$ and comparing it to the second equality of $\left(48\right.$ yields $P^{\prime}(0)=1$. Differentiating (57) at $z=0$ and comparing it to the third equality of 48 yields the last equality of 42 .

This concludes the proof. 


\subsection{Infinitesimal adjustments:}

This section will prove the infinitesimal counterpart of theorem 3.4

Lemma 3.5. Let $\vec{\Phi}^{\varepsilon}: \mathbb{D} \rightarrow \mathbb{R}^{3}$ be a sequence of Willmore conformal immersions satisfying hypotheses 1 1 6 . Up to infinitesimal adjustments we can assume that:

7 .

$$
\begin{aligned}
& \vec{\Phi}_{z}^{\varepsilon}(0)=\frac{C^{\varepsilon}}{\varepsilon} \vec{\Phi}_{z}^{1}(0)=\frac{C^{\varepsilon}}{2 \varepsilon} \vec{e}_{0}, \\
& {\left[\Omega^{\varepsilon} e^{-\lambda^{\varepsilon}}\right](0)=\frac{1}{\varepsilon}\left[\Omega^{1} e^{-\lambda^{1}}\right](0)=-\frac{2}{\varepsilon} .}
\end{aligned}
$$

\section{Proof. Using homothetic transformations of $\mathbb{R}^{3}$ :}

By hypothesis $3, \widetilde{\Phi}_{z}^{\varepsilon}(0) \rightarrow \vec{\Phi}_{z}^{1}(0)$, thus there exists a sequence of homothetic transformations $\sigma^{\varepsilon} \rightarrow I d$ such that $\sigma^{\varepsilon} \widetilde{\Phi}_{z}^{\varepsilon}(0)=\vec{\Phi}_{z}^{1}(0)$. Since $\sigma^{\varepsilon}$ tends toward the identity, hypotheses 1.3 are still satisfied, and hypothesis 5 still stands due to the conformal invariance properties of the tracefree curvature. We will then apply this sequence of transformations without changing the notations for simplicity's sake and assume $\widetilde{\Phi}_{z}^{\varepsilon}(0)=\vec{\Phi}_{z}^{1}(0)$. Considering

$$
\widetilde{\Phi}_{z}^{\varepsilon}=\frac{\varepsilon}{C^{\varepsilon}} \vec{\Phi}_{z}^{\varepsilon},
$$

we deduce with 48 ,

$$
\vec{\Phi}_{z}^{\varepsilon}(0)=\frac{C^{\varepsilon}}{2 \varepsilon} \vec{e}_{0}
$$

Adjusting the parametrization:

Using (29) and (27), we can set

$$
e^{2 i \omega^{\varepsilon}}=\frac{\left[\widetilde{\Omega}^{\varepsilon} e^{-\widetilde{\lambda}^{\varepsilon}}\right](0)}{\left[\Omega^{1} e^{-\lambda^{1}}\right](0)}=\varepsilon \frac{\left[\Omega^{\varepsilon} e^{-\lambda^{\varepsilon}}\right](0)}{\left[\Omega^{1} e^{-\lambda^{1}}\right](0)} \rightarrow 1 .
$$

We consider $\vec{\Psi}^{\varepsilon}=M_{\omega^{\varepsilon}} \vec{\Phi}^{\varepsilon}\left(e^{i \omega^{\varepsilon}}\right.$.). Since $e^{i \omega^{\varepsilon}} \rightarrow 1, \vec{\Psi}^{\varepsilon}$ satisfies $1.6[$ [ 5 is still satisfied due to the invariance properties of the tracefree curvature). As detailed in the previous section we have

$$
\widetilde{\Omega}_{\Psi}^{\varepsilon} e^{-\widetilde{\lambda}_{\Psi}^{\varepsilon}}=-2,
$$

which implies

$$
\left[\Omega_{\Psi}^{\varepsilon} e^{-\lambda_{\Psi}^{\varepsilon}}\right](0)=-\frac{2}{\varepsilon}
$$

For simplicity's sake we will not change the notations and assume that $\vec{\Phi}^{\varepsilon}$ satisfies

$$
\left[\Omega^{\varepsilon} e^{-\lambda^{\varepsilon}}\right](0)=-\frac{2}{\varepsilon}
$$

This gives us the desired result.

\section{Expanding the conformal factor}

This section will prove the following expansion on the conformal factor, which will serve as a stepping-stone in the proof of theorem 1.5 .

Theorem 4.1. Let $\vec{\Phi}^{\varepsilon}$ be a sequence of Willmore conformal immersions satisfying 1.7 . Then there exists $l^{\varepsilon} \in L^{\infty}(\mathbb{D})$ such that:

$$
\begin{aligned}
& \lambda^{\varepsilon}=\ln \left(\varepsilon^{\theta}+r^{\theta}\right)+l^{\varepsilon}, \\
& \left\|l^{\varepsilon}\right\|_{L^{\infty}(\mathbb{D})} \leq C\left(C_{0}\right) .
\end{aligned}
$$

As a result if we denote $\chi=\sqrt{\varepsilon^{2}+r^{2}}$, the immersion satisfies the following Harnack inequality:

$$
\frac{\chi^{\theta}}{C\left(C_{0}\right)} \leq e^{\lambda^{\varepsilon}} \leq C\left(C_{0}\right) \chi^{\theta} .
$$


Proof. Step 1: Controls in the neck area

Given hypothesis 4 , for any $\varepsilon_{0}>0$ arbitrarily small there exists $R$ big enough such that

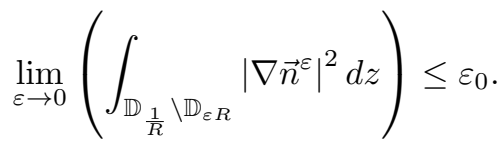

We first recall lemma V.3 of [4.

Lemma 4.2. There exists a constant $\eta>0$ with the following property. Let $0<4 r<R<\infty$. If $\vec{\Phi}$ is any (weak) conformal immersion of $\Omega:=\mathbb{D}_{R} \backslash \mathbb{D}_{r}$ into $\mathbb{R}^{3}$ with $L^{2}$-bounded second fundamental form and satisfying

$$
\|\nabla \vec{n}\|_{L^{2, \infty}(\Omega)}<\sqrt{\eta},
$$

then there exist $\frac{1}{2}<\alpha<1$ and $A \in \mathbb{R}$ depending on $R, r, m$ and $\vec{\Phi}$ such that

$$
\|\lambda(x)-d \ln |x|-A\|_{L^{\infty}\left(\mathbb{D}_{\alpha R} \backslash \mathbb{D}_{\frac{r}{\alpha}}\right)} \leq C\left(\|\nabla \lambda\|_{L^{2, \infty}(\Omega)}+\int_{\Omega}|\nabla \vec{n}|^{2}\right),
$$

where $d$ satisfies

$$
\begin{aligned}
\mid 2 \pi d-\int_{\partial \mathbb{D}_{r}} \partial_{r} \lambda d l_{\partial \mathbb{D}_{r} \mid \leq} & {\left[\int_{\mathbb{D}_{2 r} \backslash \mathbb{D}_{r}}|\nabla \vec{n}|^{2} d z\right.} \\
& \left.+\frac{1}{\ln \frac{R}{r}}\left(\|\nabla \lambda\|_{L^{2, \infty}(\Omega)}+\int_{\Omega}|\nabla \vec{n}|^{2}\right)\right] .
\end{aligned}
$$

Thus, according to $(60)$, there exists $R_{0}$ such that for all $R \geq R_{0}$ and $\varepsilon$ small enough, we can apply lemma 4.2 on $\mathbb{D}_{\frac{1}{R}} \backslash \mathbb{D}_{\varepsilon R}$ and conclude that there exists $d_{R}^{\varepsilon}$ and $A_{R}^{\varepsilon} \in \mathbb{R}$ such that

$$
\begin{aligned}
& \left\|\lambda^{\varepsilon}(x)-d_{R}^{\varepsilon} \ln r-A_{R}^{\varepsilon}\right\|_{L^{\infty}\left(\mathbb{D}_{\frac{1}{2 R}} \backslash \mathbb{D}_{2 \varepsilon R}\right)} \leq C_{0}, \\
& \left|d_{R}^{\varepsilon}-\frac{1}{2 \pi} \int_{\partial \mathbb{D}_{\varepsilon R}} \partial_{r} \lambda^{\varepsilon} d l_{\partial \mathbb{D}_{\varepsilon R}}\right| \leq C\left[\int_{\mathbb{D}_{2 \varepsilon R} \backslash \mathbb{D}_{\varepsilon R}}|\nabla \vec{n}|^{2} d z+\frac{C_{0}}{-\ln \left(\varepsilon R^{2}\right)}\right] .
\end{aligned}
$$

Here $C_{0}$ is the uniform bound given by hypothesis 1 (up to a multiplicative uniform constant). We saw in 41. that

$$
\lim _{R \rightarrow \infty}\left(\lim _{\varepsilon \rightarrow 0} \frac{1}{2 \pi} \int_{\partial \mathbb{D}_{\varepsilon R}} \partial_{r} \lambda^{\varepsilon} d l_{\partial \mathbb{D}_{\varepsilon R}}\right)=\theta
$$

while hypothesis 4 ensures that

$$
\lim _{R \rightarrow \infty}\left(\lim _{\varepsilon \rightarrow 0} \int_{\mathbb{D}_{2 \varepsilon R} \backslash \mathbb{D}_{\varepsilon R}}|\nabla \vec{n}|^{2} d z\right)=0 .
$$

Hence we can fix $R_{1}>0$ such that for $\varepsilon$ small enough:

$$
\left|d^{\varepsilon}-\theta\right| \leq \frac{1}{10^{3}} .
$$

Since $R_{1}$ is fixed, we will get rid of the subscript on $d^{\varepsilon}$ and $A^{\varepsilon}$. Then for any $\varepsilon$ small enough:

$$
\left\|\lambda^{\varepsilon}(x)-d^{\varepsilon} \ln r-A^{\varepsilon}\right\|_{L^{\infty}\left(\mathbb{D}_{\frac{1}{2 R_{1}}} \backslash \mathbb{D}_{2 \varepsilon R_{1}}\right)} \leq C\left(C_{0}, R_{1}\right) .
$$

\section{Step 2: Estimates on the exterior boundary:}

Hypothesis 2 ensures that on $\partial \mathbb{D}_{\frac{1}{2 R_{1}}}, \lambda^{\varepsilon} \rightarrow \lambda^{0}$ smoothly, and that $\lambda^{0}$ is a bounded function away from 0 , which implies

$$
\left\|\lambda^{\varepsilon}\right\|_{L^{\infty}\left(\partial \mathbb{D} \frac{1}{2 R_{1}}\right)} \leq C\left(C_{0}, R_{1}\right)
$$

On the other hand $(65)$ ensures

$$
\left|d^{\varepsilon} \ln R_{1}\right| \leq C\left(C_{0}, R_{1}\right) .
$$


As a result, combining 66 on $\partial \mathbb{D}_{\frac{1}{2 R_{1}}}, 67$, and 68 yields

$$
\left|A^{\varepsilon}\right| \leq C\left(C_{0}, R_{1}\right)
$$

which we can inject in (66) to obtain

$$
\left\|\lambda^{\varepsilon}(x)-d^{\varepsilon} \ln r\right\|_{L^{\infty}\left(\mathbb{D}_{\frac{1}{2 R_{1}}} \backslash \mathbb{D}_{2 \varepsilon R_{1}}\right)} \leq C\left(C_{0}, R_{1}\right) .
$$

Step 3: Estimates on the interior boundary:

Estimate 696) implies

$$
\left\|\lambda^{\varepsilon}(x)-d^{\varepsilon} \ln r\right\|_{L^{\infty}\left(\partial \mathbb{D}_{2 \varepsilon R_{1}}\right)} \leq C\left(C_{0}, R_{1}\right) .
$$

In addition, 260 yields

$$
\left\|\lambda^{\varepsilon}(x)-d^{\varepsilon} \ln r\right\|_{L^{\infty}\left(\partial \mathbb{D}_{2 \varepsilon R_{1}}\right)}=\left\|\tilde{\lambda}^{\varepsilon}(x)-d^{\varepsilon} \ln r+\ln \left(\frac{C^{\varepsilon}}{\varepsilon}\right)-d^{\varepsilon} \ln \varepsilon\right\|_{L^{\infty}\left(\partial \mathbb{D}_{2 R_{1}}\right)} .
$$

Hypothesis 3 then ensures that

$$
\left\|\widetilde{\lambda}^{\varepsilon}\right\|_{L^{\infty}\left(\mathbb{D}_{2 R_{1}}\right)} \leq C\left(C_{0}, R_{1}\right)
$$

and 65 that

$$
\left\|d^{\varepsilon} \ln r\right\|_{L^{\infty}\left(\mathbb{D}_{2 R_{1}}\right)} \leq C\left(C_{0}, R_{1}\right)
$$

Together (70), 71) and (72) yield

$$
\left|\ln \left(\frac{C^{\varepsilon}}{\varepsilon^{d^{\varepsilon}+1}}\right)\right| \leq C\left(C_{0}, R_{1}\right) .
$$

A direct consequence of 65 and 73 is the following estimate:

$$
\frac{\varepsilon^{\theta+1-10^{-3}}}{C\left(R_{1}, C_{0}\right)} \leq \frac{\varepsilon^{d^{\varepsilon}+1}}{C\left(C_{0}, R_{1}\right)} \leq C^{\varepsilon} \leq C\left(C_{0}, R_{1}\right) \varepsilon^{d^{\varepsilon}+1} \leq C\left(C_{0}, R_{1}\right) \varepsilon^{\theta+1+10^{-3}} .
$$

Step 4: Expanding the conformal factor on the whole disk:

We forcefully write $\lambda^{\varepsilon}=\ln \left(\varepsilon^{d^{\varepsilon}}+r^{d^{\varepsilon}}\right)+l^{\varepsilon}$. We aim to show that

$$
\left\|l^{\varepsilon}\right\|_{L^{\infty}(\mathbb{D})} \leq C\left(C_{0}, R_{1}\right) .
$$

On $\mathbb{D} \backslash \mathbb{D}_{\frac{1}{4 R_{1}}}$ :

Using hypothesis 2 ,

$$
\left\|\lambda^{\varepsilon}\right\|_{L^{\infty}\left(\mathbb{D} \backslash \mathbb{D}_{\frac{1}{4 R_{1}}}\right)} \leq C\left(C_{0}, R_{1}\right) .
$$

One might also notice that, thanks to 65 , on $\mathbb{D} \backslash \mathbb{D}_{\frac{1}{4 R_{1}}}$ :

$$
\left|\ln \left(\varepsilon^{d^{\varepsilon}}+r^{d^{\varepsilon}}\right)\right| \leq C\left(C_{0}, R_{1}\right)\left|\ln \left(\left(\frac{1}{2 R_{1}}\right)^{m+\frac{1}{100}}\right)\right| .
$$

Then using (75) and (76):

$$
\begin{aligned}
\left.\left\|l^{\varepsilon}\right\|_{L^{\infty}\left(\mathbb{D} \backslash \mathbb{D} \frac{1}{4 R_{1}}\right.}\right) & \leq\left\|\lambda^{\varepsilon}\right\|_{L^{\infty}\left(\mathbb{D} \backslash \mathbb{D}_{\frac{1}{4 R_{1}}}\right)}+\left\|\ln \left(\varepsilon^{d^{\varepsilon}}+r^{d^{\varepsilon}}\right)\right\|_{L^{\infty}\left(\mathbb{D} \backslash \mathbb{D} \frac{1}{4 R_{1}}\right)} \\
& \leq C\left(C_{0}, R_{1}\right) .
\end{aligned}
$$

On $\mathbb{D}_{4 \varepsilon R_{1}}$ :

Using hypothesis 3

$$
\left\|\widetilde{\lambda}^{\varepsilon}\right\|_{L^{\infty}\left(\mathbb{D}_{4 R_{1}}\right)} \leq C\left(C_{0}, R_{1}\right)
$$


while thanks to 73

$$
\begin{aligned}
\left\|\ln \left(\varepsilon^{d^{\varepsilon}}+r^{d^{\varepsilon}}\right)-\ln \left(\frac{C^{\varepsilon}}{\varepsilon}\right)\right\|_{L^{\infty}\left(\mathbb{D}_{4 \varepsilon R_{1}}\right)} & \leq\left\|\ln \left(\varepsilon^{d^{\varepsilon}}+(\varepsilon r)^{d^{\varepsilon}}\right)-\ln \left(\frac{C^{\varepsilon}}{\varepsilon}\right)\right\|_{L^{\infty}\left(\mathbb{D}_{4 R_{1}}\right)} \\
& \leq\left\|\ln \left(1+r^{d^{\varepsilon}}\right)\right\|_{L^{\infty}\left(\mathbb{D}_{4 R_{1}}\right)}+\left\|\ln \left(\frac{C^{\varepsilon}}{\varepsilon^{d^{\varepsilon}+1}}\right)\right\|_{L^{\infty}\left(\mathbb{D}_{4 R_{1}}\right)} \\
& \leq C\left(C_{0}, R_{1}\right) .
\end{aligned}
$$

To conclude we deduce thanks to 78 and 79 :

$$
\begin{aligned}
\left\|l^{\varepsilon}\right\|_{L^{\infty}\left(\mathbb{D}_{4 \varepsilon R_{1}}\right)} & \leq\left\|\lambda^{\varepsilon}-\ln \left(\varepsilon^{d^{\varepsilon}}+r^{d^{\varepsilon}}\right)+\ln \left(\frac{C^{\varepsilon}}{\varepsilon}\right)-\ln \left(\frac{C^{\varepsilon}}{\varepsilon}\right)\right\|_{L^{\infty}\left(\mathbb{D}_{4 \varepsilon} R_{1}\right)} \\
& \leq\left\|\lambda^{\varepsilon}-\ln \left(\frac{C^{\varepsilon}}{\varepsilon}\right)\right\|_{L^{\infty}\left(\mathbb{D}_{4 \varepsilon R_{1}}\right)}+\left\|\ln \left(\varepsilon^{d^{\varepsilon}}+r^{d^{\varepsilon}}\right)-\ln \left(\frac{C^{\varepsilon}}{\varepsilon}\right)\right\|_{L^{\infty}\left(\mathbb{D}_{4 \varepsilon R_{1}}\right)} \\
& \leq\left\|\tilde{\lambda}^{\varepsilon}\right\|_{L^{\infty}\left(\mathbb{D}_{4 R_{1}}\right)}+\left\|\ln \left(\varepsilon^{d^{\varepsilon}}+r^{d^{\varepsilon}}\right)-\ln \left(\frac{C^{\varepsilon}}{\varepsilon}\right)\right\|_{L^{\infty}\left(\mathbb{D}_{4 \varepsilon R_{1}}\right)} \\
& \leq C\left(C_{0}, R_{1}\right) .
\end{aligned}
$$

On $\mathbb{D}_{\frac{1}{2 R_{1}}} \backslash \mathbb{D}_{2 \varepsilon R_{1}}$ :

Thanks to (69):

$$
\begin{aligned}
\left\|l^{\varepsilon}\right\|_{L^{\infty}\left(\mathbb{D}_{\frac{1}{2 R_{1}}} \backslash \mathbb{D}_{2 \varepsilon R_{1}}\right)} & \leq\left\|\lambda^{\varepsilon}-d^{\varepsilon} \ln r\right\|_{L^{\infty}\left(\mathbb{D}_{\frac{1}{2 R_{1}}} \backslash \mathbb{D}_{2 \varepsilon R_{1}}\right)}+\left\|\ln \left(\frac{r^{d^{\varepsilon}}}{\varepsilon^{d^{\varepsilon}}+r^{d^{\varepsilon}}}\right)\right\|_{L^{\infty}\left(\mathbb{D}_{\frac{1}{2 R_{1}}} \backslash \mathbb{D}_{2 \varepsilon R_{1}}\right)} \\
& \leq C\left(C_{0}, R_{1}\right) .
\end{aligned}
$$

Combining (77), 80 and 81 yields

$$
\left\|l^{\varepsilon}\right\|_{L^{\infty}(\mathbb{D})} \leq C\left(C_{0}, R_{1}\right),
$$

which is as desired. We now wish to refine this first expansion by showing that $d^{\varepsilon}$ converges toward $\theta$ fast enough to be updated in 696.

\section{Step 5: Refinement:}

A consequence of estimate 82 is the following Harnack inequality on the conformal factor:

$$
\frac{\varepsilon^{d^{\varepsilon}}+r^{d^{\varepsilon}}}{C\left(C_{0}, R_{1}\right)} \leq e^{\lambda^{\varepsilon}} \leq C\left(C_{0}, R_{1}\right)\left(\varepsilon^{d^{\varepsilon}}+r^{d^{\varepsilon}}\right)
$$

which, using the notation $\chi=\sqrt{\varepsilon^{2}+r^{2}}$, we will rewrite in the more convenient form

$$
\frac{\chi^{d^{\varepsilon}}}{C\left(C_{0}, R_{1}\right)} \leq e^{\lambda^{\varepsilon}} \leq C\left(C_{0}, R_{1}\right) \chi^{d^{\varepsilon}}
$$

Injecting $(65)$ into $(83)$ yields

$$
e^{\lambda^{\varepsilon}} \leq C\left(C_{0}, R_{1}\right) \chi^{\theta-10^{-3}}
$$

Since $\vec{\Phi}^{\varepsilon}$ is conformal,

$$
\Delta \vec{\Phi}^{\varepsilon}=2 H^{\varepsilon} e^{2 \lambda^{\varepsilon}} \vec{n}^{\varepsilon}=\chi^{\theta-10^{-3}} 2 H^{\varepsilon} e^{\lambda^{\varepsilon}} \frac{e^{\lambda^{\varepsilon}}}{\chi^{\theta-10^{-3}}} \vec{n}^{\varepsilon} .
$$

Noticing that 355 and 84 imply

$$
\left\|2 H^{\varepsilon} e^{\lambda^{\varepsilon}} \frac{e^{\lambda^{\varepsilon}}}{\chi^{\theta-10^{-3}}} \vec{n}^{\varepsilon}\right\|_{L^{\infty}(\mathbb{D})} \leq C\left(C_{0}, R_{1}\right),
$$

we can apply theorem A.3 (see in the appendix) to equation 85 and find:

$$
\vec{\Phi}_{z}^{\varepsilon}=\vec{P}^{\varepsilon}(z)+\vec{\varphi}_{0}^{\varepsilon},
$$


where $\vec{P}^{\varepsilon}=\sum_{q=0}^{\theta} \vec{p}_{q}^{\varepsilon} z^{q} \in \mathbb{C}_{\theta}[X]$ with $\left|\vec{p}_{q}^{\varepsilon}\right| \leq C\left(C_{0}, R_{1}\right)$ for all $q \leq \theta$ and $\vec{\varphi}_{0}^{\varepsilon}: \mathbb{D} \rightarrow \mathbb{R}^{3}$ satisfies

$$
\begin{aligned}
& \forall \kappa>0 \quad\left\|\frac{\vec{\varphi}_{0}^{\varepsilon}}{\chi^{\theta+1-10^{-3}-\kappa}}\right\|_{L^{\infty}(\mathbb{D})} \leq C_{\kappa}\left(C_{0}, R_{1}\right), \\
& \forall p<\infty \quad\left\|\frac{\nabla \vec{\varphi}_{0}^{\varepsilon}}{\chi^{\theta-10^{-3}}}\right\|_{L^{p}(\mathbb{D})} \leq C_{p}\left(C_{0}, R_{1}\right) .
\end{aligned}
$$

By convergence of $\vec{\Phi}^{\varepsilon}$ away from zero (hypothesis 27, $\vec{p}_{q}^{\varepsilon} \rightarrow \vec{p}_{q} \in \mathbb{C}$ as $\varepsilon$ goes to 0 . Furthermore, 88) yields $\vec{\varphi}_{0}^{\varepsilon} \rightarrow \vec{\varphi}_{0} W^{1, p}(\mathbb{D})$, with $\varphi_{0}$ satisfying

$$
\begin{aligned}
& \forall \kappa>0 \quad\left\|\frac{\vec{\varphi}_{0}}{r^{\theta+1-10^{-3}-\kappa}}\right\|_{L^{\infty}(\mathbb{D})} \leq C_{\kappa}\left(C_{0}, R_{1}\right), \\
& \forall p<\infty \quad\left\|\frac{\nabla \overrightarrow{\varphi_{0}}}{r^{\theta-10^{-3}}}\right\|_{L^{p}(\mathbb{D})} \leq C_{p}\left(C_{0}, R_{1}\right),
\end{aligned}
$$

since $\chi \rightarrow r$ as $\varepsilon \rightarrow 0$. Then 87 ensures that

$$
\vec{\Phi}_{z}^{\varepsilon} \rightarrow \sum_{q=0}^{m} \vec{p}_{q} z^{q}+\vec{\varphi}_{0} .
$$

Since we assumed that $\vec{\Phi}^{\varepsilon} \rightarrow \vec{\Phi}^{0}$ away from 0, comparing $(32)$ and $(90)$ yields

$$
\begin{aligned}
& \forall q<\theta \quad \vec{p}_{q}^{\varepsilon} \rightarrow 0 \\
& \vec{p}_{\theta}^{\varepsilon} \rightarrow \vec{A} \neq 0 .
\end{aligned}
$$

Besides, 877) gives the following

$$
\widetilde{\Phi}_{z}^{\varepsilon}=\sum_{q=0}^{\theta} \vec{p}_{q}^{\varepsilon} \frac{\varepsilon^{q+1}}{C^{\varepsilon}} z^{q}+\frac{\varepsilon \vec{\varphi}_{0}^{\varepsilon}(\varepsilon \cdot)}{C^{\varepsilon}} .
$$

One might also notice using 88

$$
\begin{aligned}
\left|\vec{\varphi}_{0}^{\varepsilon}\right|(\varepsilon z) & \leq C\left(C_{0}, R_{1}\right) \chi^{\theta+\frac{1}{2}}(\varepsilon z) \\
& \leq \varepsilon^{\theta+\frac{1}{2}} C\left(C_{0}, R_{1}\right){\sqrt{1+r^{2}}}^{\theta+\frac{1}{2}} .
\end{aligned}
$$

This, along with (73), implies

$$
\begin{aligned}
\left|\frac{\varepsilon \vec{\varphi}_{0}^{\varepsilon}(\varepsilon .)}{C^{\varepsilon}}\right| & \leq\left|\frac{\varepsilon^{\theta+1+\frac{1}{2}}}{C^{\varepsilon}}\right| C\left(C_{0}, R_{1}\right){\sqrt{1+r^{2}}}^{\theta+\frac{1}{2}} \\
& \leq C\left(C_{0}, R_{1}\right){\sqrt{1+r^{2}}}^{\theta+\frac{1}{2}} \varepsilon^{\frac{1}{2}} .
\end{aligned}
$$

Consequently

$$
\frac{\varepsilon \vec{\varphi}_{0}^{\varepsilon}(\varepsilon z)}{C^{\varepsilon}} \rightarrow 0 \quad L_{\text {loc }}^{\infty}(\mathbb{C}) .
$$

Since $\widetilde{\Phi}^{\varepsilon}$ is assumed to converge smoothly towards $\vec{\Phi}^{1}$ on compacts of $\mathbb{C}$, we deduce from (51), 92 and (94)

$$
\sum_{q=0}^{\theta} \vec{p}_{q}^{\varepsilon} \frac{\varepsilon^{q+1}}{C^{\varepsilon}} z^{q} \rightarrow \vec{\Phi}_{z}^{1}=\widetilde{A} z^{\theta}+O\left(z^{\theta-1}\right) .
$$

Hence

$$
\frac{\varepsilon^{\theta+1}}{C^{\varepsilon}} \vec{p}_{\theta}^{\varepsilon} \rightarrow \widetilde{A} \neq 0 .
$$

Further, given that $p_{\theta}^{\varepsilon} \rightarrow \vec{A} \neq 0$, there exists $C\left(C_{0}, R_{1}\right)>0$ such that

$$
\left|\ln \left(\frac{C^{\varepsilon}}{\varepsilon^{\theta+1}}\right)\right| \leq C\left(C_{0}, R_{1}\right) \text {. }
$$


Combining (73) and (96) yields

$$
\left|\ln \left(\frac{\varepsilon^{d^{\varepsilon}}}{\varepsilon^{\theta}}\right)\right| \leq C\left(C_{0}, R_{1}\right)
$$

which ensures

$$
\left|\left(d^{\varepsilon}-\theta\right) \ln \varepsilon\right| \leq C\left(C_{0}, R_{1}\right) .
$$

Then, 69) and (97) combine and yield

$$
\begin{aligned}
\left\|\lambda^{\varepsilon}-\theta \ln r\right\|_{L^{\infty}\left(\mathbb{D}_{\frac{1}{2 R_{1}}} \backslash \mathbb{D}_{2 \varepsilon R_{1}}\right)} & \leq\left\|\lambda^{\varepsilon}-\theta \ln r\right\|_{L^{\infty}\left(\mathbb{D}_{\frac{1}{2 R_{1}}} \backslash \mathbb{D}_{2 \varepsilon R_{1}}\right)}+\left\|\left(\theta-d^{\varepsilon}\right) \ln r\right\|_{L^{\infty}\left(\mathbb{D}_{\frac{1}{2 R_{1}}} \backslash \mathbb{D}_{2 \varepsilon R_{1}}\right)} \\
& \leq C\left(C_{0}, R_{1}\right) .
\end{aligned}
$$

Since inequality $(98)$ is analogous to $(69)$, we can do all the reasonings from 69$)$ to $(95)$ with $d^{\varepsilon}=\theta$. Then the conformal factor satisfies:

$$
\lambda^{\varepsilon}=\ln \left(\varepsilon^{\theta}+r^{\theta}\right)+l^{\varepsilon}
$$

with $l^{\varepsilon}$ such that

$$
\left\|l^{\varepsilon}\right\|_{L^{\infty}(\mathbb{D})} \leq C\left(C_{0}, R_{1}\right)
$$

and

$$
\frac{\chi^{\theta}}{C\left(C_{0}, R_{1}\right)} \leq e^{\lambda^{\varepsilon}} \leq C\left(C_{0}, R_{1}\right) \chi^{\theta} .
$$

This concludes the proof of the desired result since $R_{1}$ is fixed.

Moreover, for simplicity's sake we can, up to an inconsequential (thanks to (96)) adjustment, assume $C^{\varepsilon}=\varepsilon^{\theta+1}$. Then, exploiting (95) yields:

$$
\widetilde{A}=\vec{A}
$$

$$
\sum_{q=0}^{\theta} \vec{p}_{q}^{\varepsilon} \varepsilon^{q-\theta} z^{q} \rightarrow \vec{\Phi}_{z}^{1}
$$

We can then decompose

$$
\vec{P}^{\varepsilon}=\varepsilon^{\theta} \vec{\Phi}_{z}^{1}\left(\frac{z}{\varepsilon}\right)+\varepsilon^{\theta} Q^{\varepsilon}\left(\frac{z}{\varepsilon}\right),
$$

with $\vec{Q}^{\varepsilon} \in \mathbb{C}_{\theta}[X]$ such that $\vec{Q}^{\varepsilon} \rightarrow 0$.

$\vec{\Phi}^{\varepsilon}$ then satisfies the following decomposition:

$$
\vec{\Phi}_{z}^{\varepsilon}=\varepsilon^{\theta} \vec{\Phi}_{z}^{1}\left(\frac{z}{\varepsilon}\right)+\varepsilon^{\theta} \vec{Q}\left(\frac{z}{\varepsilon}\right)+\vec{\varphi}_{0}^{\varepsilon},
$$

where

$$
\begin{aligned}
& \forall \kappa>0 \quad\left\|\frac{\vec{\varphi}_{0}^{\varepsilon}}{\chi^{\theta+1-\kappa}}\right\|_{L^{\infty}(\mathbb{D})} \leq C_{\kappa}\left(C_{0}, R_{1}\right), \\
& \forall p<\infty \quad\left\|\frac{\nabla \vec{\varphi}_{0}^{\varepsilon}}{\chi^{\theta}}\right\|_{L^{p}(\mathbb{D})} \leq C_{p}\left(C_{0}, R_{1}\right) .
\end{aligned}
$$

Remark 4.2.1. Equality 101 can be seen as prolonging theorem 3.2 not only must the multiplicity of the end and the multiplicity of the branch point correspond, but so must the parametrization of the limit planes in both cases.

Remark 4.2.2. A. Michelat and T. Rivière have presented the author with another proof of the expansion which works in the more general framework of any simple bubble (in [21]).

\section{Conditions on the limit surface:}

The aim of this section is to prove both theorem 1.5 and corollary 1.6 


\subsection{Proof of theorem $\mathbf{1 . 5}$}

As explained in section 3 we can equivalently work in conformal parametrizations under hypotheses 10 . We will then instead prove:

Theorem 5.1. Let $\vec{\Phi}^{\varepsilon}: \mathbb{D} \rightarrow \mathbb{R}^{3}$ be a sequence of Willmore conformal immersions satisfying hypotheses 1.7 . Then the second residue of $\vec{\Phi}^{0}$ at 0 satisfies

$$
\alpha \leq \theta-1
$$

\section{Proof. Step 1: Expansion of $\vec{\Phi}_{z z}^{\varepsilon}$ :}

We consider $\vec{\Phi}^{\varepsilon}$ satisfying hypotheses 1] 7, and thus (99)-(103). The system (7) of [19] states

$$
\left\{\begin{array}{l}
\Delta S^{\varepsilon}=\left\langle H^{\varepsilon} \nabla \vec{\Phi}^{\varepsilon}, \nabla^{\perp} \vec{R}^{\varepsilon}\right\rangle \\
\Delta \vec{R}^{\varepsilon}=-H^{\varepsilon} \nabla \vec{\Phi}^{\varepsilon} \times \nabla^{\perp} \vec{R}^{\varepsilon}-\nabla^{\perp} S^{\varepsilon} H^{\varepsilon} \nabla \vec{\Phi}^{\varepsilon} \\
\Delta \vec{\Phi}^{\varepsilon}=\frac{1}{2}\left(\nabla^{\perp} S^{\varepsilon} \cdot \nabla \vec{\Phi}^{\varepsilon}+\nabla^{\perp} \vec{R}^{\varepsilon} \times \nabla \vec{\Phi}^{\varepsilon}\right) .
\end{array}\right.
$$

Then (35), 37) and (104) yield:

$$
\left\|\Delta S^{\varepsilon}\right\|_{L^{\infty}(\mathbb{D})}+\left\|\Delta \vec{R}^{\varepsilon}\right\|_{L^{\infty}(\mathbb{D})} \leq C\left(C_{0}\right)
$$

Applying theorem A.3 (in the appendix) gives the following decomposition on $S^{\varepsilon}$ and $\vec{R}^{\varepsilon}$ :

$$
\begin{aligned}
S_{z}^{\varepsilon} & =S_{z}^{\varepsilon}(0)+\sigma_{0}^{\varepsilon} \\
\vec{R}_{z}^{\varepsilon} & =\vec{R}_{z}^{\varepsilon}(0)+\vec{\rho}_{0}^{\varepsilon},
\end{aligned}
$$

with $\sigma_{0}^{\varepsilon}$ and $\rho_{0}^{\varepsilon}$ satisfying

$$
\begin{aligned}
& \forall \kappa>0 \quad\left|\sigma_{0}^{\varepsilon}\right|(z)+\left|\bar{\rho}_{0}^{\varepsilon}\right|(z) \leq C_{\kappa}\left(C_{0}\right) \chi^{1-\kappa}, \\
& \forall p<\infty \quad\left\|\nabla \sigma_{0}^{\varepsilon}\right\|_{L^{p}(\mathbb{D})}+\left\|\nabla \bar{\rho}_{0}^{\varepsilon}\right\|_{L^{p}(\mathbb{D})} \leq C_{p}\left(C_{0}\right) .
\end{aligned}
$$

Injecting 102 and 106 into the third equation of 104 yields

$$
\Delta \vec{\Phi}^{\varepsilon}=2 \Im\left(\vec{R}_{z}^{\varepsilon}(0) \times \overline{\left[\varepsilon^{\theta} \vec{\Phi}_{z}^{1}\left(\frac{z}{\varepsilon}\right)+\varepsilon^{\theta} \vec{Q}^{\varepsilon}\left(\frac{z}{\varepsilon}\right)\right]}+S_{z}^{\varepsilon}(0) \overline{\left[\varepsilon^{\theta} \vec{\Phi}_{z}^{1}\left(\frac{z}{\varepsilon}\right)+\varepsilon^{\theta} \vec{Q}^{\varepsilon}\left(\frac{z}{\varepsilon}\right)\right]}\right)+\vec{\Psi}_{0}^{\varepsilon},
$$

where

$$
\vec{\Psi}_{0}^{\varepsilon}:=2 \Im\left(\vec{\rho}_{0}^{\varepsilon} \times \overline{\vec{\Phi}_{z}^{\varepsilon}}+\sigma_{0}^{\varepsilon} \overline{\vec{\Phi}_{z}^{\varepsilon}}+\vec{R}_{z}^{\varepsilon} \times \overline{\vec{\varphi}_{0}^{\varepsilon}}+S_{z}^{\varepsilon} \overline{\vec{\varphi}_{0}^{\varepsilon}}\right)
$$

satisfies

$$
\begin{array}{ll}
\forall \kappa>0 & \left|\vec{\Psi}_{0}^{\varepsilon}\right|(z) \leq C_{\kappa}\left(C_{0}\right) \chi^{\theta+1-\kappa}, \\
\forall p<\infty & \left\|\frac{\nabla \vec{\Psi}_{0}^{\varepsilon}}{\chi^{\theta}}\right\|_{L^{p}(\mathbb{D})} \leq C_{p}\left(C_{0}\right) .
\end{array}
$$

One may notice that

$$
\widetilde{h}^{\varepsilon}:=2 \Im\left(\vec{R}_{z}^{\varepsilon}(0) \times \overline{\left[\varepsilon^{\theta} \vec{\Phi}_{z}^{1}\left(\frac{z}{\varepsilon}\right)+\varepsilon^{\theta} \vec{Q}^{\varepsilon}\left(\frac{z}{\varepsilon}\right)\right]}+S_{z}^{\varepsilon}(0) \overline{\left[\varepsilon^{\theta} \vec{\Phi}_{z}^{1}\left(\frac{z}{\varepsilon}\right)+\varepsilon^{\theta} \vec{Q}^{\varepsilon}\left(\frac{z}{\varepsilon}\right)\right]}\right)
$$

is the sum of a polynomial of degree $\theta$ in $z$ and a polynomial of degree $\theta$ in $\bar{z}$, whose coefficients are uniformly bounded by a constant depending on $C_{0}$. Additionally it is of order $O\left(\chi^{\theta}\right)$ thanks to theorem 4.1. We can then find a polynomial $\vec{h}^{\varepsilon}$ in $z$ and $\bar{z}$ of total degree $\theta+2$ such that

$$
\begin{aligned}
& \vec{h}^{\varepsilon}(0)=\vec{h}_{z}^{\varepsilon}(0)=\vec{h}_{z}^{\varepsilon}(0)=0, \vec{h}^{\varepsilon}=O\left(\chi^{\theta+2}\right) \\
& \Delta \vec{h}^{\varepsilon}=\widetilde{h}^{\varepsilon} .
\end{aligned}
$$

Then

$$
\Delta\left(\vec{\Phi}^{\varepsilon}-\vec{h}^{\varepsilon}\right)=\Psi_{0}^{\varepsilon}
$$


Applying theorem A.8 (in the appendix) to 110, with $a=\theta+1-\kappa$ for $\kappa$ arbitrarily small yields

$$
\vec{\Phi}_{z}^{\varepsilon}=\vec{P}^{\varepsilon}(z)+\vec{h}_{z}^{\varepsilon}+\vec{\varphi}_{1}^{\varepsilon},
$$

where $P^{\varepsilon}$ is a polynomial of degree $\theta+1$ that we can split $\vec{P}^{\varepsilon}=\vec{P}_{\theta}^{\varepsilon}+\vec{p}^{\varepsilon} z^{\theta+1}$ with $\vec{P}_{\theta}^{\varepsilon} \in \mathbb{C}_{\theta}[X]$, and $\vec{\varphi}_{1}^{\varepsilon}$ satisfies:

$$
\begin{aligned}
& \forall \kappa>0 \quad \frac{\left|\vec{\varphi}_{1}^{\varepsilon}\right|}{\chi^{\theta+2-\kappa}}+\frac{\left|\nabla \vec{\varphi}_{1}^{\varepsilon}\right|}{\chi^{\theta+1-\kappa}} \leq C_{\kappa}\left(C_{0}\right), \\
& \forall p<\infty \quad\left\|\frac{\nabla^{2} \vec{\varphi}_{1}^{\varepsilon}}{\chi^{\theta}}\right\|_{L^{p}(\mathbb{D})} \leq C_{p}\left(C_{0}\right) .
\end{aligned}
$$

Comparing 102 and 111) as in the proof of lemma A.5 (in the appendix) yields:

$$
\begin{aligned}
\vec{P}_{\theta}^{\varepsilon} & =\varepsilon^{\theta}\left[\vec{\Phi}^{1}\left(\frac{z}{\varepsilon}\right)+\vec{Q}^{\varepsilon}\left(\frac{z}{\varepsilon}\right)\right], \\
\vec{\varphi}_{0}^{\varepsilon} & =\vec{p}^{\varepsilon} z^{\theta+1}+\vec{h}_{z}^{\varepsilon}+\vec{\varphi}_{1}^{\varepsilon} .
\end{aligned}
$$

Consequently $\overrightarrow{\varphi_{0}^{\varepsilon}}$ satisfies:

$$
\begin{aligned}
& \frac{\left|\vec{\varphi}_{0}^{\varepsilon}\right|}{\chi^{\theta+1}}+\frac{\left|\nabla \vec{\varphi}_{0}^{\varepsilon}\right|}{\chi^{\theta}} \leq C\left(C_{0}\right) \\
& \forall p<\infty \quad\left\|\frac{\nabla^{2} \vec{\varphi}_{0}^{\varepsilon}}{\chi^{\theta-1}}\right\|_{L^{p}(\mathbb{D})} \leq C_{p}\left(C_{0}\right) .
\end{aligned}
$$

Estimates 113) applied to 102 allow for a pointwise expansion of $\vec{\Phi}_{z z}^{\varepsilon}$ :

$$
\begin{aligned}
\vec{\Phi}_{z}^{\varepsilon} & =\varepsilon^{\theta}\left[\vec{\Phi}_{z}^{1}\left(\frac{z}{\varepsilon}\right)+\vec{Q}^{\varepsilon}\left(\frac{z}{\varepsilon}\right)\right]+\varphi_{0}^{\varepsilon} \\
\vec{\Phi}_{z z}^{\varepsilon} & =\varepsilon^{\theta-1}\left[\vec{\Phi}_{z z}^{1}\left(\frac{z}{\varepsilon}\right)+\vec{Q}_{z}^{\varepsilon}\left(\frac{z}{\varepsilon}\right)\right]+\left(\varphi_{0}^{\varepsilon}\right)_{z} .
\end{aligned}
$$

\section{Step 2: Initial conditions}

The relations (114) yield when evaluated at 0

$$
\begin{aligned}
& \vec{\Phi}_{z}^{\varepsilon}(0)=\varepsilon^{\theta} \vec{\Phi}_{z}^{1}(0)+\varepsilon^{m} \vec{Q}^{\varepsilon}(0)+O\left(\varepsilon^{m+1}\right), \\
& \vec{\Phi}_{z z}^{\varepsilon}(0)=\varepsilon^{\theta-1} \vec{\Phi}_{z z}^{1}(0)+\varepsilon^{\theta-1} \vec{Q}_{z}^{\varepsilon}(0)+O\left(\varepsilon^{m}\right) .
\end{aligned}
$$

Per hypothesis 7, we have:

$$
\begin{aligned}
\vec{\Phi}^{\varepsilon}(0) & =0, \\
\vec{\Phi}_{z}^{\varepsilon}(0) & =\varepsilon^{\theta} \vec{\Phi}_{z}^{1}(0)=\frac{\varepsilon^{\theta}}{2} \vec{e}_{0}, \\
{\left[\Omega^{\varepsilon} e^{-\lambda^{\varepsilon}}\right](0) } & =\frac{1}{\varepsilon^{\theta}} \Omega^{\varepsilon}(0)=-\frac{2}{\varepsilon} .
\end{aligned}
$$

This implies $\vec{n}^{\varepsilon}(0)=-\vec{e}_{3}$, and since $\frac{\Omega^{\varepsilon}}{2}=\left\langle\vec{n}^{\varepsilon}, \vec{\Phi}_{z z}^{\varepsilon}\right\rangle$,

$$
\vec{\Phi}_{z}^{\varepsilon}(0)=\frac{\varepsilon^{\theta}}{2} \vec{e}_{0} \text { and }\left\langle\vec{\Phi}_{z z}^{\varepsilon}(0), \vec{e}_{3}\right\rangle=\varepsilon^{\theta-1} .
$$

Comparing (115) and (116) yields

$$
\vec{Q}^{\varepsilon}(0)=O(\varepsilon) \text { and }\left\langle\vec{Q}_{z}^{\varepsilon}(0), \vec{e}_{3}\right\rangle=O(\varepsilon) .
$$

However as we pointed out in remark A.4.1. $Q^{\varepsilon}$ is loosely defined. We can then evacuate the coefficients of order $\varepsilon$ into $\varphi_{0}^{\varepsilon}$ (which we will do without changing the notations) to obtain:

$$
\vec{Q}^{\varepsilon}(0)=0 \text { and }\left\langle\vec{Q}_{z}^{\varepsilon}(0), \vec{e}_{3}\right\rangle=0 .
$$

To conclude we write $Q^{\varepsilon} \in \mathbb{C}^{3}$ as

$$
\vec{Q}^{\varepsilon}:=A^{\varepsilon} \vec{e}_{0}+B^{\varepsilon} \overline{\vec{e}_{0}}+C^{\varepsilon} \vec{e}_{3},
$$


then 117) yields

$$
\begin{aligned}
& A^{\varepsilon}(0)=B^{\varepsilon}(0)=C^{\varepsilon}(0)=0 \\
& C_{z}^{\varepsilon}(0)=0
\end{aligned}
$$

Evaluating equality 170 (see in the appendix) at $z=0$, one has:

$$
\vec{R}_{z}^{\varepsilon}(0)=\varepsilon^{\theta}\left(H^{\varepsilon}(0)+i V^{\varepsilon}(0)\right) \vec{e}_{0}+i S_{z}^{\varepsilon}(0) \vec{e}_{3}
$$

Estimate (37) then ensures that $\kappa^{\varepsilon}:=\varepsilon^{\theta}\left(H^{\varepsilon}(0)+i V^{\varepsilon}(0)\right)$ is uniformly bounded:

$$
\left|\kappa^{\varepsilon}\right| \leq C\left(C_{0}\right)
$$

\section{Step 3: $\vec{\Phi}^{\varepsilon}$ is conformal}

We will linearize the conformality condition:

$$
\left\langle\vec{\Phi}_{z}^{\varepsilon}, \vec{\Phi}_{z}^{\varepsilon}\right\rangle=0
$$

Injecting 102 in the former yields

$$
Q^{2} B^{\varepsilon}-P^{2} A^{\varepsilon}+P Q C^{\varepsilon}+A^{\varepsilon} B^{\varepsilon}+\frac{\left(C^{\varepsilon}\right)^{2}}{2}=0 .
$$

Applying hypothesis 6 and 118 then yields:

$$
z^{2} \text { divides } B^{\varepsilon}
$$

Conformality also implies

$$
\left\langle\Delta \vec{\Phi}^{\varepsilon}, \vec{\Phi}_{z}^{\varepsilon}\right\rangle=0
$$

Injecting 108 and 114 into the former then yields

$$
\left\langle\widetilde{h}^{\varepsilon}, \varepsilon^{\theta}\left[\vec{\Phi}_{z}^{1}+\vec{Q}^{\varepsilon}\right]\left(\frac{z}{\varepsilon}\right)\right\rangle=\left\langle\widetilde{h}^{\varepsilon}, \vec{\varphi}_{0}^{\varepsilon}\right\rangle+\left\langle\Delta \vec{\Phi}^{\varepsilon}, \vec{\Psi}_{0}^{\varepsilon}\right\rangle=: \vec{\Psi}_{1}^{\varepsilon},
$$

with $\vec{\Psi}_{1}^{\varepsilon}$ satisfying thanks to 109 and 113

$$
\forall \kappa>0 \quad\left|\vec{\Psi}_{1}^{\varepsilon}\right| \leq C_{\kappa} \chi^{2 \theta+1-\kappa}
$$

Considering that $\left\langle\widetilde{h}^{\varepsilon}, \varepsilon^{\theta}\left[\vec{\Phi}_{z}^{1}+\vec{Q}^{\varepsilon}\right]\left(\frac{z}{\varepsilon}\right)\right\rangle$ is a polynomial of degree at most $2 \theta$ in $z$ and $\bar{z}$, we can state:

$$
\left\langle\widetilde{h}^{\varepsilon}, \varepsilon^{\theta}\left[\vec{\Phi}_{z}^{1}+\vec{Q}^{\varepsilon}\right]\left(\frac{z}{\varepsilon}\right)\right\rangle=\sum_{p+q=0}^{2 \theta} \vec{h}_{p q}^{\varepsilon} \varepsilon^{2 \theta-p-q} z^{p} \bar{z}^{q} .
$$

Together 122 and 123 yield:

$$
\forall \kappa>0 \quad \sum_{p+q=0}^{2 \theta} \vec{h}_{p q}^{\varepsilon} \varepsilon^{2 \theta-p-q} z^{p} \bar{z}^{q}=O\left(\chi^{2 \theta+1-\kappa}\right) .
$$

Applying lemma A.7 then yields:

$$
\forall p, q \quad \forall \kappa>0 \quad \vec{h}_{p q}^{\varepsilon}=O\left(\varepsilon^{1-\kappa}\right)
$$

\section{Step 4: Computing $\widetilde{h}^{\varepsilon}$}

We compute

$$
\begin{aligned}
\varepsilon^{\theta} \vec{\Phi}_{\bar{z}}^{1}\left(\frac{z}{\varepsilon}\right)+\varepsilon^{\theta} \overline{\vec{Q}^{\varepsilon}\left(\frac{z}{\varepsilon}\right)} & =\varepsilon^{\theta}\left(\overline{\left(\frac{P^{2}\left(\frac{z}{\varepsilon}\right)}{2}+B^{\varepsilon}\left(\frac{z}{\varepsilon}\right)\right)} \vec{e}_{0}+\varepsilon^{\theta}\left(\overline{\frac{Q^{2}\left(\frac{z}{\varepsilon}\right)}{2}+A^{\varepsilon}\left(\frac{z}{\varepsilon}\right)}\right) \overrightarrow{\vec{e}_{0}}\right. \\
& +\varepsilon^{\theta}\left(\overline{P\left(\frac{z}{\varepsilon}\right) Q\left(\frac{z}{\varepsilon}\right)+C^{\varepsilon}\left(\frac{z}{\varepsilon}\right)}\right) \vec{e}_{3} .
\end{aligned}
$$


Hence

$$
\begin{aligned}
\vec{R}_{z}^{\varepsilon}(0) \times \overline{\varepsilon^{\theta}\left[\vec{\Phi}_{z}^{1}+\vec{Q}^{\varepsilon}\right]\left(\frac{z}{\varepsilon}\right)} & =-2 i \kappa^{\varepsilon} \varepsilon^{\theta} \overline{\left[\frac{Q^{2}}{2}+A^{\varepsilon}\right]\left(\frac{z}{\varepsilon}\right)} \vec{e}_{3}+i \kappa^{\varepsilon} \varepsilon^{\theta} \overline{\left[P Q+C^{\varepsilon}\right]\left(\frac{z}{\varepsilon}\right)} \vec{e}_{0} \\
& +S_{z}^{\varepsilon}(0) \varepsilon^{\theta} \overline{\left[-\frac{P^{2}}{2}+B^{\varepsilon}\right]\left(\frac{z}{\varepsilon}\right)} \vec{e}_{0}-S_{z}^{\varepsilon}(0) \varepsilon^{\theta} \overline{\left[\frac{Q^{2}}{2}+A^{\varepsilon}\right]\left(\frac{z}{\varepsilon}\right)}
\end{aligned}
$$

and

$$
\begin{aligned}
S_{z}^{\varepsilon}(0) \overline{\varepsilon^{\theta}\left[\vec{\Phi}_{z}^{1}+\vec{Q}^{\varepsilon}\right]\left(\frac{z}{\varepsilon}\right)} & =S_{z}^{\varepsilon}(0) \varepsilon^{\theta} \overline{\left[-\frac{P^{2}}{2}+B^{\varepsilon}\right]\left(\frac{z}{\varepsilon}\right)} \vec{e}_{0}+S_{z}^{\varepsilon}(0) \varepsilon^{\theta} \overline{\left[\frac{Q^{2}}{2}+A^{\varepsilon}\right]\left(\frac{z}{\varepsilon}\right)} \overline{\vec{e}_{0}} \\
& +S_{z}^{\varepsilon}(0) \varepsilon^{\theta} \overline{\left[P Q+C^{\varepsilon}\right]\left(\frac{z}{\varepsilon}\right)} \vec{e}_{3} .
\end{aligned}
$$

Then

$$
\begin{aligned}
\widetilde{h}^{\varepsilon} & =2 \Im\left(\left(S_{z}^{\varepsilon}(0) \varepsilon^{\theta} \overline{\left[-P^{2}+2 B^{\varepsilon}\right]\left(\frac{z}{\varepsilon}\right)}+i \kappa^{\varepsilon} \varepsilon^{\theta} \overline{\left[P Q+C^{\varepsilon}\right]\left(\frac{z}{\varepsilon}\right)}\right) \vec{e}_{0}\right. \\
& \left.+\left(S_{z}^{\varepsilon}(0) \varepsilon^{\theta} \overline{\left[P Q+C^{\varepsilon}\right]\left(\frac{z}{\varepsilon}\right)}-i \kappa^{\varepsilon} \varepsilon^{\theta} \overline{\left[Q^{2}+2 A^{\varepsilon}\right]\left(\frac{z}{\varepsilon}\right)}\right) \vec{e}_{3}\right) .
\end{aligned}
$$

From this we deduce

$$
\begin{aligned}
\left\langle\widetilde{h}^{\varepsilon}, \varepsilon^{\theta}\left[\vec{\Phi}_{z}^{1}+\vec{Q}^{\varepsilon}\right]\left(\frac{z}{\varepsilon}\right)\right\rangle & =\left(S_{z}^{\varepsilon}(0) \varepsilon^{\theta} \overline{\left[-P^{2}+2 B^{\varepsilon}\right]\left(\frac{z}{\varepsilon}\right)}+i \kappa^{\varepsilon} \varepsilon^{\theta} \theta \overline{\left[P Q+C^{\varepsilon}\right]\left(\frac{z}{\varepsilon}\right)}\right) \varepsilon^{\theta}\left[-P^{2}+2 B^{\varepsilon}\right]\left(\frac{z}{\varepsilon}\right) \\
& +\left(S_{z}^{\varepsilon}(0) \varepsilon^{\theta} \overline{\left[P Q+C^{\varepsilon}\right]\left(\frac{z}{\varepsilon}\right)}-i \kappa^{\varepsilon} \varepsilon^{\theta} \overline{\left[Q^{2}+2 A^{\varepsilon}\right]\left(\frac{z}{\varepsilon}\right)}\right) \varepsilon^{\theta}\left[P Q+C^{\varepsilon}\right]\left(\frac{z}{\varepsilon}\right) \\
& =S_{z}^{\varepsilon}(0) \varepsilon^{2 \theta}\left[|P|^{2}\left(|P|^{2}+|Q|^{2}\right)+2 \Re\left(P Q \overline{C^{\varepsilon}}-2 P^{2} \overline{B^{\varepsilon}}\right)+4\left|B^{\varepsilon}\right|^{2}+\left|C^{\varepsilon}\right|^{2}\right]\left(\frac{z}{\varepsilon}\right) \\
& +i \kappa^{\varepsilon} \varepsilon^{2 \theta}\left[-P \bar{Q}\left(|P|^{2}+|Q|^{2}\right)-P^{2} \overline{C^{\varepsilon}}+2 B^{\varepsilon} \overline{P Q}-2 P Q \overline{A^{\varepsilon}}\right. \\
& \left.-C^{\varepsilon} \overline{Q^{2}}+2 B^{\varepsilon} \overline{C^{\varepsilon}}-2 C^{\varepsilon} \overline{A^{\varepsilon}}\right]\left(\frac{z}{\varepsilon}\right) .
\end{aligned}
$$

Studying 125 with (118), 121), 122, 123 and hypothesis 6 in mind, we can write

$$
\left\langle\widetilde{h}^{\varepsilon}, \varepsilon^{\theta}\left[\vec{\Phi}_{z}^{1}+\vec{Q}^{\varepsilon}\right]\left(\frac{z}{\varepsilon}\right)\right\rangle=-i \kappa^{\varepsilon} \varepsilon^{2 \theta-1} z+O\left(r^{2}\right)
$$

which implies $h_{1,0}^{\varepsilon}=-i \kappa^{\varepsilon}$, and in turn thanks to 124$)$ :

$$
\forall s>0 \quad \kappa^{\varepsilon}=O\left(\varepsilon^{1-s}\right)
$$

Then, 122, 123 and 125 give us:

$$
\begin{aligned}
\left\langle\widetilde{h}^{\varepsilon}, \varepsilon^{\theta}\left[\vec{\Phi}_{z}^{1}+\vec{Q}^{\varepsilon}\right]\left(\frac{z}{\varepsilon}\right)\right\rangle & =S_{z}^{\varepsilon}(0) \varepsilon^{2 \theta}\left[|P|^{2}\left(|P|^{2}+|Q|^{2}\right)+2 \Re\left(P Q \overline{C^{\varepsilon}}-2 P^{2} \overline{B^{\varepsilon}}\right)+4\left|B^{\varepsilon}\right|^{2}+\left|C^{\varepsilon}\right|^{2}\right]\left(\frac{z}{\varepsilon}\right) \\
& +O\left(\chi^{2 \theta+1-\kappa}\right) .
\end{aligned}
$$

A similar process on the remaining polynomial allows us to state

$$
\forall \kappa>0 \quad S_{z}^{\varepsilon}(0)=O\left(\varepsilon^{1-\kappa}\right) .
$$

\section{Step 5: Conclusion}

From (106), (127) and $(128)$ we deduce:

$$
\begin{aligned}
& \forall \kappa>0 \quad\left|\vec{R}_{z}^{\varepsilon}\right|+\left|S_{z}^{\varepsilon}\right| \leq C_{\kappa} \chi^{1-\kappa}, \\
& \forall p<\infty \quad\left\|\nabla \vec{R}_{z}^{\varepsilon}\right\|_{L^{p}(\mathbb{D})}+\left\|\nabla S_{z}^{\varepsilon}\right\|_{L^{p}(\mathbb{D})} \leq C_{p} .
\end{aligned}
$$

Inequality (119) from [19] then yields:

$$
\forall \kappa>0 \quad\left|H^{\varepsilon} e^{\lambda^{\varepsilon}}\right| \leq C_{\kappa} \chi^{1-\kappa}
$$


Letting $\varepsilon$ tend to 0 in 131 gives, for any $r>0$, thanks to hypothesis 2 , the following:

$$
\forall \kappa>0 \quad\left|H^{0} e^{\lambda^{0}}\right| \leq C_{\kappa} r^{1-\kappa}
$$

However since $\vec{\Phi}^{0}$ is assumed to have a branch point of order $\theta+1$ at 0 , by definition, $e^{\lambda^{0}} \sim C r^{\theta}$, which means

$$
\forall \kappa>0 \quad\left|H^{0}\right| \leq C_{\kappa} r^{1-\theta-\kappa} .
$$

By definition of $\alpha($ see $(33)), H^{0} \simeq r^{-\alpha}$. Since $\alpha \in \mathbb{Z}, 132$ ensures:

$$
\alpha \leq \theta-1
$$

This concludes the proof of the desired result.

In the continuity of the previous proof we can improve on a convergence result obtained in [19]:

Theorem 5.2. Let $\vec{\Phi}^{k}: \Sigma \rightarrow \mathbb{R}^{3}$ be a sequence of Willmore immersions satisfying the hypotheses of theorem 1.1. Assume further that at each concentration point a simple minimal bubble is blown. Then $\vec{\Phi}^{k} \rightarrow \vec{\Phi}^{0} C^{3, \eta}$ for all $\eta<1$.

Proof. As before we can reason locally, under hypotheses 1,7, and will continue from 133. Injecting 130 into 108 ensures:

$$
\begin{array}{ll}
\forall \kappa>0 & \left|\Delta \vec{\Phi}^{\varepsilon}\right| \leq C_{\kappa} \chi^{\theta+1-\kappa} \\
\forall p<\infty & \left\|\frac{\nabla\left(\Delta \vec{\Phi}^{\varepsilon}\right)}{\chi^{\theta}}\right\|_{L^{p}(\mathbb{D})} \leq C_{p} .
\end{array}
$$

We can then compute

$$
\begin{aligned}
& H^{\varepsilon} \vec{\Phi}_{z}^{\varepsilon}=\frac{\vec{\Phi}_{z \bar{z}}^{\varepsilon} \times \vec{\Phi}_{z}^{\varepsilon}}{i\left|\vec{\Phi}_{z}^{\varepsilon}\right|^{2}} \\
& \nabla\left(H^{\varepsilon} \vec{\Phi}_{z}^{\varepsilon}\right)=\frac{\nabla\left(\vec{\Phi}_{z \bar{z}}^{\varepsilon}\right) \times \vec{\Phi}_{z}^{\varepsilon}}{i\left|\vec{\Phi}_{z}^{\varepsilon}\right|^{2}}+\frac{\vec{\Phi}_{z \bar{z}}^{\varepsilon} \times \nabla\left(\vec{\Phi}_{z}^{\varepsilon}\right)}{i\left|\vec{\Phi}_{z}^{\varepsilon}\right|^{2}}-\left(\left\langle\nabla \vec{\Phi}_{z}^{\varepsilon}, \vec{\Phi}_{\bar{z}}\right\rangle+\left\langle\vec{\Phi}_{z}, \nabla \vec{\Phi}_{\bar{z}}\right\rangle\right) \frac{\vec{\Phi}_{z \bar{z}}^{\varepsilon} \times \vec{\Phi}_{z}^{\varepsilon}}{i\left|\vec{\Phi}_{z}^{\varepsilon}\right|^{4}} .
\end{aligned}
$$

Combining 100, 114) and 134) yields:

$$
\begin{aligned}
& \forall \kappa>0 \quad\left\|\frac{H^{\varepsilon} \vec{\Phi}_{z}^{\varepsilon}}{\chi^{1-\kappa}}\right\|_{L^{\infty}(\mathbb{D})} \leq C_{\kappa}, \\
& \forall p<\infty \quad\left\|\nabla\left(H^{\varepsilon} \vec{\Phi}_{z}^{\varepsilon}\right)\right\|_{L^{p}(\mathbb{D})} \leq C_{p} .
\end{aligned}
$$

Consequently, injecting (136) into 104 and applying Calderon-Zygmund yields

$$
\forall p<\infty \quad\left\|\nabla S^{\varepsilon}\right\|_{W^{2, p}(\mathbb{D})}+\left\|\nabla \vec{R}^{\varepsilon}\right\|_{W^{2, p}(\mathbb{D})}+\left\|\nabla \vec{\Phi}^{\varepsilon}\right\|_{W^{3, p}(\mathbb{D})} \leq C\left(C_{0}\right)
$$

Which proves theorem 5.2 thanks to classical embeddings.

Remark 5.2.1. We can extend our expansions to the next order. Indeed injecting (136) and (130 into 104 yields

$$
\begin{aligned}
& \forall \kappa>0 \quad\left\|\frac{\Delta S^{\varepsilon}}{\chi^{2-\kappa}}\right\|_{L^{\infty}(\mathbb{D})}+\left\|\frac{\Delta \vec{R}^{\varepsilon}}{\chi^{2-\kappa}}\right\|_{L^{\infty}(\mathbb{D})} \leq C_{\kappa}, \\
& \forall p<\infty \quad\left\|\frac{\Delta \nabla S^{\varepsilon}}{\chi}\right\|_{L^{p}(\mathbb{D})}+\left\|\frac{\Delta \nabla \vec{R}^{\varepsilon}}{\chi}\right\|_{L^{p}(\mathbb{D})} \leq C_{p} .
\end{aligned}
$$


Applying corollary A.8 (see in the appendix) then yields

$$
\begin{aligned}
& S_{z}^{\varepsilon}=S_{z}^{\varepsilon}(0)+s_{1}^{\varepsilon} z+s_{2}^{\varepsilon} z^{2}+\sigma_{1}^{\varepsilon}, \\
& \vec{R}_{z}^{\varepsilon}=\vec{R}_{z}^{\varepsilon}(0)+\vec{r}_{1}^{\varepsilon} z+\vec{r}_{2}^{\vec{\varepsilon}} z^{2}+\vec{\rho}_{1}^{\varepsilon},
\end{aligned}
$$

where the $s_{j}^{\varepsilon}$ and the $\vec{r}_{j}^{\varepsilon}$ are uniformly bounded constants and $\sigma_{1}^{\varepsilon}, \rho_{1}^{\varepsilon}$ satisfy:

$$
\begin{aligned}
& \forall \kappa>0 \quad\left|\frac{\sigma_{1}^{\varepsilon}}{\chi^{3-\kappa}}\right|+\left|\frac{\nabla \sigma_{1}^{\varepsilon}}{\chi^{2-\kappa}}\right|+\left|\frac{\overrightarrow{\rho_{1}^{\varepsilon}}}{\chi^{3-\kappa}}\right|+\left|\frac{\nabla \overrightarrow{\rho_{1}^{\varepsilon}}}{\chi^{2-\kappa}}\right| \leq C_{\kappa}, \\
& \forall p<\infty \quad\left\|\frac{\nabla^{2} \sigma_{1}^{\varepsilon}}{\chi}\right\|_{L^{p}(\mathbb{D})}+\left\|\frac{\nabla^{2} \overrightarrow{\rho_{1}^{\varepsilon}}}{\chi}\right\|_{L^{p}(\mathbb{D})} \leq C_{p} .
\end{aligned}
$$

Setting $\sigma_{0}^{\varepsilon}=s_{1}^{\varepsilon} z+s_{2}^{\varepsilon} z^{2}+\sigma_{1}^{\varepsilon}$ and $\overrightarrow{\rho_{0}^{\varepsilon}}=\overrightarrow{r_{1}^{\varepsilon}} z+\overrightarrow{r_{2}^{\varepsilon}} z^{2}+\vec{\rho}_{1}^{\varepsilon}$ yields

$$
\begin{aligned}
& \left|\frac{\sigma_{0}^{\varepsilon}}{\chi}\right|+\left|\nabla \sigma_{0}^{\varepsilon}\right|+\left|\frac{\vec{\rho}_{0}^{\varepsilon}}{\chi}\right|+\left|\nabla \bar{\rho}_{0}^{\varepsilon}\right| \leq C, \\
& \forall p<\infty \quad\left\|\nabla^{2} \sigma_{0}^{\varepsilon}\right\|_{L^{p}(\mathbb{D})}+\left\|\nabla^{2} \bar{\rho}_{0}^{\varepsilon}\right\|_{L^{p}(\mathbb{D})} \leq C_{p} .
\end{aligned}
$$

We can then do all the reasonings from 108 to 136 for better controls:

$$
\left|\frac{H^{\varepsilon} \vec{\Phi}_{z}^{\varepsilon}}{\chi}\right|+\left|\frac{S_{z}^{\varepsilon}}{\chi}\right|+\left|\frac{\vec{R}_{z}^{\varepsilon}}{\chi}\right| \leq C .
$$

Injecting this added regularity into the third equation of 104 ensures:

$$
\begin{aligned}
& \left|\frac{\Delta \vec{\Phi}^{\varepsilon}}{\chi^{3}}\right|+\left|\frac{\Delta \nabla \vec{\Phi}}{\chi^{2}}\right| \leq C \\
& \forall p<\infty \quad\left\|\frac{\Delta \nabla^{2} \vec{\Phi}}{\chi}\right\|_{L^{p}(\mathbb{D})} \leq C_{p} .
\end{aligned}
$$

With another application of corollary A.8 (see in the appendix) we can expand $\vec{\Phi}_{z}^{\varepsilon}$ in the following manner:

$$
\vec{\Phi}_{z}^{\varepsilon}=\varepsilon^{\theta}\left[\vec{\Phi}_{z}^{1}\left(\frac{z}{\varepsilon}\right)+\vec{Q}^{\varepsilon}\left(\frac{z}{\varepsilon}\right)\right]+\vec{\varphi}_{0}^{\varepsilon},
$$

with

$$
\begin{aligned}
& \frac{\left|\varphi_{0}^{\varepsilon}\right|}{\chi^{\theta+1}}+\frac{\left|\nabla \varphi_{0}^{\varepsilon}\right|}{\chi^{\theta}}+\frac{\left|\nabla^{2} \varphi_{0}^{\varepsilon}\right|}{\chi^{\theta-1}} \leq C\left(C_{0}\right) \\
& \forall p<\infty \quad\left\|\frac{\nabla^{3} \varphi_{0}^{\varepsilon}}{\chi^{\theta-2}}\right\|_{L^{p}(\mathbb{D})} \leq C_{p}\left(C_{0}\right) .
\end{aligned}
$$

\subsection{Proof of corollary $\mathbf{1 . 6}$}

Proof. We only need to show that the inverse of a Chen-Gackstatter torus has a branch point of second residue $\alpha=2$. Let $\vec{\Psi}:\left(\mathbb{C} \backslash \mathbb{Z}^{2}\right) / \mathbb{Z}^{2} \rightarrow \mathbb{R}^{3}$ be a parametrization of the Chen-Gackstatter torus, $p \in \mathbb{R}^{3}$ such that $d(p, \vec{\Psi})>1$, and $\vec{\Phi}=\iota \circ(\vec{\Psi}-p)$, the studied inverse. Let $b$ denote its branch point.

It is interesting to notice that the second residue of $\vec{\Phi}$ can be read on its conformal Gauss map $Y$ defined as:

$$
Y_{\vec{\Phi}}=H_{\vec{\Phi}}\left(\begin{array}{c}
\vec{\Phi} \\
\frac{|\vec{\Phi}|^{2}-1}{|\vec{\Phi}|^{2}+1} \\
2
\end{array}\right)+\left(\begin{array}{c}
\vec{n}_{\vec{\Phi}} \\
\left\langle\vec{n}_{\vec{\Phi}}, \vec{\Phi}\right\rangle \\
\left.\vec{n}_{\vec{\Phi}}, \vec{\Phi}\right\rangle
\end{array}\right) .
$$

Indeed $\vec{\Phi}$ and $\vec{n}_{\vec{\Phi}}$ are bounded around the branch point, and thus necessarily

$$
Y_{\vec{\Phi}} \sim_{b} C_{\vec{\Phi}} z^{-\alpha}
$$


Further, since $\vec{\Phi}$ and $\vec{\Psi}$ differ by a conformal transform, it has been shown in [18, that there exists a fixed matrix $M \in S O(4,1)$ such that $Y_{\vec{\Psi}}=M Y_{\vec{\Phi}}$. This yields that necessarily $Y_{\vec{\Psi}} \sim_{b} C_{\vec{\Psi}} z^{-\alpha}$. Hence, considering that $\vec{\Psi}$ is minimal, we deduce that

$$
Y_{\vec{\Psi}}=\left(\begin{array}{c}
\vec{n}_{\vec{\Psi}} \\
\left\langle\vec{n}_{\vec{\Psi}}, \vec{\Psi}\right\rangle \\
\left\langle\vec{n}_{\vec{\Psi}}, \vec{\Psi}\right\rangle
\end{array}\right) .
$$

Since $\vec{n}_{\Psi}$ is bounded,

$$
\left\langle\vec{n}_{\vec{\Psi}}, \vec{\Psi}\right\rangle \sim_{b} C z^{-\alpha}
$$

We will now use the Enneper-Weierstrass parametrization of $\vec{\Psi}$ and $(145)$ to compute the second residue of $\vec{\Psi}$ at its branch point. Chen-Gackstatter is a minimal surface of genus 1 and of Enneper-Weierstrass data centered on the branch point: $(f, g)=\left(2 \varphi(z), A \frac{\varphi_{z}}{\varphi}(z)\right)$ (see [8]) where $\varphi$ is the Weierstrass elliptic function, of elliptic invariants

$$
\begin{aligned}
& g_{2}=60 \sum_{m, n=-\infty}^{\infty} \frac{1}{(m+n i)^{4}}>0, \\
& g_{3}=0,
\end{aligned}
$$

and

$$
A=\sqrt{\frac{3 \pi}{2 g_{2}}} \in \mathbb{R}_{+} .
$$

Then, $\varphi$ has the following expansion around 0 (see [1]):

$$
\begin{gathered}
\varphi(z)=\frac{1}{z^{2}}+O\left(z^{2}\right) \\
\varphi_{z}(z)=\frac{-2}{z^{3}}+O(z) .
\end{gathered}
$$

Hence we can state that

$$
\begin{aligned}
\vec{\Psi} & =2 \Re\left(\int \frac{1}{z^{2}} \vec{e}_{0}-\frac{4 A^{2}}{z^{4}} \overrightarrow{\vec{e}_{0}}-\frac{4 A}{z^{3}} \vec{e}_{3}+O(1) d z\right) \\
& =2 \Re\left(\frac{4 A^{2}}{3 z^{3}} \overrightarrow{\vec{e}_{0}}-\frac{1}{z} \vec{e}_{0}+\frac{2 A}{z^{2}} \vec{e}_{3}+O(z)\right) \\
& =\left(\frac{4 A^{2}}{3 \bar{z}^{3}}-\frac{1}{z}\right) \vec{e}_{0}+\left(\frac{4 A^{2}}{3 z^{3}}-\frac{1}{\bar{z}}\right) \overrightarrow{\vec{e}_{0}}+2 A\left(\frac{1}{z^{2}}+\frac{1}{\bar{z}^{2}}\right) \vec{e}_{3}+O(r) .
\end{aligned}
$$

Similarly:

$$
\begin{aligned}
\vec{\Psi}_{z} \times \vec{\Psi}_{\bar{z}} & =\left(\frac{1}{z^{2}} \vec{e}_{0}-\frac{4 A^{2}}{z^{4}} \overrightarrow{\vec{e}_{0}}-\frac{4 A}{z^{3}} \vec{e}_{3}+O(1)\right) \times\left(\frac{1}{\bar{z}^{2}} \overrightarrow{\vec{e}_{0}}-\frac{4 A^{2}}{\bar{z}^{4}} \overrightarrow{e_{0}}-\frac{4 A}{\bar{z}^{3}} \overrightarrow{e_{3}}+O(1)\right) \\
& =-\frac{4 A i}{z^{2} \bar{z}^{3}} \vec{e}_{0}+\frac{32 i A^{4}}{r^{8}} \vec{e}_{3}-\frac{16 A^{3} i}{z^{4} \bar{z}^{3}} \overrightarrow{\vec{e}_{0}}-\frac{4 A i}{z^{3} \bar{z}^{2}} \overline{\vec{e}_{0}}-\frac{16 A^{3} i}{z^{3} \bar{z}^{4}} \vec{e}_{0}+O\left(\frac{1}{r^{4}}\right) \\
& =\frac{32 i A^{4}}{r^{8}}\left(-\left(\frac{z}{2 A}+\frac{z^{2} \bar{z}}{8 A^{3}}\right) \vec{e}_{0}-\left(\frac{\bar{z}}{2 A}+\frac{\bar{z}^{2} z}{8 A^{3}}\right) \overrightarrow{\vec{e}_{0}}+\vec{e}_{3}+O\left(r^{4}\right)\right)
\end{aligned}
$$

and

$$
\begin{aligned}
\left|\vec{\Psi}_{z}\right|^{2} & =\frac{32 A^{4}}{r^{8}}+\frac{16 A^{2}}{r^{6}}+O\left(r^{-4}\right) \\
& =\frac{32 A^{4}}{r^{8}}\left(1+\frac{r^{2}}{2 A^{2}}+O\left(r^{4}\right)\right),
\end{aligned}
$$

which yields

$$
\vec{n}_{\vec{\Psi}}=\left(1-\frac{r^{2}}{2 A^{2}}\right) \vec{e}_{3}-\left(\frac{z}{2 A}-\frac{z^{2} \bar{z}}{8 A^{3}}\right) \vec{e}_{0}-\left(\frac{\bar{z}}{2 A}-\frac{z \bar{z}^{2}}{8 A^{3}}\right) \overline{\vec{e}_{0}}+O\left(r^{4}\right) .
$$

Combining 146) and 147) ensures:

$$
\left\langle\vec{n}_{\vec{\Psi}}, \vec{\Psi}\right\rangle=2 A\left(\frac{1}{z^{2}}+\frac{1}{\bar{z}^{2}}\right)-\frac{4 A}{3 z^{2}}-\frac{4 A}{3 \bar{z}^{2}}+O\left(\frac{1}{r}\right)=\frac{2 A}{3 z^{2}}+\frac{2 A}{3 \bar{z}^{2}}+O\left(\frac{1}{r}\right) .
$$

Considering 148 in light of 145 yields $\alpha=2$. Applying theorem 1.5 concludes the proof. 


\subsection{An exploration of the consequences of theorem 1.5}

We consider $\vec{\Phi}: \mathbb{D} \rightarrow \mathbb{R}^{3}$ a true Willmore conformal branched immersion with a single branch point at 0 , of multiplicity $\theta+1$, and of second residue $\alpha \leq \theta-1$. Then applying theorem 1.4 we can expand $\vec{\Phi}$ around 0 in the following way:

$$
\begin{aligned}
\vec{\Phi}(z) & =2 \Re\left(\frac{1}{2(\theta+1)} \overline{\vec{e}_{0}} z^{\theta+1}+\sum_{j=1}^{\theta+1-\alpha} \frac{\vec{A}_{j}}{\theta+1+j} z^{\theta+1+j}\right. \\
& \left.+\left(\frac{C z^{\theta+1-\alpha} \bar{z}^{\theta+1}+\bar{C} z^{\theta+1} \bar{z}^{\theta+1-\alpha}}{(\theta+1-\alpha)(\theta+1)}\right) \vec{e}_{3}\right)+\vec{\xi}
\end{aligned}
$$

where $\vec{\xi}$ satisfies

$$
\nabla^{j} \vec{\xi}=O\left(|z|^{2(\theta+1}-\alpha-j+1-\nu\right) \text { for all } \kappa>0 \text { and } j \leq \theta+2-\alpha .
$$

Moreover, if we do the conformal change of variables $Z^{\theta+1}=z^{\theta+1}+A z^{\theta+2}, 149$ becomes

$$
\begin{aligned}
\vec{\Phi}(Z) & =2 \Re\left(\frac{1}{2(\theta+1)} \overrightarrow{\vec{e}_{0}} Z^{\theta+1}+\left(\frac{\overrightarrow{A_{1}}}{\theta+2}+A \overrightarrow{\vec{e}_{0}}\right) Z^{\theta+2}\right. \\
& \left.+\left(\frac{C Z^{\theta+1-\alpha} \bar{Z}^{\theta+1}+\bar{C} Z^{\theta+1} \bar{Z}^{\theta+1-\alpha}}{(\theta+1-\alpha)(\theta+1)}\right) \vec{e}_{3}\right)+O\left(|Z|^{\theta+3}\right) .
\end{aligned}
$$

Thus up to doing a conformal change of charts we can assume that $\overrightarrow{A_{1}}$ has no component along $\overrightarrow{\vec{e}_{0}}$, meaning:

$$
\overrightarrow{A_{1}}=\frac{V}{2} \vec{e}_{0}+U \vec{e}_{3}
$$

Then

$$
\vec{\Phi}_{z}=\frac{1}{2} \overrightarrow{\vec{e}_{0}} z^{\theta}+\sum_{j=1}^{\theta+1-\alpha} \vec{A}_{j} z^{\theta+j}+\left(\frac{C}{\theta+1} z^{\theta-\alpha} \bar{z}^{\theta+1}+\frac{\bar{C}}{\theta+1-\alpha} z^{\theta} \bar{z}^{\theta+1-\alpha}\right) \vec{e}_{3}+\vec{\xi}_{z},
$$

Using $\vec{\Phi}$ conformal, we can expand $\left\langle\vec{\Phi}_{z}, \vec{\Phi}_{z}\right\rangle$ to the order $z^{2 \theta+1}$ and conclude that

$$
V=\left\langle\overrightarrow{A_{1}}, \overrightarrow{\vec{e}_{0}}\right\rangle=0
$$

Then, wishing to expand the Gauss map $\vec{n}$ we compute:

$$
\begin{gathered}
\left|\vec{\Phi}_{z}\right|^{2}=\frac{r^{2 \theta}}{2}+O\left(r^{2 \theta+2}\right), \\
\vec{\Phi}_{z} \times \vec{\Phi}_{\bar{z}}=\left(\frac{1}{2} \overrightarrow{\vec{e}_{0}}+U z^{\theta+1} \vec{e}_{3}+O\left(r^{\theta+2}\right)\right) \times\left(\frac{1}{2} \vec{e}_{0}+\bar{U} \bar{z}^{\theta+1} \vec{e}_{3}+O\left(r^{\theta+2}\right)\right) \\
=\frac{i r^{2 \theta}}{2} \vec{e}_{3}-\frac{i U z^{\theta+1} \bar{z}^{\theta}}{2} \vec{e}_{0}-\frac{i \bar{U} z^{\theta} \bar{z}^{\theta+1}}{2} \overrightarrow{\vec{e}_{0}}+O\left(r^{2 \theta+2}\right) .
\end{gathered}
$$

Hence we can write

$$
\vec{n}=\vec{e}_{3}-U z \vec{e}_{0}-\bar{U} \bar{z} \overline{\vec{e}_{0}}+O\left(r^{2}\right) .
$$

One can differentiate 151), and obtain

$$
\begin{aligned}
\vec{\Phi}_{z z} & =\frac{\theta}{2} \overrightarrow{\vec{e}_{0}} z^{\theta-1}+\sum_{j=1}^{\theta+1-\alpha}(\theta+j) \vec{A}_{j} z^{\theta-1+j} \\
& +\left(\frac{(\theta-\alpha) C}{\theta+1} z^{\theta-1-\alpha} \bar{z}^{\theta+1}+\frac{\theta \bar{C}}{\theta+1-\alpha} z^{\theta-1} \bar{z}^{\theta+1-\alpha}\right) \vec{e}_{3}+\vec{\xi}_{z z} .
\end{aligned}
$$

Taking the scalar product with 152 we find:

$$
\frac{\Omega}{2}=(\theta+1) U z^{\theta}-\theta U z^{\theta}+O\left(r^{\theta+1}\right)=U z^{\theta}+O\left(r^{\theta+1}\right) .
$$


In theorem 4.11 of [20, A. Michelat and T. Rivière have shown that

$$
\left\langle\overrightarrow{A_{1}}, C \overrightarrow{e_{3}}\right\rangle=U C=0
$$

This precise equality is found at (4.53), (4.54) and (4.69) (depending on whether $\theta \geq 4, \theta=3$ or $\theta=2$ ) of the aforementioned article and stems from the conformal relation $\left\langle\vec{\Phi}_{z}, \vec{\Phi}_{z}\right\rangle=0$, expanded to the order $3 \theta+2-\alpha$ (which requires furthering expansion (151) in the way detailed in 20]) in order to consider the first terms in $\bar{z}$. Equality 153 implies notably that either $C=0$, and thus that $\alpha \leq \theta-2$, of $U=0$, and thus that $|\Omega| e^{-\lambda}=O(r)$, meaning that 0 is an umbilic point. Besides, theorem 4.11 of [20] states that Bryant's quartic (see in the introduction, or in [18]) is then holomorphic across the branched point.

Theorem 1.5 has the following corollary:

Corollary 5.3. Let $\vec{\Phi}_{k}$ be a sequence of Willmore immersions of a closed surface $\Sigma$ satisfying the hypotheses of theorem 1.1. Then at each concentration point $p \in \Sigma$ of multiplicity $\theta_{p}+1$ on which a simple minimal bubble is blown, either the second residue of $\vec{\Phi}_{\infty}$ at $p$ satisfies

$$
\alpha_{p} \leq \theta_{p}-2
$$

or $p$ is an umbilic point for $\vec{\Phi}_{\infty}(\Sigma)$.

In both cases Bryant's quartic $\mathcal{Q}$ is holomorphic across those branch points.

\section{A Appendix}

\section{A.1 Weighted Calderon-Zygmund}

Theorems A.1 and A.2 are taken from Y. Bernard and T. Rivière's [3] (Proposition C.2 and C.3). They were not originally stated with the case $p=\infty$, but the proof is the same.

Theorem A.1. Let $u \in C^{2}(\mathbb{D} \backslash\{0\})$ solve

$$
\Delta u(z)=\mu(z) f(z) \text { in } \mathbb{D},
$$

with $f \in L^{p}(\mathbb{D})$ for $2<p \leq \infty$ and the weight $\mu$ satisfying for some $a \in \mathbb{N}$

$$
|\mu(z)|=O\left(|z|^{a}\right) .
$$

Then

$$
u_{z}(z)=P(z)+|z|^{a} T(z)
$$

with $P \in \mathbb{C}_{a}[X]$ and $T=O\left(|z|^{1-\frac{2}{p}-\kappa}\right)$ for all $\kappa>0$. More precisely one has

$$
\left\|\frac{T}{|z|^{1-\frac{2}{p}-\kappa}}\right\|_{L^{\infty}(\mathbb{D})} \leq C_{\kappa}\left(\left\|\frac{\mu}{|z|^{a}}\right\|_{L^{\infty}(\mathbb{D})}\|f\|_{L^{p}(\mathbb{D})}+\|u\|_{C^{1}(\partial \mathbb{D})}\right) .
$$

Additionally if $\mu \in C^{1}(\mathbb{D} \backslash\{0\}), a \neq 0$ and

$$
\nabla \mu(z)=O\left(|z|^{a-1}\right)
$$

Then:

$$
u_{z z}(z)=P_{z}+|z|^{a} Q
$$

with $Q \in L^{p^{\prime}}(\mathbb{D})$ for all $p^{\prime}<p$ and

$$
\|Q\|_{L^{p^{\prime}}(\mathbb{D})} \leq C_{p^{\prime}}\left(\left(\left\|\frac{\mu}{|z|^{a}}\right\|_{L^{\infty}(\mathbb{D})}+\left\|\frac{\nabla \mu}{|z|^{a-1}}\right\|_{L^{\infty}(\mathbb{D})}\right)\|f\|_{L^{p}(\mathbb{D})}+\|u\|_{C^{1}(\partial \mathbb{D})}\right) .
$$

In fact $Q=\frac{\left(|z|^{a} T(z)\right)_{z}}{|z|^{a}}$.

Remark A.1.1. Theorem A.1 works with $a=0$, it is the classic Calderon-Zygmund theorem. 
Theorem A.2. Let $u \in C^{2}(\mathbb{D} \backslash\{0\})$ solve

$$
\Delta u(z)=\mu(z) f(z) \text { in } \mathbb{D},
$$

with $f \in L^{p}(\mathbb{D})$ for $2<p \leq \infty$ and the weight $\mu$ satisfying for some $a \in \mathbb{R}_{+}$

$$
|\mu(z)|=O\left(|z|^{a}\right) .
$$

Then

$$
u_{z}(z)=P(z)+|z|^{a} T(z)
$$

with $P \in \mathbb{C}_{\lceil a\rceil}[X]$ and $T=O\left(|z|^{1-\frac{2}{p}-\kappa}\right)$ for all $\kappa>0$. Here $\lceil a\rceil$ is the upper integral part of $a$. More precisely one has

$$
\left\|\frac{T}{|z|^{1-\frac{2}{p}-\kappa}}\right\|_{L^{\infty}(\mathbb{D})} \leq C_{\kappa}\left(\left\|\frac{\mu}{|z|^{a}}\right\|_{L^{\infty}(\mathbb{D})}\|f\|_{L^{p}(\mathbb{D})}+\|u\|_{C^{1}(\partial \mathbb{D})}\right) .
$$

Additionally if $\mu \in C^{1}(\mathbb{D} \backslash\{0\}), a \neq 0$ and

$$
\nabla \mu(z)=O\left(|z|^{a-1}\right)
$$

Then:

$$
u_{z z}(z)=P_{z}+|z|^{a} Q
$$

with $Q \in L^{p^{\prime}}(\mathbb{D})$ for all $p^{\prime}<p$ and

$$
\|Q\|_{L^{p^{\prime}(\mathbb{D})}} \leq C_{p^{\prime}}\left(\left(\left\|\frac{\mu}{|z|^{a}}\right\|_{L^{\infty}(\mathbb{D})}+\left\|\frac{\nabla \mu}{|z|^{a-1}}\right\|_{L^{\infty}(\mathbb{D})}\right)\|f\|_{L^{p}(\mathbb{D})}+\|u\|_{C^{1}(\partial \mathbb{D})}\right) .
$$

In fact $Q=\frac{\left(|z|^{a} T(z)\right)_{z}}{|z|^{a}}$.

To deal with the concentration phenomena, we will need a variant of the last theorem.

Theorem A.3. Let $\left(u^{\varepsilon}\right)_{\varepsilon>0} \in C^{2}(\mathbb{D} \backslash\{0\})$ solve

$$
\Delta u^{\varepsilon}(z)=\chi^{a} f^{\varepsilon}(z) \text { in } \mathbb{D},
$$

with $f^{\varepsilon} \in L^{p}(\mathbb{D})$ for $2 \leq p \leq \infty, a \in \mathbb{R}$ and $\chi:=\sqrt{\varepsilon^{2}+r^{2}}$. Then

$$
u_{z}^{\varepsilon}(z)=P^{\varepsilon}(z)+\chi^{a} T^{\varepsilon}(z)
$$

with $P^{\varepsilon} \in \mathbb{C}_{\lceil a\rceil}[X]$ and $T^{\varepsilon}=O\left(\chi^{1-\frac{2}{p}-\kappa}\right)$ for all $\kappa>0$. Here $\lceil a\rceil$ is the upper integral part of $a$. More precisely one has

$$
\left\|\frac{T^{\varepsilon}}{\chi^{1-\frac{2}{p}-\kappa}}\right\|_{L^{\infty}(\mathbb{D})} \leq C_{\kappa}\left(\left\|f^{\varepsilon}\right\|_{L^{p}(\mathbb{D})}+\left\|u^{\varepsilon}\right\|_{C^{1}(\partial \mathbb{D})}\right) .
$$

Additionally:

$$
u_{z z}^{\varepsilon}(z)=P_{z}^{\varepsilon}+\chi^{a} Q^{\varepsilon}
$$

with $Q^{\varepsilon} \in L^{p^{\prime}}(\mathbb{D})$ for all $p^{\prime}<p$ and

$$
\left\|Q^{\varepsilon}\right\|_{L^{p^{\prime}(\mathbb{D})}} \leq C_{p^{\prime}}\left(\left\|f^{\varepsilon}\right\|_{L^{p}(\mathbb{D})}+\left\|u^{\varepsilon}\right\|_{C^{1}(\partial \mathbb{D})}\right)
$$

In fact $Q^{\varepsilon}=\frac{\left(\chi^{a} T^{\varepsilon}(z)\right)_{z}}{\chi^{a}}$.

Proof. We first give a small lemma which can help one understand the concrete impact of $\chi$ : 
Lemma A.4. For all $a, b \in \mathbb{R}_{+}$, there exists a constant $C_{a, b}$ such that

$$
\frac{\varepsilon^{a} r^{b}}{\chi^{a+b}} \leq C_{a, b}
$$

Similarly for all $a \in \mathbb{R}_{+}$, there exists $C_{a} \in \mathbb{R}_{+}^{*}$ such that

$$
\frac{1}{C_{a}} \leq \frac{\varepsilon^{a}+r^{a}}{\chi^{a}} \leq C_{a}
$$

Here $C_{a}$ (respectively $C_{a, b}$ ) depends solely on $a$ (and $b$ ), and not on $\varepsilon$ or $r$.

We then write

$$
\Delta u^{\varepsilon}=\left(\varepsilon^{a}+r^{a}\right) \frac{\chi^{a}}{\varepsilon^{a}+r^{a}} f^{\varepsilon}=\left(\varepsilon^{a}+r^{a}\right) \tilde{f}^{\varepsilon},
$$

where $\tilde{f}^{\varepsilon}=\frac{\chi^{a}}{\varepsilon^{a}+r^{a}} f^{\varepsilon}$ satisfies, thanks to 154 ,

$$
\left\|\widetilde{f}^{\varepsilon}\right\|_{L^{p}(\mathbb{D})} \leq C_{a}\left\|f^{\varepsilon}\right\|_{L^{p}(\mathbb{D})}
$$

We can then use Green's formula to write

$$
\begin{aligned}
u_{z}^{\varepsilon}(z) & =\frac{1}{2 \pi} \int_{\partial \mathbb{D}}\left(\frac{\bar{z}-\bar{x}}{|z-x|^{2}} \partial_{\nu} u^{\varepsilon}(x)-u^{\varepsilon}(x) \partial \frac{\bar{z}-\bar{x}}{|z-x|^{2}}\right) d \sigma(x) \\
- & \frac{1}{2 \pi} \int_{\mathbb{D}} \frac{\bar{z}-\bar{x}}{|z-x|^{2}}\left(\varepsilon^{a}+r^{a}\right) \widetilde{f}^{\varepsilon}(x) d x \\
& =\frac{1}{2 \pi} \int_{\partial \mathbb{D}}\left(\frac{\bar{z}-\bar{x}}{|z-x|^{2}} \partial_{\nu} u^{\varepsilon}(x)-u^{\varepsilon}(x) \partial \frac{\bar{z}-\bar{x}}{|z-x|^{2}}\right) d \sigma(x) \\
& -\frac{1}{2 \pi} \int_{\mathbb{D}} \frac{\bar{z}-\bar{x}}{|z-x|^{2}} \varepsilon^{a} \widetilde{f}^{\varepsilon}(x) d x-\frac{1}{2 \pi} \int_{\mathbb{D}} \frac{\bar{z}-\bar{x}}{|z-x|^{2}} r^{a} \widetilde{f}^{\varepsilon}(x) d x \\
& =I_{0}^{\varepsilon}(z)+I_{1}^{\varepsilon}(z)+I_{2}^{\varepsilon}(z) .
\end{aligned}
$$

We can then successively estimate the three terms as in the proof of theorem A.2 and write

$$
I_{0}^{\varepsilon}(z)=P_{0}^{\varepsilon}(z)+z^{\lceil a\rceil+1} T_{0}^{\varepsilon},
$$

where $P_{0}^{\varepsilon}$ is a polynomial of degree at most $\lceil a\rceil$ and whose coefficients are bounded by $\left\|u^{\varepsilon}\right\|_{C^{1}(\partial \mathbb{D})}$, and

$$
\left\|T_{0}^{\varepsilon}\right\|_{L^{\infty}(\mathbb{D})} \leq C\left\|u^{\varepsilon}\right\|_{C^{1}(\mathbb{D})} .
$$

Working as in 3 we write

$$
\begin{aligned}
& I_{1}^{\varepsilon}(z)=C^{\varepsilon}+\varepsilon^{a} T_{1}^{\varepsilon}(z) \\
& I_{2}^{\varepsilon}(z)=P_{2}^{\varepsilon}+r^{a} T_{2}^{\varepsilon}(z)
\end{aligned}
$$

where $C^{\varepsilon}$ is a constant and $P_{2}^{\varepsilon}$ a polynomial of degree at most $\lceil a\rceil$, both bounded by $\left\|u^{\varepsilon}\right\|_{C^{1}(\partial \mathbb{D})}$, while

$$
\begin{aligned}
& \left\|\frac{T_{1}^{\varepsilon}}{r^{1-\frac{2}{p}-\kappa}}\right\|_{L^{\infty}(\mathbb{D})} \leq C_{\kappa}\left(\left\|\tilde{f}^{\varepsilon}\right\|_{L^{p}(\mathbb{D})}+\left\|u^{\varepsilon}\right\|_{C^{1}(\mathbb{D})}\right) \\
& \left\|\frac{T_{2}^{\varepsilon}}{r^{1-\frac{2}{p}-\kappa}}\right\|_{L^{\infty}(\mathbb{D})} \leq C_{\kappa}\left(\left\|\tilde{f}^{\varepsilon}\right\|_{L^{p}(\mathbb{D})}+\left\|u^{\varepsilon}\right\|_{C^{1}(\mathbb{D})}\right) .
\end{aligned}
$$

In the end, combining (157) and 158 yields

$$
u_{z}^{\varepsilon}=P^{\varepsilon}+\varepsilon^{a} T_{1}^{\varepsilon}+r^{a} T_{3}^{\varepsilon},
$$

where $P^{\varepsilon}$ is a polynomial of degree at most $\lceil a\rceil, T_{1}^{\varepsilon}$ is as previously stated and $T_{3}^{\varepsilon}$ still satisfies

$$
\left\|\frac{T_{3}^{\varepsilon}}{r^{1-\frac{2}{p}-\kappa}}\right\|_{L^{\infty}(\mathbb{D})} \leq C_{\kappa}\left(\left\|\tilde{f}^{\varepsilon}\right\|_{L^{p}(\mathbb{D})}+\left\|u^{\varepsilon}\right\|_{C^{1}(\mathbb{D})}\right) .
$$


Proceeding similarly then ensures that

$$
u_{z z}^{\varepsilon}=P_{z}^{\varepsilon}+\varepsilon^{a} Q_{1}^{\varepsilon}+r^{a} Q_{2}^{\varepsilon},
$$

where $Q_{1}^{\varepsilon}=T_{1, z}^{\varepsilon}$ and $Q_{2}^{\varepsilon}=\frac{\left(r^{a} T_{3}^{\varepsilon}\right)_{z}}{r^{a}}$ satisfy for all $p^{\prime}<p$

$$
\begin{aligned}
& \left\|Q_{1}^{\varepsilon}\right\|_{L^{p^{\prime}}(\mathbb{D})} \leq C_{p^{\prime}}\left(\left\|\widetilde{f}^{\varepsilon}\right\|_{L^{p}(\mathbb{D})}+\left\|u^{\varepsilon}\right\|_{C^{1}(\partial \mathbb{D})}\right), \\
& \left\|Q_{3}^{\varepsilon}\right\|_{L^{p^{\prime}}(\mathbb{D})} \leq C_{p^{\prime}}\left(\left\|\widetilde{f}^{\varepsilon}\right\|_{L^{p}(\mathbb{D})}+\left\|u^{\varepsilon}\right\|_{C^{1}(\partial \mathbb{D})}\right) .
\end{aligned}
$$

Let us notice that the estimate on $Q_{1}^{\varepsilon}$ is not stricto sensu derived from the proof of theorem A.2 but from similar classical Calderon-Zygmund estimates.

From (159) we write $u_{z}^{\varepsilon}=P^{\varepsilon}+\chi^{a} T^{\varepsilon}$ with

$$
T^{\varepsilon}=\frac{\varepsilon^{a}}{\chi^{a}} T_{1}^{\varepsilon}+\frac{r^{a}}{\chi^{a}} T_{3}^{\varepsilon}
$$

which then satisfies

$$
\begin{aligned}
\left\|\frac{T^{\varepsilon}}{\chi^{1-\frac{2}{p}-\kappa}}\right\|_{L^{\infty}(\mathbb{D})} & \leq\left\|\frac{\varepsilon^{a}}{\chi^{a}}\right\|_{L^{\infty}(\mathbb{D})}\left\|\frac{T_{1}^{\varepsilon}}{\chi^{1-\frac{2}{p}-\kappa}}\right\|_{L^{\infty}(\mathbb{D})}+\left\|\frac{r^{a}}{\chi^{a}}\right\|_{L^{\infty}(\mathbb{D})}\left\|\frac{T_{3}^{\varepsilon}}{\chi^{1-\frac{2}{p}-\kappa}}\right\|_{L^{\infty}(\mathbb{D})} \\
& \leq\left\|\frac{\varepsilon^{a}}{\chi^{a}}\right\|_{L^{\infty}(\mathbb{D})}\left\|\frac{T_{1}^{\varepsilon}}{r^{1-\frac{2}{p}-\kappa}}\right\|_{L^{\infty}(\mathbb{D})}\left\|\frac{r^{1-\frac{2}{p}-\kappa}}{\chi^{1-\frac{2}{p}-\kappa}}\right\|_{L^{\infty}(\mathbb{D})} \\
& +\left\|\frac{r^{a}}{\chi^{a}}\right\|_{L^{\infty}(\mathbb{D})}\left\|\frac{T_{3}^{\varepsilon}}{r^{1-\frac{2}{p}-\kappa}}\right\|_{L^{\infty}(\mathbb{D})}\left\|\frac{r^{1-\frac{2}{p}-\kappa}}{\chi^{1-\frac{2}{p}-\kappa}}\right\|_{L^{\infty}(\mathbb{D})} \\
& \leq C_{\kappa}\left(\left\|\tilde{f}^{\varepsilon}\right\|_{L^{p}(\mathbb{D})}+\left\|u^{\varepsilon}\right\|_{C^{1}(\mathbb{D})}\right),
\end{aligned}
$$

using lemma A.4.

From 160 we write $u_{z z}^{\varepsilon}=P_{z}^{\varepsilon}+\chi^{a} Q^{\varepsilon}$ with

$$
Q^{\varepsilon}=\frac{\varepsilon^{a}}{\chi^{a}} Q_{1}^{\varepsilon}+\frac{r^{a}}{\chi^{a}} Q_{3}^{\varepsilon}=\frac{\left(\chi^{a} T^{\varepsilon}\right)_{z}}{\chi^{a}}
$$

which then satisfies

$$
\begin{aligned}
\left\|Q^{\varepsilon}\right\|_{L^{p^{\prime}(\mathbb{D})}} & \leq\left\|\frac{\varepsilon^{a}}{\chi^{a}}\right\|_{L^{\infty}(\mathbb{D})}\left\|Q_{1}^{\varepsilon}\right\|_{L^{p^{\prime}(\mathbb{D})}}+\left\|\frac{r^{a}}{\chi^{a}}\right\|_{L^{\infty}(\mathbb{D})}\left\|Q_{3}^{\varepsilon}\right\|_{L^{p^{\prime}(\mathbb{D})}} \\
& \leq C_{p^{\prime}}\left(\left\|\tilde{f}^{\varepsilon}\right\|_{L^{p}(\mathbb{D})}+\left\|u^{\varepsilon}\right\|_{C^{1}(\mathbb{D})}\right),
\end{aligned}
$$

using lemma A.4

Remark A.4.1. We must point out that the expansion given by theorem A.3 is by no means unique. Indeed if for instance $u_{z}=P^{\varepsilon}+\chi^{m} T^{\varepsilon}$, then one could readily write

$$
u_{z}=P^{\varepsilon}+\varepsilon^{m+1}+\chi^{m}\left(T^{\varepsilon}+\frac{\varepsilon^{m+1}}{\chi^{m}}\right)
$$

with $T^{\varepsilon}+\frac{\varepsilon^{m+1}}{\chi^{m}}$ still satisfying 161 .

Theorem A.3 can be applied several times to prove an increased regularity on the higher order terms:

Lemma A.5. Let $u^{\varepsilon} \in C^{2}(\mathbb{D} \backslash\{0\})$ such that

$$
\Delta u^{\varepsilon}=\chi^{a} f^{\varepsilon},
$$

with $f^{\varepsilon} \in L^{\infty}$ and

$$
\Delta\left(\nabla u^{\varepsilon}\right)=\chi^{a-1} g^{\varepsilon},
$$


with $g^{\varepsilon} \in L^{p}$. Then

$$
u_{z}^{\varepsilon}=P^{\varepsilon}+\mu^{\varepsilon}
$$

where $P^{\varepsilon}$ is a complex polynomial of degree at most $\lceil a\rceil$, and $\mu^{\varepsilon}$ such that

$$
\frac{\left|\mu^{\varepsilon}\right|}{\chi^{a+1-\kappa}}+\frac{\left|\nabla \mu^{\varepsilon}\right|}{\chi^{a-\frac{2}{p}-\kappa}} \leq C_{\kappa}\left(\left\|f^{\varepsilon}\right\|_{L^{\infty}(\mathbb{D})}+\left\|g^{\varepsilon}\right\|_{L^{p}(\mathbb{D})}+\left\|u^{\varepsilon}\right\|_{C^{2}(\partial \mathbb{D})}\right)
$$

and

$$
\left\|\frac{\nabla^{2} \mu^{\varepsilon}}{\chi^{a-1}}\right\|_{L^{p^{\prime}(\mathbb{D})}} \leq C_{p^{\prime}}\left(\left\|f^{\varepsilon}\right\|_{L^{\infty}(\mathbb{D})}+\left\|g^{\varepsilon}\right\|_{L^{p}(\mathbb{D})}+\left\|u^{\varepsilon}\right\|_{C^{2}(\partial \mathbb{D})}\right) .
$$

Proof. We apply theorem A.3 twice and decompose $u_{z}$ and $\left(u_{z}\right)_{z}$ :

$$
\begin{aligned}
u_{z}^{\varepsilon} & =P_{1}^{\varepsilon}+\mu_{1}^{\varepsilon} \\
\left(u_{z}^{\varepsilon}\right)_{z} & =P_{2}^{\varepsilon}+\mu_{2}^{\varepsilon}
\end{aligned}
$$

where

$$
\begin{aligned}
& \frac{\left|\mu_{1}^{\varepsilon}\right|}{\chi^{a+1-\kappa}}+\frac{\left|\mu_{2}^{\varepsilon}\right|}{\chi^{a-\frac{2}{p}-\kappa}} \leq C_{\kappa}\left(\left\|f^{\varepsilon}\right\|_{L^{\infty}(\mathbb{D})}+\left\|g^{\varepsilon}\right\|_{L^{p}(\mathbb{D})}+\left\|u^{\varepsilon}\right\|_{C^{2}(\partial \mathbb{D})}\right) \\
&\left\|\frac{\nabla \mu_{1}^{\varepsilon}}{\chi^{a}}\right\|_{L^{p_{1}^{\prime}(\mathbb{D})}}+\left\|\frac{\nabla \mu_{2}^{\varepsilon}}{\chi^{a-1}}\right\|_{L^{p_{2}^{\prime}(\mathbb{D})}} \leq C_{p_{1}^{\prime}, p_{2}^{\prime}}\left(\left\|f^{\varepsilon}\right\|_{L^{\infty}(\mathbb{D})}+\left\|g^{\varepsilon}\right\|_{L^{p}(\mathbb{D})}+\left\|u^{\varepsilon}\right\|_{C^{2}(\partial \mathbb{D})}\right),
\end{aligned}
$$

for all $p_{1}^{\prime}<\infty$ and $p_{2}^{\prime}<p$. We then enjoy two expressions for $u_{z z}$ :

$$
u_{z z}=P_{1, z}^{\varepsilon}+\mu_{1, z}^{\varepsilon}=P_{2}^{\varepsilon}+\mu_{2}^{\varepsilon}
$$

Consequently

$$
P_{1, z}^{\varepsilon}-P_{2}^{\varepsilon}=\mu_{2}^{\varepsilon}-\mu_{1, z}^{\varepsilon},
$$

which in turn, combined with 164, implies that

$$
\int_{\mathbb{D}}\left|\frac{P_{1, z}^{\varepsilon}-P_{2}^{\varepsilon}}{\chi^{a}}\right|^{s} d z \leq C_{s}\left(\left\|f^{\varepsilon}\right\|_{L^{\infty}(\mathbb{D})}+\left\|g^{\varepsilon}\right\|_{L^{p}(\mathbb{D})}+\left\|u^{\varepsilon}\right\|_{C^{2}(\partial \mathbb{D})}\right),
$$

for all $s<\infty$. We decompose

$$
P_{1, z}^{\varepsilon}-P_{2}^{\varepsilon}=\sum_{q=0}^{\lfloor a\rfloor} p_{q}^{\varepsilon} z^{q}
$$

and can state for a given $R_{0}>0$

$$
\int_{\mathbb{D}_{\epsilon R_{0}}}\left|\frac{\sum_{q=0}^{\lfloor a\rfloor} p_{q}^{\varepsilon} z^{q}}{\chi^{a}}\right|^{s} d z \leq \int_{\mathbb{D}}\left|\frac{\sum_{q=0}^{\lfloor a\rfloor} p_{q}^{\varepsilon} z^{q}}{\chi^{a}}\right|^{p} \leq C_{s}\left(\left\|f^{\varepsilon}\right\|_{L^{\infty}(\mathbb{D})}+\left\|g^{\varepsilon}\right\|_{L^{p}(\mathbb{D})}+\left\|u^{\varepsilon}\right\|_{C^{2}(\partial \mathbb{D})}\right) .
$$

Changing variables yields

$$
\int_{\mathbb{D}_{R_{0}}}\left|\frac{\sum_{q=0}^{\lfloor a\rfloor} \frac{p_{q}^{\varepsilon}}{\varepsilon^{a-q-\frac{2}{p}}} z^{q}}{\sqrt{1+r^{2}}}\right|^{s} d z \leq C_{s}\left(\left\|f^{\varepsilon}\right\|_{L^{\infty}(\mathbb{D})}+\left\|g^{\varepsilon}\right\|_{L^{p}(\mathbb{D})}+\left\|u^{\varepsilon}\right\|_{C^{2}(\partial \mathbb{D})}\right) .
$$

And since on $\mathbb{D}_{\mathbb{R}_{0}}, \frac{1}{1+r^{2}} \geq \frac{1}{1+R_{0}^{2}}$, we deduce

$$
\int_{\mathbb{D}_{R_{0}}}\left|\sum_{q=0}^{\lfloor a\rfloor} \frac{p_{q}^{\varepsilon}}{\varepsilon^{a-q-\frac{2}{p}}} z^{q}\right|^{s} d z \leq C_{s, R_{0}}\left(\left\|f^{\varepsilon}\right\|_{L^{\infty}(\mathbb{D})}+\left\|g^{\varepsilon}\right\|_{L^{p}(\mathbb{D})}+\left\|u^{\varepsilon}\right\|_{C^{2}(\partial \mathbb{D})}\right) .
$$


It is now important to notice that the left-hand term in 165 is in fact a polynomial in $R_{0}$, which is uniformly bounded in $\varepsilon$ on compacts of $\mathbb{C}$. All its coefficients are thus uniformly bounded in $\varepsilon$, and straightforward computations then yield:

$$
\forall s<\infty \quad \forall j \leq\lfloor a\rfloor \quad \forall \varepsilon>0 \quad\left|\frac{p_{q}^{\varepsilon}}{\varepsilon^{a-q-\frac{2}{s}}}\right| \leq C_{s}\left(\left\|f^{\varepsilon}\right\|_{L^{\infty}(\mathbb{D})}+\left\|g^{\varepsilon}\right\|_{L^{p}(\mathbb{D})}+\left\|u^{\varepsilon}\right\|_{C^{2}(\partial \mathbb{D})}\right)
$$

which thanks to lemma A.4 translates on $P_{1, z}^{\varepsilon}-P_{2}^{\varepsilon}$ as

$$
\forall s<\infty \quad\left|\frac{P_{1, z}^{\varepsilon}-P_{2}^{\varepsilon}}{\chi^{a-\frac{2}{s}}}\right| \leq C_{s}\left(\left\|f^{\varepsilon}\right\|_{L^{\infty}(\mathbb{D})}+\left\|g^{\varepsilon}\right\|_{L^{p}(\mathbb{D})}+\left\|u^{\varepsilon}\right\|_{C^{2}(\partial \mathbb{D})}\right)
$$

and

$$
\forall s<\infty \quad\left|\frac{\left(P_{1, z}^{\varepsilon}-P_{2}^{\varepsilon}\right)_{z}}{\chi^{a-\frac{2}{s}-1}}\right| \leq C_{s}\left(\left\|f^{\varepsilon}\right\|_{L^{\infty}(\mathbb{D})}+\left\|g^{\varepsilon}\right\|_{L^{p}(\mathbb{D})}+\left\|u^{\varepsilon}\right\|_{C^{2}(\partial \mathbb{D})}\right) .
$$

Now since $\mu_{1, z}^{\varepsilon}=\mu_{2}^{\varepsilon}-\left(P_{1, z}^{\varepsilon}-P_{2}^{\varepsilon}\right)$ we can combine 164 and (166) to find for all $\kappa>0$

$$
\left|\frac{\mu_{1, z}^{\varepsilon}}{\chi^{a-\frac{2}{p}-\kappa}}\right| \leq C_{\kappa}\left(\left\|f^{\varepsilon}\right\|_{L^{\infty}(\mathbb{D})}+\left\|g^{\varepsilon}\right\|_{L^{p}(\mathbb{D})}+\left\|u^{\varepsilon}\right\|_{C^{2}(\partial \mathbb{D})}\right) .
$$

In addition, since $\mu_{1, z z}^{\varepsilon}=\mu_{2, z}^{\varepsilon}-\left(P_{1, z}^{\varepsilon}-P_{2}^{\varepsilon}\right)_{z}$, 164) and (167) yield for all $p^{\prime}<p$ :

$$
\left\|\frac{\mu_{1, z z}^{\varepsilon}}{\chi^{a-1}}\right\|_{L^{p^{\prime}(\mathbb{D})}} \leq C_{p^{\prime}}\left(\left\|f^{\varepsilon}\right\|_{L^{\infty}(\mathbb{D})}+\left\|g^{\varepsilon}\right\|_{L^{p}(\mathbb{D})}+\left\|u^{\varepsilon}\right\|_{C^{2}(\partial \mathbb{D})}\right) .
$$

Applying similarly theorem A.3 to $u_{\bar{z}}^{\varepsilon}$ yields controls akin to 168$)$ and $(169)$ on the missing terms in the gradient and the Hessian, which concludes the proof.

A cautious reader might have noticed that we have in fact proved the following lemma:

Lemma A.6. Let $u \in \mathbb{N}, v \geq u$ and $P^{\varepsilon}=\sum_{j=0}^{u} p_{j}^{\varepsilon} z^{j} \in \mathbb{C}_{u}[X]$ such that

$$
\forall p<\infty \quad \frac{P^{\varepsilon}}{\chi^{v}} \in L^{p} .
$$

Then

$$
\forall \nu>0 \quad \forall j \leq u \quad\left|\frac{p_{j}^{\varepsilon}}{\varepsilon^{v-j-\nu}}\right| \leq C_{\nu}
$$

We will also use a corresponding result for polynomials in $z$ and $\bar{z}$ :

Lemma A.7. Let $u \in \mathbb{N}, v \geq u$ and $P^{\varepsilon}=\sum_{i+j=0}^{u} p_{i, j}^{\varepsilon} z^{i} \bar{z}^{j}$ such that

$$
\forall p<\infty \quad \frac{P^{\varepsilon}}{\chi^{v}} \leq C .
$$

Then

$$
\forall \nu>0 \quad \forall i+j \leq u \quad\left|\frac{p_{i, j}^{\varepsilon}}{\varepsilon^{v-i-j}}\right| \leq C_{\nu} .
$$

Applying lemma A.5 several times yields:

Corollary A.8. Let $u^{\varepsilon} \in C^{2}(\mathbb{D} \backslash\{0\})$ such that, for $a \geq t$

$$
\begin{aligned}
\Delta u^{\varepsilon} & =\chi^{a} f_{0}^{\varepsilon}, \\
\Delta \nabla u^{\varepsilon} & =\chi^{a-1} f_{1}^{\varepsilon} \\
\cdots & \\
\Delta \nabla^{t} u^{\varepsilon} & =\chi^{a-t} f_{t}^{\varepsilon}
\end{aligned}
$$


with $f_{j}^{\varepsilon} \in L^{\infty}(\mathbb{D})$ for $j \leq t-1$ and $f_{t}^{\varepsilon} \in L^{p}(\mathbb{D})$. Then

$$
u_{z}^{\varepsilon}=P^{\varepsilon}+\mu^{\varepsilon},
$$

where $P^{\varepsilon}$ is a complex polynomial of degree at most $\lceil a\rceil$, and $\mu^{\varepsilon}$ such that

$$
\frac{\left|\mu^{\varepsilon}\right|}{\chi^{a+1-\kappa}}+\frac{\left|\nabla \mu^{\varepsilon}\right|}{\chi^{a-\kappa}}+\cdots+\frac{\left|\nabla^{t} \mu^{\varepsilon}\right|}{\chi^{a+1-t-\frac{2}{p}-\kappa}} \leq C_{\kappa}\left(\sum_{q=0}^{t}\left\|f_{q}^{\varepsilon}\right\|_{L^{\infty}(\mathbb{D})}+\left\|u^{\varepsilon}\right\|_{C^{t+1}(\partial \mathbb{D})}\right)
$$

and

$$
\left\|\frac{\nabla^{t+1} \mu^{\varepsilon}}{\chi^{a-t}}\right\|_{L^{p^{\prime}(\mathbb{D})}} \leq C_{p^{\prime}}\left(\sum_{q=0}^{t}\left\|f_{q}^{\varepsilon}\right\|_{L^{\infty}(\mathbb{D})}+\left\|u^{\varepsilon}\right\|_{C^{t+1}(\partial \mathbb{D})}\right) .
$$

Proof. The proof is a recurrence whose initialization is theorem A.3 and whose heredity is obtained by applying lemma is A.5 to $\nabla^{s} u^{\varepsilon}$.

\section{A.2 Auxiliary formulas}

In the following, given $\vec{\Phi}$ a Willmore conformal immersion, and $\vec{L}, S, \vec{R}$ defined in 34 , we wish to prove:

$$
\vec{R}_{z}=2(H+i V) \vec{\Phi}_{z}-i S_{z} \vec{n}
$$

where $V=\frac{1}{2}\langle\vec{L}, \vec{n}\rangle$. Indeed, since

$$
S_{z}=\left\langle\vec{L}, \vec{\Phi}_{z}\right\rangle \text { and } \vec{R}_{z}=\vec{L} \times \vec{\Phi}_{z}+2 H \vec{\Phi}_{z}
$$

we successively compute:

$$
\left\langle\vec{R}_{z}, \vec{\Phi}_{z}\right\rangle=0,\left\langle\vec{R}_{z}, \vec{\Phi}_{\bar{z}}\right\rangle=\frac{e^{2 \lambda}}{2}(2 H+i\langle\vec{L}, \vec{n}\rangle) \text { and }\left\langle\vec{R}_{z}, \vec{n}\right\rangle=-i S_{z},
$$

which proves the desired equality.

\section{A.3 Curvature formulas for branched immersions}

We first give a version of Gauss-Bonnet formula taking branch points and branched ends into account. We refer the reader to theorem 2.6 in [10.

Proposition A.9. Let $\Sigma$ be a compact Riemann surface and $\vec{\Phi}: \Sigma \rightarrow \mathbb{R}^{3} \cup\{\infty\}$ be a branched immersion with a finite number of ends. Let $p_{1}, \ldots, p_{n}$ be its branch points of respective orders $a_{1}+1, \ldots, a_{n}+1$ and $q_{1}, \ldots, q_{m}$ its ends of respective orders $b_{1}-1, \ldots b_{m}-1$. We denote $\chi(\Sigma)$ the Euler characteristic of $\Sigma, g$ the metric induced on $\Sigma$ by $\vec{\Phi}$ and $K$ its Gauss curvature. Hence

$$
\int_{\Sigma} K d \operatorname{vol}_{g}=2 \pi\left(\chi(\Sigma)+\sum_{i=1}^{n} a_{i}-\sum_{j=1}^{m} b_{j}\right) .
$$

Following are a few useful formulas linking the different notions of curvature. The computations are done in appendix A.1 of [19] .

Proposition A.10. Let $\Sigma$ be a compact Riemann surface and $\vec{\Phi}: \Sigma \rightarrow \mathbb{R}^{3} \cup\{\infty\}$ be a branched immersion with a finite number of ends. Let $g$ be the induced metric, $\vec{n}$ be its Gauss map, $H$ its mean curvature, $\AA$ its tracefree second fundamental form and $K$ its Gauss curvature. Then

$$
\int_{\Sigma}|\nabla \vec{n}|^{2} d \operatorname{vol}_{g}=4 \int_{\Sigma} H^{2} d \operatorname{vol}_{g}-2 \int_{\Sigma} K d \operatorname{vol}_{g}=2 \int_{\Sigma}|\AA|^{2} d \operatorname{vol}_{g}+2 \int_{\Sigma} K d \operatorname{vol}_{g} .
$$




\section{References}

[1] T. Apostol. Modular functions and Dirichlet series in number theory, volume 41 of Graduate Texts in Mathematics. Springer-Verlag, New York, second edition, 1990.

[2] Y. Bernard. Noether's theorem and the Willmore functional. Adv. Calc. Var., 9(3):217-234, 2016.

[3] Y. Bernard and T. Rivière. Singularity removability at branch points for Willmore surfaces. Pacific J. Math., 265(2):257-311, 2013.

[4] Y. Bernard and T. Rivière. Energy quantization for Willmore surfaces and applications. Ann. of Math. (2), 180(1):87-136, 2014.

[5] W. Blaschke. Vorlesungen über Integralgeometrie. Deutscher Verlag der Wissenschaften, Berlin, 1955. 3te Aufl.

[6] H. Brezis and J.-M. Coron. Convergence of solutions of $H$-systems or how to blow bubbles. Arch. Ration. Mech. Anal., 89(1):21-56, 1985.

[7] R. Bryant. A duality theorem for Willmore surfaces. J. Differential Geom., 20(1):23-53, 1984.

[8] C. Chen and F. Gackstatter. Elliptische und hyperelliptische Funktionen und vollständige Minimalflächen vom Enneperschen Typ. Math. Ann., 259(3):359-369, 1982.

[9] E. Kuwert and R. Schätzle. The Willmore flow with small initial energy. J. Differential Geom., 57(3):409$441,2001$.

[10] T. Lamm and H. Nguyen. Branched Willmore spheres. J. Reine Angew. Math., 701:169-194, 2015.

[11] P. Laurain. Asymptotic analysis for surfaces with large constant mean curvature and free boundaries. Ann. Inst. H. Poincaré Anal. Non Linéaire, 29(1):109-129, 2012.

[12] P. Laurain and T. Rivière. Energy quantization of Willmore surfaces at the boundary of the moduli space. Duke Math. J., 167(11):2073-2124, 2018.

[13] P. Laurain and T. Rivière. Optimal estimate for the gradient of Green's function on degenerating surfaces and applications. Comm. Anal. Geom., 26(4):887-913, 2018.

[14] P. Li and S. Yau. A new conformal invariant and its applications to the Willmore conjecture and the first eigenvalue of compact surfaces. Invent. Math., 69(2):269-291, 1982.

[15] Y. Li. Some remarks on Willmore surfaces embedded in $\mathbb{R}^{3}$. J. Geom. Anal., 26(3):2411-2424, 2016.

[16] F. López. The classification of complete minimal surfaces with total curvature greater than $-12 \pi$. Trans. Amer. Math. Soc., 334(1):49-74, 1992.

[17] F. López and F. Martín. Complete minimal surfaces in $\mathbf{R}^{3}$. Publ. Mat., 43(2):341-449, 1999.

[18] N. Marque. Conformal gauss map geometry and application to Willmore surfaces in model spaces. arXiv:1903.07475, 2019.

[19] N. Marque. An $\varepsilon$-regularity result with mean curvature control for Willmore immersions and application to minimal bubbling. arXiv:1904.05215, 2019.

[20] A. Michelat and T. Rivière. The classification of branched Willmore spheres in the 3 -sphere and the 4-sphere. arXiv:1706.01405, 2017.

[21] A. Michelat and T. Rivière. Private communications. 2019.

[22] Robert Osserman. A survey of minimal surfaces. Dover Publications, Inc., New York, second edition, 1986.

[23] U. Pinkall. Hopf tori in $S^{3}$. Invent. Math., 81(2):379-386, 1985.

[24] T. Rivière. Analysis aspects of Willmore surfaces. Invent. Math., 174(1):1-45, 2008.

[25] T. J. Willmore. Riemannian geometry. Oxford Science Publications. The Clarendon Press, Oxford University Press, New York, 1993. 